\title{
MMCTP: \\ A radiotherapy research environment for Monte Carlo and patient-specific treatment planning
}

\author{
Andrew William Alexander
}

Master of Science

Medical Physics

McGill University

Montreal,Quebec

December 2006

A thesis submitted to the Faculty of Graduate Studies and Research of McGill University in partial fulfillment of the requirements of the degree of Master of Science

(C) Andrew William Alexander 2006 


$\begin{array}{ll}\begin{array}{l}\text { Library and } \\ \text { Archives Canada }\end{array} & \begin{array}{l}\text { Bibliothèque et } \\ \text { Archives Canada }\end{array} \\ \begin{array}{l}\text { Published Heritage } \\ \text { Branch }\end{array} & \begin{array}{l}\text { Direction du } \\ \text { Patrimoine de l'édition }\end{array} \\ \begin{array}{l}\text { 395 Wellington Street } \\ \text { Ottawa ON K1A 0N4 }\end{array} & \begin{array}{l}\text { 395, rue Wellington } \\ \text { Ottawa ON K1A ON4 } \\ \text { Canada }\end{array} \\ \end{array}$

Your file Votre référence ISBN: 978-0-494-32812-5 Our file Notre référence ISBN: 978-0-494-32812-5

NOTICE:

The author has granted a nonexclusive license allowing Library and Archives Canada to reproduce, publish, archive, preserve, conserve, communicate to the public by telecommunication or on the Internet, loan, distribute and sell theses worldwide, for commercial or noncommercial purposes, in microform, paper, electronic and/or any other formats.

The author retains copyright ownership and moral rights in this thesis. Neither the thesis nor substantial extracts from it may be printed or otherwise reproduced without the author's permission.
AVIS:

L'auteur a accordé une licence non exclusive permettant à la Bibliothèque et Archives Canada de reproduire, publier, archiver, sauvegarder, conserver, transmettre au public par télécommunication ou par l'Internet, prêter, distribuer et vendre des thèses partout dans le monde, à des fins commerciales ou autres, sur support microforme, papier, électronique et/ou autres formats.

L'auteur conserve la propriété du droit d'auteur et des droits moraux qui protège cette thèse. $\mathrm{Ni}$ la thèse ni des extraits substantiels de celle-ci ne doivent être imprimés ou autrement reproduits sans son autorisation.
In compliance with the Canadian

Privacy Act some supporting forms may have been removed from this thesis.

While these forms may be included in the document page count, their removal does not represent any loss of content from the thesis.
Conformément à la loi canadienne sur la protection de la vie privée, quelques formulaires secondaires ont été enlevés de cette thèse.

Bien que ces formulaires aient inclus dans la pagination, il n'y aura aucun contenu manquant. 


\section{ACKNOWLEDGEMENTS}

I would like to thank my two supervisors, Dr. Jan Seuntjens and Dr. François DeBlois for their guidance and enthusiastic encouragement for this work. I have enjoyed my time while working on this project and gained a wealth of information in the process. The success of this project is largely due to their support.

I would like to thank Dr. Ervin B. Podgoršak for providing me the opportunity to join the McGill Medical Physics program and his encouraging words throughout my studies.

I would also like to express my gratitude to the staff of the McGill Medical Physics Unit. The infrastructure and research tools available to me are due to the timeless efforts of various people throughout the department.

Support from the senior students was truly appreciated. In particular: Khalid AlYahya for his expertise in Monte Carlo applications, Gabriela Stroian for her expertise in Monte Carlo patient recalculations, and Geneviève Jarry for her expertise in measurement analysis.

Funding in support of the research presented in this thesis has been provided by CIHR grant MOP 79448 and NCIC TFF grant \# 016298. 


\begin{abstract}
Accurate dose calculations and analysis tools are essential to radiotherapy treatment planning. Radiotherapy deliveries utilize the information provided by the treatment planning system and it is generally accepted that clinical outcome can be improved if accuracy in the dose delivery is further improved. Proven Monte Carlo calculations increase the planning accuracy however, most radiotherapy departments do not use Monte Carlo. The McGill Monte Carlo treatment planning system, MMCTP, provides a flexible software environment to integrate Monte Carlo planning with current and new treatment modalities and deliveries. The MMCTP design consists of a graphical user interface, which runs on a simple workstation connected through standard secure-shell protocol to a cluster for lengthy Monte Carlo calculations. The impact of this tool lies in the fact that it allows for systematic, platform independent, large-scale Monte Carlo planning calculations for different treatment sites. Various measurements and patient recalculations were preformed to validate the software and ensure proper functionality.
\end{abstract}




\begin{abstract}
ABRÉGÉ
Des calculs de dose précis et des outils d'analyse sont essentiels à la planification de traitement de radiothérapie. Il est accepté que les résultats cliniques peuvent être améliorés si l'exactitude dans la dose donnée au patient est améliorée. Il est prouvé que les calculs de dose par techniques Monte Carlo augmentent l'exactitude de la planification cependant, la plupart des centres de radiothérapie n'emploient pas ces techhniquer. Le système de planification de traitement par Monte Carlo de McGill, MMCTP, fournit un environnement de logiciel flexible pour intégrer la planification des traitements conventionnels et plus modernes en utilisant des simlations Monte Carlo. MMCTP est basé sur une interface usager graphique, fonctionnant sur un poste de travail simple relié à un réseau pour les calculs prolongés. Diverses mesures et recalculs de dose ont été effectués pour valider le logiciel et pour s'assurer de sa fonctionnalité.
\end{abstract}


TABLE OF CONTENTS

ACKNOWLEDGEMENTS ....................... ii

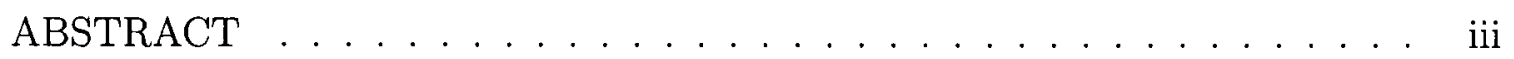

ABRÉGÉ ..................... iv

LIST OF TABLES . . . . . . . . . . . . . . . . . . viii

LIST OF FIGURES . . . . . . . . . . . . . . . . . . ix

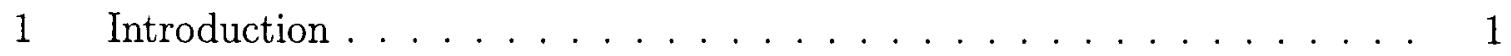

1.1 Medical Physics . . . . . . . . . . . . . . . . . . 1

1.2 Radiation Therapy . . . . . . . . . . . . . . . 2

1.2.1 External Beam Radiotherapy Equipment . . . . . . . . 3

1.3 Dose Accuracy for Radiation Therapy . . . . . . . . . . . . 3

1.4 Treatment Planning . . . . . . . . . . . . . . . . . 6

1.5 Rationale and Structure of Thesis $\ldots \ldots \ldots \ldots$

2 Basic Concepts of Radiation Physics . . . . . . . . . . . . . 9

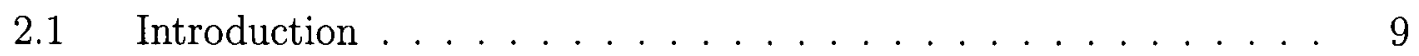

2.2 Basic Radiation Physics . . . . . . . . . . . . . . . . . . . 9

2.2 .1 Electron Interactions $\ldots \ldots \ldots$

2.2 .2 Photon Interactions . . . . . . . . . . . . . . . . 12

2.3 Radiation Dosimetry . . . . . . . . . . . . . . . . . 15

2.3 .1 Particle Fluence . . . . . . . . . . . . . . . . 15

2.3.2 Energy Fluence . . . . . . . . . . . . . . . . . 16

2.3.3 Attenuation of Photons . . . . . . . . . . . . . . 16

2.3 .4 KERMA . . . . . . . . . . . . . . . . . . 17

2.3 .5 Absorbed Dose . . . . . . . . . . . . . . . . . 18

2.3.6 Basic Dosimetric Functions . . . . . . . . . . . . . 19

2.4 Conventional Treatment Planning Systems . . . . . . . . . . 20

2.4 .1 Overview . . . . . . . . . . . . . . . 20 
2.4 .2 Dose Calculation Algorithms . . . . . . . . . . . . . . . 21

2.4.3 Plan Evaluation . . . . . . . . . . . . . . . . . 22

3 Monte Carlo Radiation Transport . . . . . . . . . . . . . . . . . . . 25

3.1 Transport Algorithm . . . . . . . . . . . . . . . 26

3.1 .1 Photon Transport . . . . . . . . . . . . . . . 27

3.1 .2 Electron Transport . . . . . . . . . . . . . . . 27

3.2 Statistics and Efficiency ................. 30

3.3 Electron Gamma shower Code (EGS) . . . . . . . . . . . . . 31

3.3.1 EGSnrc Code . . . . . . . . . . . . . . . . . . . . . . . . 32

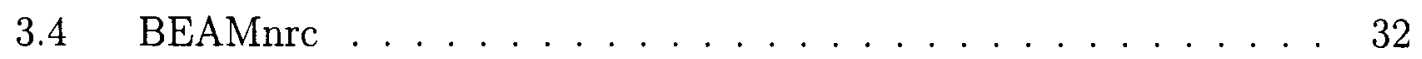

3.5 Voxel Monte Carlo (VMC, XVMC) . . . . . . . . . . . 35

4 Monte Carlo Treatment Planning . . . . . . . . . . . . . . . . . 37

4.1 Clinical MCTP systems . . . . . . . . . . . . . . . 37

4.2 Developing a MCTP system . . . . . . . . . . . . . . . 38

4.3 Commissioning a Monte Carlo Treatment Planning system . . . . 39

4.4 Normalization of Dose . . . . . . . . . . . . . . . . . . . . . 41

4.5 McGill Monte Carlo Treatment Planning . . . . . . . . . . . . . 42

5 MMCTP GUI for Monte Carlo Treatment Planning . . . . . . . . . . . . 45

5.1 Introduction . . . . . . . . . . . . . . . . 45

$5.2 \quad$ Philosophy and Design Decisions . . . . . . . . . . . . 47

5.2 .1 Software selection ................ . . . 48

5.3 MMCTP Programming . . . . . . . . . . . . . . . . 48

5.3 .1 McGill RT Format . . . . . . . . . . . . . . . . . . 49

5.3 .2 Monte Carlo Treatment Planning . . . . . . . . . . 58

5.4 MMCTP Modules . . . . . . . . . . . . . . . . . . 60

5.4 .1 Import/Conversion . . . . . . . . . . . . . . . . 60

5.4 .2 Treatment Planning . . . . . . . . . . . . . . 60

5.4 .3 Monte Carlo . . . . . . . . . . . . . . . . 62

5.4 .4 Contour Editor ................ . . 63

6 MMCTP Results and Validation . . . . . . . . . . . . . . 64

$6.1 \quad$ MMCTP GUI . . . . . . . . . . . . . . . . . 64

6.1 .1 Configurations folder . . . . . . . . . . . . . . 64

6.1 .2 Import Window . . . . . . . . . . . . . . . . . 68 
6.1.3 External Beam Window . . . . . . . . . . . . . . 70

6.1 .4 Contouring Window . . . . . . . . . . . . . . . . 77

6.1 .5 MMCTP Overview . . . . . . . . . . . . . 77

6.2 MMCTP Testing . . . . . . . . . . . . . . . 80

6.2 .1 DVH Verification ................ . . . . 80

6.2.2 Measurement Comparisons . . . . . . . . . . . . 85

6.2.3 Patient Recalculations . . . . . . . . . . . . . . . 92

7 Conclusion . . . . . . . . . . . . . . . . 98

7.1 Summary of work performed . . . . . . . . . . . . . . 99

7.1 .1 MMCTP GUI . . . . . . . . . . . . . . . . . . 99

7.1 .2 Validation ................... . . . . . . . . . . . . . . . . . . . . .

$7.2 \quad$ Future work . . . . . . . . . . . . . . . 101

References ......................... 103 


\section{LIST OF TABLES}

1-1 Estimated accuracy that is achievable and recommended in the delivery of an absorbed dose to the patient [19]. . . . . . . . . . . 5

4-1 Clinically available Monte Carlo Treatment Planning software. . . . . 38

6-1 DVH volume calculations; CADPlan calculated volumes are not as accurate as MMCTP volumes. As the volume size decreases to small volumes, the error increases. . . . . . . . . . . . . . . . . . . 84

6-2 Dose to water in $S A D$ setup at depth of $8.44 \mathrm{~cm}$ from measurements in Solid Water ${ }^{\mathrm{TM}}$ phantom and simulations . . . . . . . . . . 92

6-3 Dose to water in $S A D$ setup at depth of $8.44 \mathrm{~cm}$ from measurements in water tank and simulations . . . . . . . . . . . . . 93 
$\underline{\text { Figure }}$

\section{LIST OF FIGURES}

1-1 3D sketch of an external beam radiotherapy unit. The gantry, collimator and couch (table) are shown with their axis of rotation. The $X, Y, Z$ axis defines the patient coordinate system. . . . . 4

1-2 2D sketch of the collimater showing the $X_{1}, X_{2}, Y_{1}, Y_{2}$ jaw locations relative to the gantry and couch (table). . . . . . . . . 5

2-1 Coulomb interactions of an electron and atom. . . . . . . . . 10

2-2 Electron stopping power in liquid water as a function of energy.

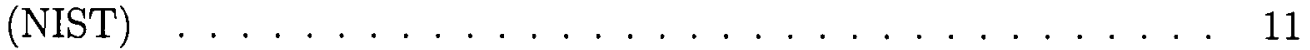

2-3 Cumulative DVH for target (-) and critical structure (- -): (a) ideal case (b) realistic case. . . . . . . . . . . . . . . . . . . . . 24

3-1 Monte Carlo photon transport algorithm [5] . . . . . . . . . 28

3-2 Electron transport path length correction; $\theta$ denotes the multiple scattering angle at the end of the step, $\psi$ the lateral deflection angle which is correlated to $\theta, \bar{t}$ is the straight line distance between the initial and final position of the electron, and $t$ is the curved path

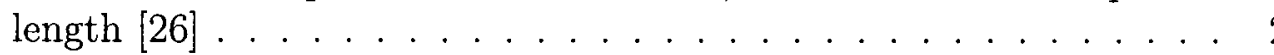

3-3 The structure of the EGSnrc code system when used with a usercode. [27] . . . . . . . . . . . . . . . . . 33

3-4 (a) Schematic drawing of linac components modeled in BEAMnrc Monte Carlo simulations [21], (b) BEAMnrc simulation of modeled CL21EX 18 MV photons (blue) through linac head.

4-1 Schematic diagram summarizing the generic components of a Monte Carlo treatment planning system [25]. 
4-2 Schematic diagram summarizing the validation steps in source or beam model and phantom and patient dose calculations in MCTP [25]. . 40

4-3 The relative increase in backscatter with decreasing field size for rectangular fields, with respect to a $40 \times 40 \mathrm{~cm}^{2}$ field for $10 \mathrm{MV}$ photon beams. Results are given for measurements (filled) and Monte Carlo simulations (open) for lower $X$ jaws (squares) fixed at a $40 \mathrm{~cm}$ field and upper $Y$ jaws (diamonds) fixed at $40 \mathrm{~cm} \mathrm{[22]} \mathrm{.} \mathrm{.} 43$

4-4 Block diagram of the steps involved for McGill Monte Carlo treatment planning system [7]. . . . . . . . . . . . . . . . . . . 44

5-1 McGill RT file structure, folders and files . . . . . . . . . . . . . 50

5-2 McGill RT image properties file: properties and their variable type. . 51

5-3 McGill RT patient coordinate system: The $(X, Y)$ origin is located at image centre with positive $X, Y$ directions as indicated. The $Z$ axis does not have a specific positive direction. . . . . . . . . . 52

5-4 McGill RT structure file: Example structure file, header information followed by struture points. . . . . . . . . . . . . 53

$5-5$ McGill RT beam geometry file . . . . . . . . . . . . . 55

$5-6$ McGill RT axial dose orientation . . . . . . . . . . . . . 56

5-7 McGill RT dose header block . . . . . . . . . . . . . . . . 57

5-8 McGill RT Monte Carlo settings file . . . . . . . . . . . . . . . . 58

5-9 MMCTP data flow, input files to cluster and $d 3 d$ file to work station . 59

6-1 mmctp.configuration: There are 3 sections to this file, electron, photon, and wedges. The electron and photon section has one line for each linac configuration. The linac name is first, followed by the energy, applicator for electrons, and MLC. The wedges are defined with the wedge angle and the wedge orientation options.

6-2 XVMC_dose.txt: The linac name is first, followed by the energy and the VMC dose per particle at the calibration point. The last line in the file is the correction for dose to water from dose to tissue. . . 
6-3 BEAM input file, jaw component module. $X, Y$ jaw positions . . . . . 67

6-4 BEAM input file, wedge component module for a 15 degree wedge . . 68

$6-5$ input.vmc file . . . . . . . . . . . . . . . . . 69

6-6 Import Window, import list-box on left and McGill RT list-box on right. . . . . . . . . . . . . . . . 70

6-7 External Beam Window . . . . . . . . . . . . . . . 71

$6-8$ External beam tab ..................... . . 72

$6-9$ Image tab f . . . . . . . . . . . . . . . . . 73

$6-10$ BEAM tab . . . . . . . . . . . . . . . . 74

$6-11$ VMC tab ............................. 75

$6-12$ Dose tab .......................... 76

$6-13$ DVH window . . . . . . . . . . . . . . . 76

6-14 Contour window . . . . . . . . . . . . . . . . 78

6-15 MMCTP user flow chart . . . . . . . . . . . . . . . . . . . 79

6-16 Painted dose distribution with various dose values. This distribution has the same dimensions as the CT image dimensions, thus the calculated DVH matches the manual DVH . . . . . . . . 82

6-17 DVH comparison between dose distributions with different dimensions. (a) Dose distribution with same dimensions as CT image. (b) Dose distribution with different dimensions than CT image. The DVH in (a) matches the manual calculation while the DVH in (b) does not match because of the interpolation which averages the dose values .

6-18 DVHs calculated on CADPlan and MMCTP (a) CTV (b) Lung (c) External $(\mathrm{d})$ Heart . . . . . . . . . . . . . . . . . 86 
6-19 Simulated and measured dose distributions for $6 \mathrm{MV}$ photon beam, $10 \times 10 \mathrm{~cm}^{2}$ field size, $15^{\circ}$ wedge. (a) measured film distribution. (b) simulated distribution. (c) gamma map comparison between film and simulation, $\gamma$ values above 1 represent a disagreement between the film and simulation within the tolerance limits, $\gamma$ values above 1 are coloured red.

6-20 Simulated and measured dose distributions for $6 \mathrm{MV}$ photon beam, with jaws at $X_{1}=10.2 \mathrm{~cm}, X_{2}=4.3 \mathrm{~cm}, Y$ field $20 \mathrm{~cm}, 15^{\circ}$ wedge and MLC pattern. (a) measured film distribution (b) simulated distribution (c) gamma map comparison between film and simulation, $\gamma$ values above 1 represent a disagreement between the film and simulation within the tolerance limits, $\gamma$ values above 1 are coloured red. . . . . . . . . . . . . . . 90 90

6-21 Comparison between isodose distribution. (a) Isodose distribution from CADPlan. (b) Isodose distribution from Monte Carlo. . . . . 94

6-22 DVH comparison for patient recalculations between CADPlan and MMCTP (VMC). (a) Patient 1 VMC CTV DVH is slightly higher than CADPlan but within 1\%. (b) Patient 2 VMC CTV DVH is in excellent agreement with CADPlan. . . . . . . . . . . 96

6-23 Profile comparison for CADPlan and VMC. VMC has a higher penumbra, due to CADPlan's inability to accurately model MLC fields. 


\section{CHAPTER 1 \\ Introduction}

\subsection{Medical Physics}

Medical physics is an applied branch of physics dealing with the application of physics in medicine. Since the discovery of x-ray in 1895 by Wilhelm Roentgen radiation has been integrated into medicine for various applications. Roentgen, who won the first Nobel prize in physics in 1901, was acclaimed as discovering a medical miracle. Today there are 4 main branches of medical physics,

- Medical Imaging : diagnostic application of x-rays, gamma rays, ultrasonic radiation, radio frequency radiation and magnetic fields. Evaluation of medical health, image quality and measurements from the associated equipment use.

- Radiation Therapy : therapeutic application of x-rays, gamma rays, electrons and charged particle beams, neutrons and radiation from sealed radionuclide sources. Evaluation of medical health, image quality and measurements from the associated equipment use.

- Nuclear Medicine : therapeutic and diagnostic application of radionuclides, not including those used in sealed sources for therapeutic purposes. Evaluation of medical health, image quality and measurements from the associated equipment use. 
- Health Physics : the safe use of x rays, gamma rays, electrons and other charged particle beams of neutrons or radionuclides and of radiation from sealed radionuclide sources for all applications except applications of radiation to patients for diagnostic or therapeutic purposes.

\subsection{Radiation Therapy}

Radiotherapy is a cancer treatment modality used to relieve symptoms and control the progression of cancer cells. Radiotherapy attempts to destroy cancer cells with ionizing radiation, while sparing healthy tissue. Ionizing radiation deposits energy along its path length to the medium, which can cause double strand DNA breaks. If a cell cannot repair a DNA break, the cell is considered dead and will not replicate. The probability of cell death is correlated to the amount of energy absorbed per unit mass of tissue. The tumor lethal dose, required to achieve $95 \%$ tumor control probability, depends on the cell radiosensitivity and radiation type. The tumor volume, location and cell type dictate the type of treatment chosen. The first case of radiation therapy was reported in 1899 , just a few years after the discovery of x-rays. Today about $50 \%$ of cancer patients are treated with radiation. Radiotherapy is divided into two types depending on the radiation source location. External radiotherapy involves a radiation source, which is external to the patient. The second type, brachytherapy, involves internal or external radiation where radioactive sources are placed in proximity of the tumor or lesion.

The primary challenge in radiotherapy is the trade off between destroying cancer cells and damaging healthy tissue. Radiation fields conform to the dimensions of the tumor or lesion to minimize the dose to healthy tissue, but the irradiation of some 
tissue is unavoidable. A second complication arises from the dose response curves. In an ideal world, the tumor is more sensitive or has less ability to repair damage than the surrounding tissue. In our world, this is not the case. Normal tissue complications occur at lower doses than tumor control. Thus, in radiotherapy, the dose to the tumor is limited by the dose given to surrounding tissues. Some of the side effects of radiotherapy include fatigue, skin reactions or hair loss. These effects are primarily due to the loss of normal tissue. Recent advances in radiotherapy aim at minimizing the dose to normal tissue while maintaining or escalating the tumor control. Some of these techniques include external 3D conformal radiation therapy (3D-CRT) or intensity modulated radiation therapy (IMRT) and multiple isocentrical beams.

\subsubsection{External Beam Radiotherapy Equipment}

External beam radiotherapy can be broken into two types, radioisotopes teletherapy machines and linear accelerators (linacs). At first glance, these two types appear similar in design. Treatment units are often designed with the source mounted on a rotating gantry, a rotating collimator and a rotating patient couch. These three components (gantry, collimator and couch) all rotate about the same point defined as the isocenter. A schematic sketch of a typical treatment unit is shown in Figure 1-1 and a view of the collimator is shown in Figure 1-2. The collimator consists of four jaws $\left(X_{1}, X_{2}, Y_{1}, Y_{2}\right)$, which form a rectangular field of dimension $X \times Y$.

\subsection{Dose Accuracy for Radiation Therapy}

In radiation therapy, accurate dose delivery is critical because of the steep relationship between dose and tumor control. A small change in the dose delivered 


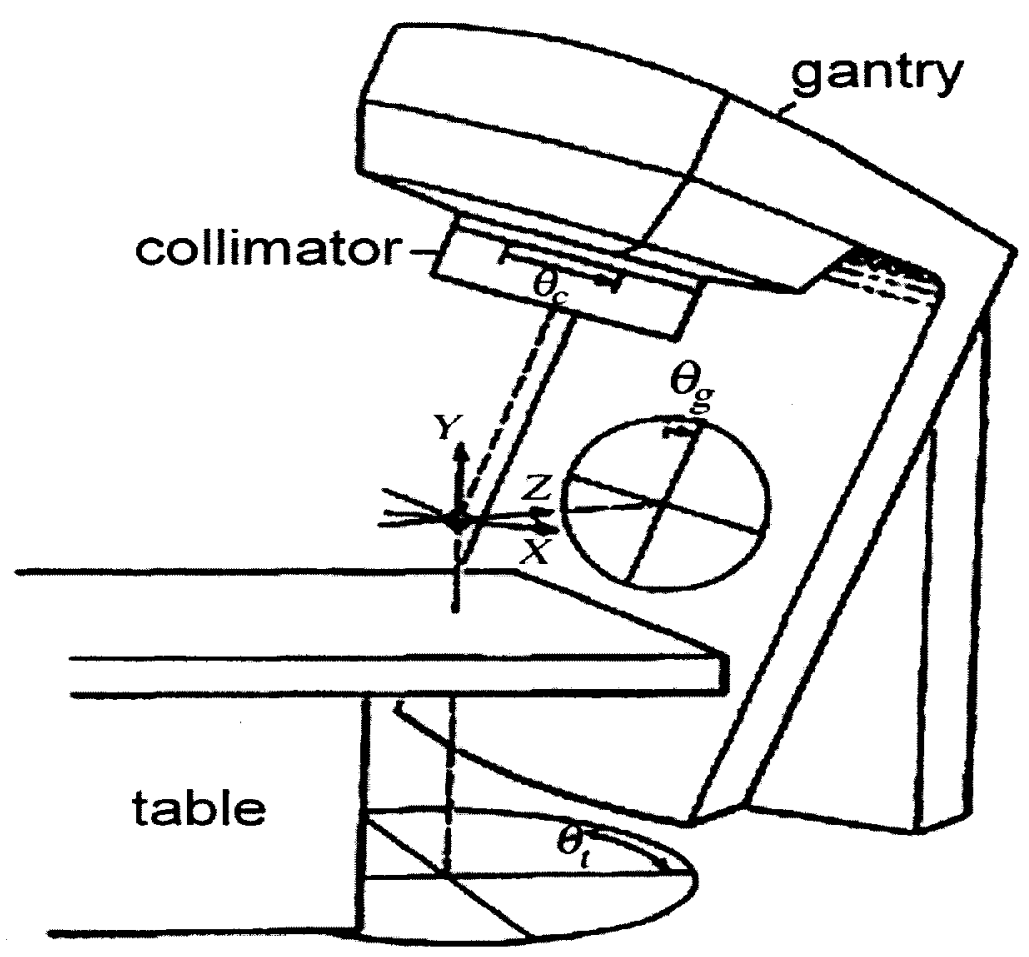

Figure 1-1: 3D sketch of an external beam radiotherapy unit. The gantry, collimator and couch (table) are shown with their axis of rotation. The $X, Y, Z$ axis defines the patient coordinate system.

can have a dramatic effect on patient outcome. Various studies have reported dose deliver accuracy and overall, it appears that the radiation dose can be determined in the patient to an accuracy ranging from between 4 and $8 \%$ [19] [4]. An overall dose uncertainty of $5 \%$ for the dose delivered to patients is recommended in ICRU report 24. An uncertainty of $5 \%$ is considered reasonable by ICRU report 24 for consistent clinical trails. Achieving an uncertainty of $5 \%$ is not a simple task and there are many procedures a department will follow to achieve this value. The four main types of uncertainties are summarized in Table 1-1. 


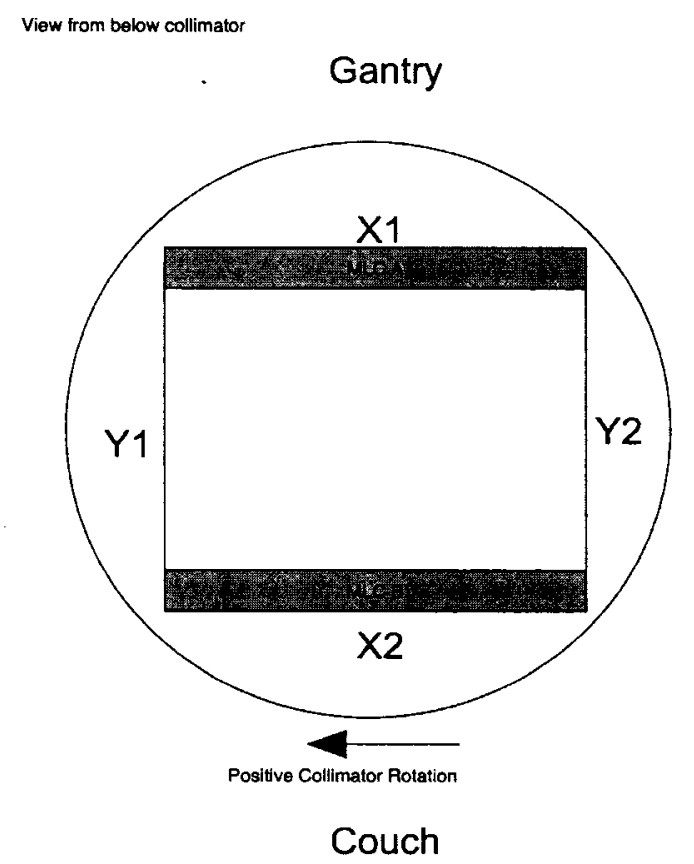

Figure 1-2: 2D sketch of the collimater showing the $X_{1}, X_{2}, Y_{1}, Y_{2}$ jaw locations relative to the gantry and couch (table).

Table 1-1: Estimated accuracy that is achievable and recommended in the delivery of an absorbed dose to the patient [19].

\begin{tabular}{lc}
\hline \hline Uncertainty & Recommended Accuracy (\%) \\
\hline Absorbed dose to a reference point in water phantom & 2.5 \\
Determination of relative dose (Measurement & \\
away from reference point) & 2.5 \\
Relative dose calculations & 2.5 \\
Patient irradiation & 2.5 \\
\hline Overall & $\mathbf{5 . 0}$ \\
\hline \hline
\end{tabular}

Over the last 10 years there have been improvements in the lower limit of these uncertainties. Improvements in calibration techniques and protocols have reduced the calibration uncertainty to within $1 \%$. Imaging improvements have decreased the patient data acquisition and patient setup. Patients are often imaged on the 
radiotherapy bed to correctly position patients before irradiation. The relative dose calculations are performed on advanced 3-dimensional treatment planning systems, which utilize patient data and the specifics of the treatments machines. Even the most advanced systems still have uncertainties between 3-4 \%. These large uncertainties arise because the treatment planning software does not accurately model the radiation output from the treatment machines or the density variations (inhomogeneities) within the patient. Inhomogeneity corrections can be applied to specific cases when necessary, but these corrections can sill lead to a high uncertainty. As a result, an overall uncertainty goal of $5 \%$ is difficult to achieve.

\subsection{Treatment Planning}

Treatment planning encompasses the efforts of the radiation oncology department to develop a patient specific delivery technique. Upon the diagnosis of cancer, the oncologist delineates the volumes and localization of targets on Computed Tomography (CT) images. These volumes include the GTV, CTV, and PTV. The gross target volume (GTV) is defined as the gross palpable or visible extent and location of malignant growth [2]. The clinical target volume (CTV) is defined as the tissue volume that contains a demonstrable GTV and or sub-clinical microscopic malignant disease, which has to be eliminated. This volume has to be treated adequately in order to achieve the aim of therapy cure or palliation [2]. While the planning target volume (PTV) is defined as the geometric concept that takes into consideration the net effect of all possible geometric variations in order to ensure that the prescribed dose is actually absorbed within the CTV [2]. The oncologist then prescribes a dose 
to the planning target volume and sets dose limits to the surrounding critical structures. The treatment planner uses the delineated CT images, the prescribed dose and the dose limits to develop beam configurations, which satisfy these conditions. The dose distribution is computed from the beam configurations and pre-calculated photon dose data sets. Often, the planner will edit the beam configurations to produce a higher conformity dose distribution. Under certain conditions, the treatment planner may decide to use inhomogeneity corrections to calculate the patient dose distribution.

An accurate treatment planning system must consider the exact patient data including the attenuation and scattering coefficients of each tissue inside the body. In addition, the treatment planning system must include the treatment machine's beam definition including attenuation and scattering coefficients of each internal component. Treatment planning systems do not consider this information with the exception of Monte Carlo treatment planning. Current treatment planning systems are either model or data based. Data based systems use measured data sets while model based systems use pre-calculated kernels to simulate the dose distribution. Monte Carlo is one model based method to simulate the dose distributions through explicit radiation transport algorithms which use random numbers to sample the interaction cross-section and probability distributions for particle interactions. By explicitly simulating the particles emerging from the accelerator head to the patient, the Monte Carlo simulation provides a more comprehensive and accurate dose calculation in the medium. Monte Carlo obviates the need for pre-calculated data sets and approximations. The dose calculation is now accurate and patient specific. 


\subsection{Rationale and Structure of Thesis}

The aim of this thesis is to design a computational treatment planning research graphical user interface (GUI) which incorporates new radiotherapy research techniques. Monte Carlo treatment planning is one technique that provides superior accuracy in dose calculation. The superior accuracy comes at the cost of increased calculation time. The main objective of this thesis is to design an interface to run Monte Carlo simulations in clinical use. Current Monte Carlo methods are often labor intensive and involve running various software programs. McGill Monte Carlo treatment planning (MMCTP) provides a simplistic treatment planning interface with automated Monte Carlo dose calculation. MMCTP also includes various treatment planning tools for visualization and dose analysis.

The following chapter introduces the basic concepts of radiation physics and radiation dosimetry. In Chapter 2, the approximations and limitations of convenetion radiation dosimetry are discussed. This leads into Chapter 3 where Monte Carlo techniques and Monte Carlo codes are introduced. The fourth chapter introduces the concept of Monte Carlo treatment planning. Chapter 5 introduces a graphical user interface for radiotherapy research with Monte Carlo treatment planning. Programing decisions and project goals are outlined in this chapter. Chapter 6 presents the finalized GUI and measurement validation results. This leads into the conclusion and future work section, which is discussed in Chapter 7. 


\section{CHAPTER 2 \\ Basic Concepts of Radiation Physics}

\subsection{Introduction}

This chapter introduces the fundamental interactions and effects from radiation physics. Radiotherapy relies on radiation physics and accurate treatment delivery for safe and accurate radiation treatment. The second part of this chapter will introduce radiation dosimetry and dosimetric functions for conventional treatment planning.

\subsection{Basic Radiation Physics}

Radiation is classified into two classes: non-ionizing and ionizing, depending on its ability to ionize atoms. The ionization potential, or the minimum energy required to ionize an atom ranges from a few $\mathrm{eV}$ for hydrogen to $24.6 \mathrm{eV}$ for helium. Ionizing radiation is used for radiotherapy treatments.

Ionizing radiation is divided into two types: directly and indirectly ionizing. Charged particles (electrons, protons, alpha particles and heavy ions) are directly ionizing radiation, while neutral particles (photons, and neutrons) are indirectly ionizing. The method of energy depositions is distinct between these types. Directly ionizing radiation deposits energy through Coulomb interactions with the medium. Whereas, indirectly ionizing radiation deposits energy through a two-step process. The first step releases a charged particle in the medium from a photon or neutron interaction. In the second step, the charged particle deposits energy through Coulomb interactions with the medium. 


\subsubsection{Electron Interactions}

As electrons travel through medium, they experience many Coulomb interactions with atomic nuclei and orbital electrons of the medium before they come to rest. Coulomb interactions arise because of the fundamental Coulomb force between two charged particles. These interactions are divided into three types depending on the impact parameter $b$ compared to the classical atomic radius $a$.

i Interactions with the external nuclear field $b<<a$ (Radiative or Bremsstrahlung)

ii Interactions with orbital electrons $b=a$ (Hard collisions)

iii Interactions with orbital electrons $b>>a$ (Soft collisions)

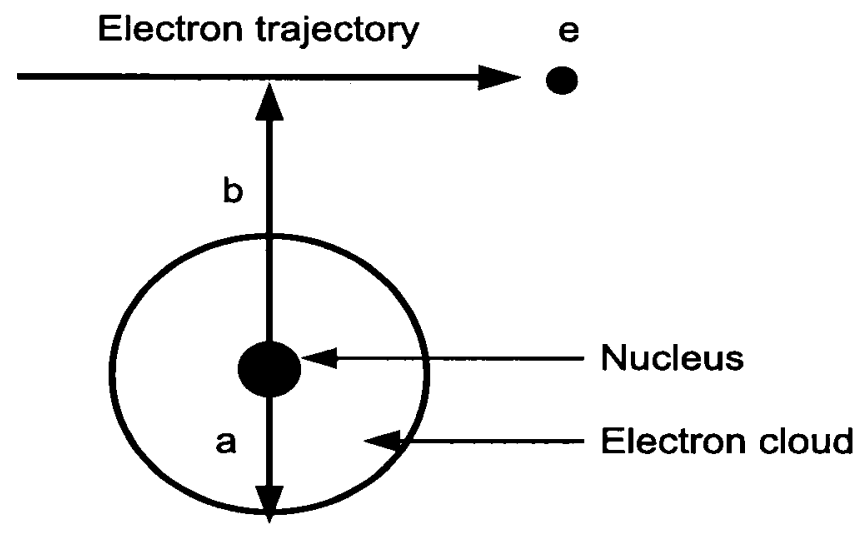

Figure 2-1: Coulomb interactions of an electron and atom.

At each interaction, the electron's path may change and depending on the interaction, it may lose kinetic energy. The energy loss is transferred to the medium (collisional loss), or to a photon (radiative loss). The rate of energy $(E)$ loss in a medium (collisional plus radiative) is called the linear stopping power $\left(\frac{d E}{d x}\right)$. The material density influences the stopping power, thus it is typically stated in $\mathrm{MeVcm}^{2} \mathrm{~g}^{-1}$, which is the linear stopping power $\mathrm{S}$ divided by the material density. There are two 
types of stopping powers: radiative and collisional. Radiative stopping power involves the interaction of a charged particle with atomic nuclei. This interaction generates a photon from the energy loss and the photon is referred to as a bremsstrahlung photon. Collisional stopping power involves the interaction of a charged particle with an orbital electron. These interactions transfer energy from the charged particle to orbital an electron. The total stopping power is the sum of radiative and collisional.

\section{WATER, LIQUID}

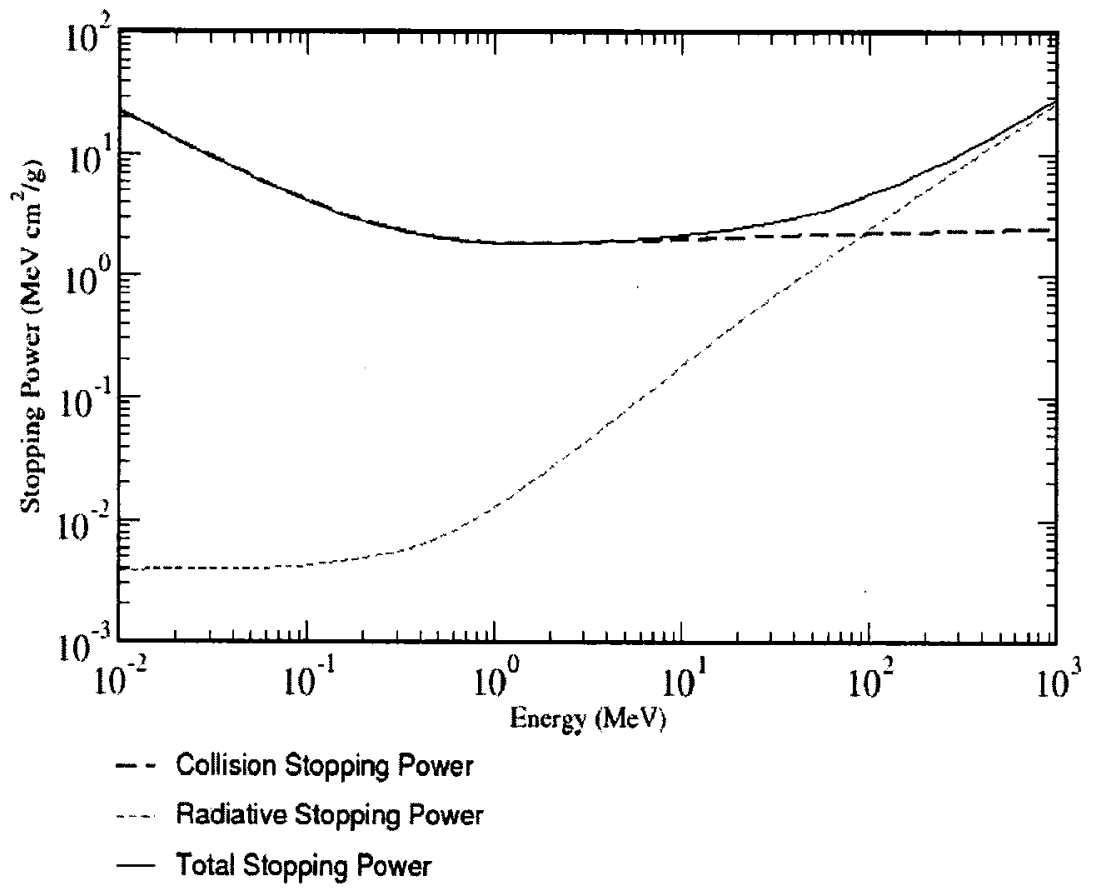

Figure 2-2: Electron stopping power in liquid water as a function of energy. (Reference NIST) 


\subsubsection{Photon Interactions}

Photons may experience various interactions while penetrating an absorbing medium. These interactions involve either the atomic nuclei or the orbital electrons of the medium. The photon interactions in the energy range of radiotherapy are predominantly photoelectric effect, Compton scattering, Rayleigh scattering and pair production. Radiotherapy is primarily concerned with calculating the energy transferred to the medium from these interactions.

\section{Photoelectric Effect}

The photoelectric effect is as an interaction between a photon and a tightly bound orbital electron of the absorbing medium. The interaction eliminates the photon and transfers its energy to the electron, thus, ejecting the electron from the atom with kinetic energy $E_{k}$. The kinetic energy of the ejected electron equals the incident photon energy minus the binding energy of the orbital electron. If the binding energy exceeds the photon energy, the electron remains bound to the atom but may rise to a higher orbit. The orbital vacancies resulting from these effects are filled by electrons in higher shells. This transition process emits either a characteristic photon or an Auger electron. With the atomic cross-section data and energy of the incident photon, one can calculate the mean energy transfer to these electrons. The general relationship for mean energy transfered to electrons $\left(\bar{E}_{t r}\right)$ is given by Equation 2.1 where $h v$ is the incident photon energy:

$$
\bar{E}_{t r}=h v-P_{k} \omega_{k} \bar{h} v_{k}
$$


Where $P_{k}$ is the fraction of all photoelectric effect interactions that occur in the K-shell. $\omega_{k}$ is the fluorescent yield for the K-shell and $\overline{h v_{k}}$ is the average energy transferred from the photon to the electron. The probability of photoelectric effect depends on the atomic number $Z$ and the energy of the incident photon. The photoelectric effect has a high probability of occurring for low energy photons and high $Z$ materials and if the incident photon energy is at or slightly above the binding energy of the electron.

\section{Compton Scattering}

Compton scattering or Compton effect involves a photon interaction with a loosely bound orbital electron. This interaction produces a scattered photon $\left(h v^{\prime}\right)$ with less energy than the incident photon $(h v)$ and an ejected electron referred to as a recoil electron. The loosely bound electron is considered free thus the recoil electron kinetic energy $\left(E_{k}\right)$ is equal to the energy difference between the incident photon and scattered photon. The recoil electron angle is the angle between the incident photon direction and the direction of the recoil Compton electron. There is also a scattering angle $(\theta)$ between the scattered photon and the incident photon direction. Three relationships : conservation of total energy, conservation of momentum in $x$ axis direction and $y$ axis direction, describes the Compton effect. Using these relationships one obtains the expressions for the scattered photon energy $h v^{\prime} \cdot 2.2$ and recoil electron kinetic energy $E_{k} 2.3$.

$$
h v^{\prime}=h v \frac{1}{1+\epsilon(1-\cos \theta)}
$$




$$
E_{k}=h v \frac{\epsilon(1-\cos \theta)}{1+\epsilon(1-\cos \theta)}
$$

Where $h v$ is the incident photon energy and $\epsilon$ is the normalized incident photon energy. The energies of the scattered electron and photon depend on their scattering angle thus angular probability of each. These angular probabilities are governed by the quantum mechanical theory of angular distributions described by Klein-Nishina. In general, the Compton scattering probability depends only on electron density and is the dominant photon interaction in water within the energy range $20 \mathrm{keV}$ to 10 $\mathrm{MeV}$.

\section{Rayleigh scattering}

A Rayleigh scattering event involves a photon interaction with a bound orbital electron: The event is elastic in the sense that the photon loses essentially none of its energy and is scattered through only a small angle. Rayleigh scattering transfers no energy to the charged particles but this process does contribute to the attenuation coefiicient.

\section{Pair Production}

The forth major photon interaction is pair production. Pair production can only occur if the incident photon energy exceeds $2 m_{e} c^{2}(1.022 \mathrm{MeV})$ and the photon enters the atomic nuclear field. In this interaction, the photon is converted into an electron-positron pair. The interaction transfers energy to both the electron and positron. Generally, the electron and positron do not receive the same kinetic energy but the average energy transferred $\bar{E}_{k}$ to each particle is given by Equation 2.4 where $h v$ is the incident photon energy. 


$$
\bar{E}_{k}^{p p}=\frac{h v-2 m_{e} c^{2}}{2}
$$

As the position travels and interacts in the medium, it slows down to very low energies before it combines with another electron to form a particle called a positronium. This particle exists for a short amount of time before it annihilates producing two photons each of energy $0.511 \mathrm{MeV}$. The probability of pair production increases with photon energy and increases with atomic number $Z$.

A sub set of pair production occurs when the photon enters the field of an orbital electron. This event, called triplet production, is considerably less probable and has a threshold energy of $4 m_{0} c^{2}$.

\subsection{Radiation Dosimetry}

Radiation dosimetry attempts to quantify absorbed dose through measurements or calculations. Dosimetry today is based on the quantity absorbed dose and accurate measurements of absorbed dose represent one of the major responsibilities of clinical medical physicists [14]. A number of concepts have been defined for quantifying absorbed dose at the macroscopic and microscopic level. Some of these terms will be introduced.

\subsubsection{Particle Fluence}

Particle fluence is the number of quanta per unit area. The fluence is defined as the number of particles $d N$ crossing an area $d A$ as:

$$
\Phi=\frac{d N}{d A}
$$

The unit of particle fluence is $\mathrm{m}^{-2}$. 


\subsubsection{Energy Fluence}

The energy fluence takes into account the radiation energy of the particles $E$, and particle fluence:

$$
\Psi=\frac{d E}{d A}
$$

The unit of energy fluence is $\mathrm{Jm}^{-2}$. For a monoenergetic photon beam with energy $E$, the energy fluence is given by:

$$
\Psi=\frac{d N}{d A} E
$$

\subsubsection{Attenuation of Photons}

Photons have a given probability of interaction depending on the photon energy and the traveling medium. These probabilities arise from calculated cross-section data. The cross-section reports the probability of an interaction per electron or atom for each interaction type. The total cross-section is the sum of each interaction cross-section. To calculate the probability of a photon interaction in the megavoltage energy range one uses the total cross-section $\mu$ :

$$
\mu=\tau+\sigma+\kappa
$$

Where $\tau, \sigma, \kappa$ are the cross-section symbols for photoelectric effect, Compton scattering and pair production. The total cross-section is used to calculate the change in particle fluence and energy fluence as photons travel through materials. The 
change in particle fluence of an incident photon beam after traveling a distance $d x$ is:

$$
d \Phi=\Phi \mu d x .
$$

Similarly, the change in energy fluence is:

$$
d \Psi=\Psi \mu d x
$$

The attenuation for a beam of photons after travel through a material of thickness $x$ becomes:

$$
\Psi(x)=\Psi_{0} e^{-\mu x},
$$

where $\Psi_{0}$ is the initial energy fluence.

\subsubsection{KERMA}

KERMA is an acronym for Kinetic Energy Released per unit MAss. In the case of indirectly ionizing radiation such as photons, KERMA quantifies the average amount of energy transferred from indirectly ionizing radiation to directly ionizing radiation. The SI KERMA unit is $\mathrm{Jkg}^{-1}$. Energy from photons is transferred in a two-step process. First, energy is transfer to electrons as described in Section 2.2.2. The electrons transfer their energy to the medium through atomic excitations and ionizations. Hence, KERMA is the calculation of mean energy transferred to charged particle per photon interaction per unit mass. The general equation for KERMA is given by: 


$$
K=\frac{d \bar{E}_{t r}}{d m},
$$

where $d \bar{E}_{t r}$ depends on the incident photon energy and atomic number of the medium. As mentioned earlier, the energy transferred to charged particles can be expended through collisional and radiative interactions. Therefore, the total KERMA is divided into two components, the collisional KERMA $K_{\text {coll }}$ and radiative KERMA $K_{\text {rad }}$. The average fraction of energy transferred to electrons that produce radiative energy is represented as the radiative fraction $\bar{g}$. This leaves the fraction lost through collisional interactions to be $(1-\bar{g})$. A useful relation between collisional KERMA and total KERMA $K$ may be written as:

$$
K_{\text {coll }}=K(1-\bar{g}) .
$$

\subsubsection{Absorbed Dose}

The absorbed dose $D$ is defined at a point inside a volume as the mean energy imparted by ionizing radiation to matter of mass $m$.

$$
D=\frac{d \bar{E}}{d m}
$$

The energy imparted is determined by taking the sum of energy entering the volume and subtracting the energy leaving the volume. The unit of absorbed dose is joule per kilogram, and its give a special name of Gray (Gy) which is equivalent to $1 \mathrm{Jkg}^{-1}$. 


\subsubsection{Basic Dosimetric Functions}

Dosimetric functions are used to approximate the dose within phantoms or patients. These functions relate the dose at any arbitrary point in a phantom as a percentage of the reference dose point. The reference point is chosen along the beam central axis at the point of maximum dose $\left(z_{\max }\right)$ in a field that is $10 \times 10 \mathrm{~cm}^{2}$ at 100 $\mathrm{cm}$ source to surface distance $(S S D)$. This reference point is also the monitor unit calibration point. A linac is always calibrated to deliver $1 \mathrm{cGy}$ to the reference point per monitor unit (MU). Where $1 \mathrm{MU}$ corresponds to a relative amount of charge collected by a monitor chamber within the linac. Clinics will often use lookup tables of relative dose fraction or other functions for quick dose assessment.

Percentage Depth Dose $(P D D)$

This dosimetric function relates the dose delivered along central axis of a patient or phantom relative to the dose at $z_{\max }$. The PDD is defined as:

$$
P D D(z, A, f, h v)=100 \frac{D_{Q}}{D_{p}},
$$

where $D_{Q}$ is the dose at point $Q$ depth $z$ on the central axis while $D_{p}$ is the dose at point $P$ at $z_{\max }$ on the central axis. The $P D D$ is a function of depth $z$, field size $A$, source to surface distance $(S S D) f$ and beam energy $h v$. $P D D$ tables for common energies, depths and fields sizes are published in supplement 25 of the British Journal of Radiology [1]. For a typical photon beam, the PDD curve exhibits a rapid increase until the depth of maximum dose $z_{\max }$. Beyond this depth, the $P D D$ curve decreases exponentially. 


\section{Tissue Maximum Ratio (TMR)}

The $T M R$ ratio was introduced for use in megavoltage isocentric setups. The $T M R$ is the ratio of dose at one depth $\left(D_{Q}\right)$ to the dose at the reference depth $\left(D_{Q_{\max }}\right)$. The reference depth is chosen to be the depth of maximum dose. This function is most commonly used for dosimetry applications. Most clinics have tabulated $T M R$ values where one can quickly determine the dose at various depths in isocentric setups.

$$
\operatorname{TMR}\left(z, A_{Q}, h v\right)=\frac{D_{Q}}{D_{Q_{\max }}}
$$

\subsection{Conventional Treatment Planning Systems}

\subsubsection{Overview}

Treatment planning systems (TPS) are complex software packages that are used to design and optimize radiation treatments. These systems calculate a patient dose distribution based on the 3D patient data set and the prescribed treatment plan. The first step to generating a patient plan is acquiring 3D patient data. Patient data is usually in the form of CT scans or some combination of CT and magnetic resonance imaging (MRI) or positron emission tomography (PET) imaging. Once the patient data is within the planning system, an oncologist can delineate the volumes of interest. These volumes include the planning target volume (PTV) and the various organs at risk (OAR). After delineating the volumes, radiation beams are placed at various angles around the PTV. The shape and placement of these beams is often optimized to produce a homogeneous dose distribution throughout the PTV while 
sparing the dose to the OAR. The patient dose distribution is calculated from basic dosimetry functions generated from water phantom measurements.

\subsubsection{Dose Calculation Algorithms}

The dose calculation algorithm defines the treatment planning system. Algorithms are classified into correction-based and model-based. Both approaches require a considerable amount of water phantom measurements as input data for commissioning the treatment planning system.

\section{Correction-based algorithms}

The correction-based algorithms, derived from standard dosimetric functions, attempt to correct for irregular shaped fields, oblique surfaces and heterogeneous material from the standard water phantom measurements acquired under regular fields with perpendicular beam incidence. These corrections are needed because conformal radiotherapy uses irregularly shaped fields directed towards oblique patient surfaces. A common correction for irregular shaped fields is the Clarkson method [11]. The Clarkson method is an integration of weighted regular shaped fields applied to any dosimetic function for its value in irregular shaped fields.

The corrections for heterogeneous materials due to patient anatomy are somewhat more complicated. Inhomogeneities produce changes in the dose distribution functions because of density variations. Density changes affect the attenuation of primary photons and the secondary electron fluence. There are a few techniques to correct the dose for these effects. The most commonly used inhomogeneity corrections are Batho power law [23], modified Batho power law and equivalent tissue air ratio (EqTAR). These corrections work well for simple phantoms with low energy 
beams but they do not consider the variation of secondary electron fluence within the inhomogeneous media. As a result, accurate patient dosimetry in high energy beams is compromised while using these methods.

\section{Model-based algorithms}

Model-based algorithms use radiation transport models to calculate absorbed dose. These models are tweaked by standard water phantom measurements to accurately simulate the dose distribution. One widely used algorithm is the pencil beam convolution model [3]. This method divides the beam into many narrow beams, "pencils", and the dose deposition at a point is the superposition of all beams. The dose is calculated using scattering kernels for radiation transport to simulate scattered photons and secondary electrons. These kernels, which describe the energy deposition around a primary photon interaction site, are considered spatially invariant. The accuracy of the dose distribution relies on the accuracy of these kernels. The kernels are often calculated from Monte Carlo simulations in homogeneous water phantoms. In the case of heterogeneities, a correction is applied to the scattering kernel, which scales the kernel by the average density along its path. Pencil beam algorithms increase the accuracy over correction-based algorithms but they still rely on

an empirical correction method to account for heterogeneity corrections. Ultimately, this limits their accuracy [18].

\subsubsection{Plan Evaluation}

Once the TPS has calculated a dose distribution, it is necessary to assess the merit of the plan. There are various methods for plan evaluation, some of which include: 
- Dose volume histogram (DVH)

- Dose distribution statistics

- Isodose curves

- Colour wash dose distributions

DVHs are useful quantitative evaluation tools which summarize the 3-D dose distributions pertaining to the targets and volumes of interest. The DVH represents a frequency distribution of dose values within a defined volume. There are two ways of representing a DVH. Differential DVHs represent the frequency plot of the number of voxels within the structure of interest that receive dose in a given range. Cumulative DVHs plot the structure volume that receives at least a given dose as a function of dose. DVH are calculated for the target volumes and OAR and are often used to compare competing treatments plans. They also reveal the existence of under and over dose spots within a structure volume. The main drawback of the DVHs is the loss of spatial information that results from the condensation of data when the DVHs are calculated [?]. The ideal plan would produce a DVH with 100 $\%$ of the target volume receiving $100 \%$ of the dose and the OAR receiving $0 \%$ of the dose. Realistic and ideal DVH are shown in Figure 2-3.

Dose statistics provide quantitative information on the PTV or OAR and on the dose received by that volume. From the dose distribution, various statistics are calculated of each volume. These include:

- Minimum dose to volume

- Maximum dose to volume

- Mean dose to volume 


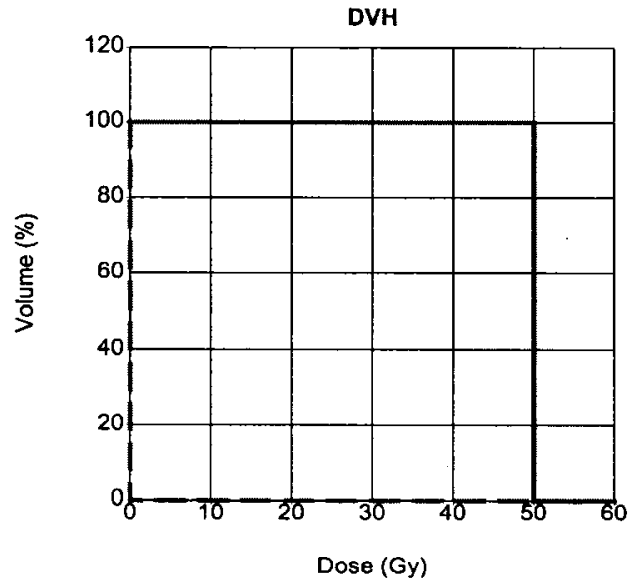

(a)

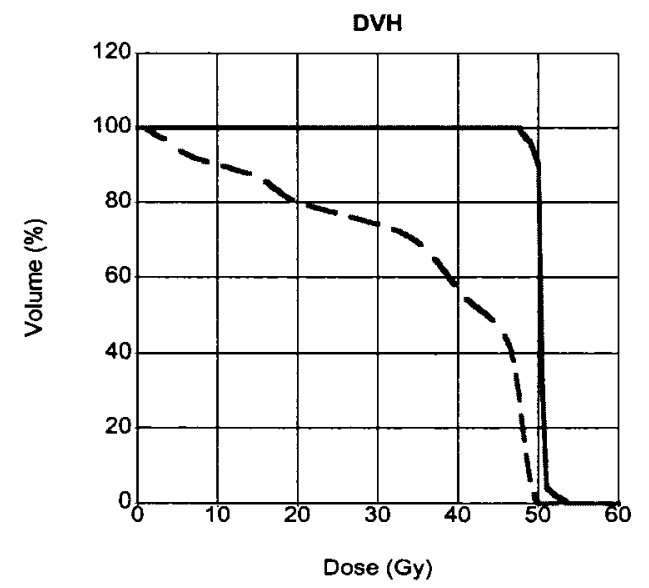

(b)

Figure 2-3: Cumulative DVH for target (-) and critical structure (- -): (a) ideal case (b) realistic case.

- Dose received by at least $x \%$ of the volume

The isodose curves and colour wash dose distributions graphically display the spatial distribution of dose in relation to the anatomy. Most often, they are superimposed over 2D axial, sagittal or coronal images. 


\section{CHAPTER 3 \\ Monte Carlo Radiation Transport}

Monte Carlo radiation transport is a technique that uses pseudo random numbers to simulate naturally random processes based on statistical probabilities. The idea was developed in the 1940's and its use has spread to many fields. One can find Monte Carlo techniques in the simulation of high-energy physics to the simulation of a Bingo game. The technique uses a pseudo random number generator to sample the probability distribution function of a physical system to arrive at a solution. For statistically significant solutions, a large number of histories are needed. Although, as the number of histories increase, the calculation time also increases. Thus, Monte Carlo calculations are computationally demanding. With the development of powerful computers, Monte Carlo techniques are now entering the field of radiotherapy.

In radiotherapy, Monte Carlo techniques simulate radiation transport from linear accelerators or radioactive materials. Radiation transport refers to the transportation of each original particle and its entire offspring to determine the dose deposited in media. As a particle travel through a medium, it creates a shower of secondary particles through interactions. These interactions follow specific probability distributions based on initial particle type and energy. For every particle, the Monte Carlo algorithm determines the location and type of interaction. These interactions lead to the deposition of energy along the particle path. As the particles interact, the information pertaining to the interaction type and location is saved as the particle 
history. The standard deviation, absorbed dose or fluence is determined after the simulation of many particles. Aside from the calculation of dose, Monte Carlo also offers the ability to calculate fluence, detector related quantities, electron contamination and fraction of primary or scattered dose. It is often useful to determine where the contributions of scattered dose originates. The main components of a Monte Carlo algorithm include cross-section data for the interaction probabilities, the transport algorithm, geometry specifications, and the data analysis tools. The transport algorithm determines the characteristics and efficiency of any Monte Carlo algorithm. Most often, cross-section data is included within the transport algorithm.

\subsection{Transport Algorithm}

Transport algorithms are needed to describe the coupled transport of photons and electrons or positrons through media. The parameters that describe the particles, such as charge, energy, position and direction are stored in stack memory. The algorithm begins with an initial number of primary particles. Each primary particle will generate a shower of secondary particles. These secondary particles are placed in the stack for additional transport. The transport terminates once the particle's energy falls below the cutoff energy or the particle exits the volume. At this point, the algorithm transports the next particle in the stack until there are no more remaining particles within the stack. The particle history is a list of all interaction events beginning from the initial primary particle down to the last remaining particle in the stack. Once the stack is empty, the algorithm begins to transport the next primary particle. This process continues until all particles are transported. 


\subsubsection{Photon Transport}

Unlike electrons and positrons, photons are uncharged particles and can travel large distances through a medium without interacting. The cross-section data for different interactions provide the statistical probability for photon interactions. These interactions involve one of the following effects:

- Photoelectric effect

- Compton effect

- Rayleigh scattering

- Pair and Triplet production

A typical photon transport algorithm is shown in Figure 3-1. The transport first determines a step length based on the mean free path for a photon and a random number between 0 and 1 . The photon is then transported to this new location and possibly discarded if the location lies outside the geometric volume. The next step is to determine the type of interaction at this location. A second set of random numbers samples the cross-sectional probability distribution to select an interaction type. At this point, the photons energy and direction are recalculated based on the interaction and any new particles created are added to the stack for further transport.

\subsubsection{Electron Transport}

Electron transport is more complex than photon transport because in the radiotherapy energy range, electrons can undergo upwards of $10^{6}$ interactions before they are absorbed. These interactions are divided into three categories:

i Coulomb force interaction with external nuclear field (bremsstrahlung)

ii Coulomb force interaction with orbital electrons (hard collisions) 


\section{Photon Transport}

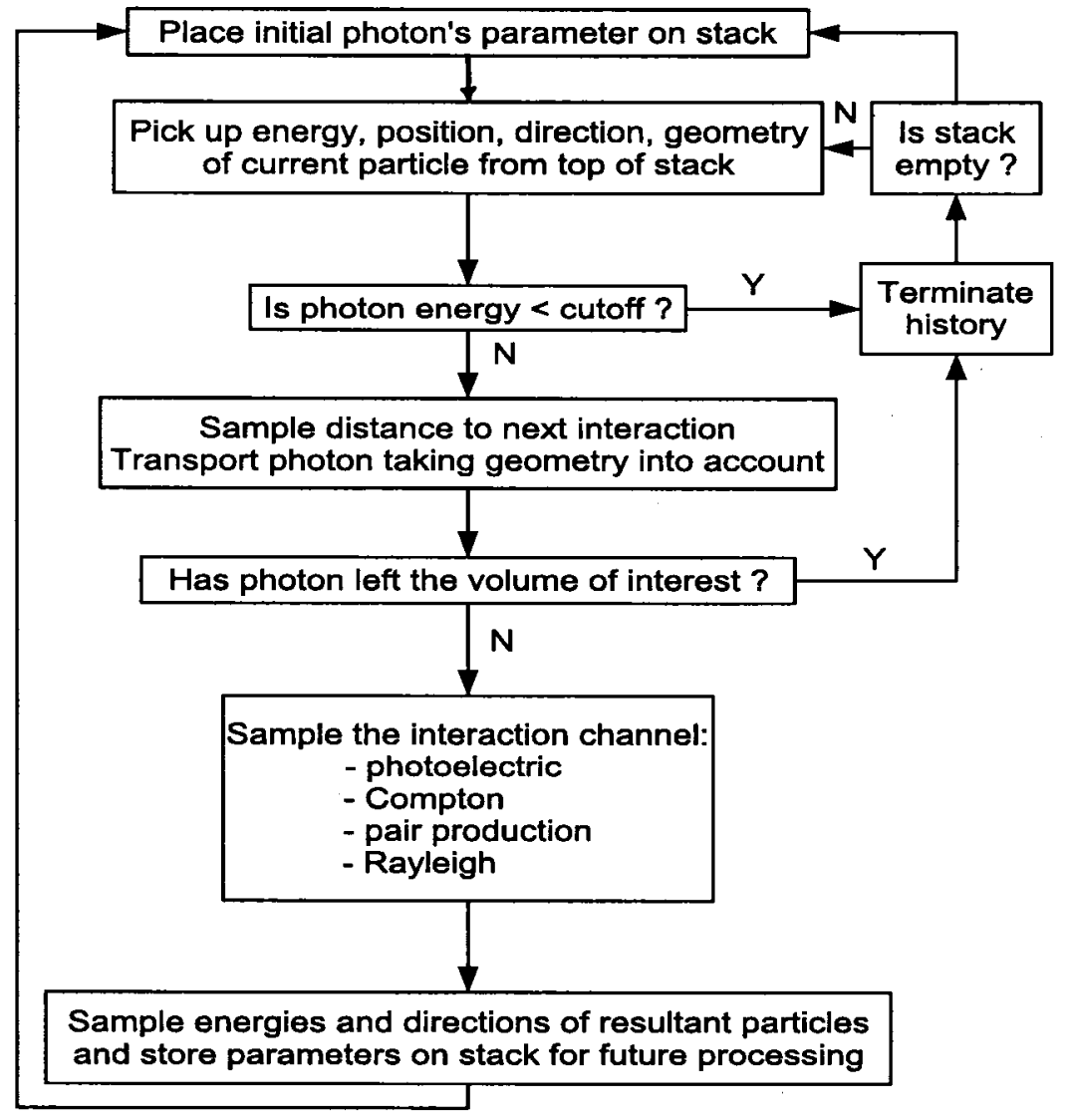

Figure 3-1: Monte Carlo photon transport algorithm [5]

iii Coulomb force interaction with orbital electrons (soft collisions)

A full simulation of these interactions, greatly increases the computational time. This is undesirable and impractical for medical applications. A new approach to perform full electron transport was proposed by Berger [8] in 1962 called the condensed history technique. This technique groups many interactions within one-step. The energy loss and angular directions are evaluated step by step. The electron transport 
from one step to another accounts for the combination of numerous interactions. At the end of each step, the deflection angle is determined from multiple scattering theory by sampling an angle from the multiple scattering distribution. For each step, the energy loss is approximated from the continuous slowing down approximation. The condensed history technique, as shown in Figure 3-2, can be summarizes by a step length, a lateral displacement and a scattering angle.

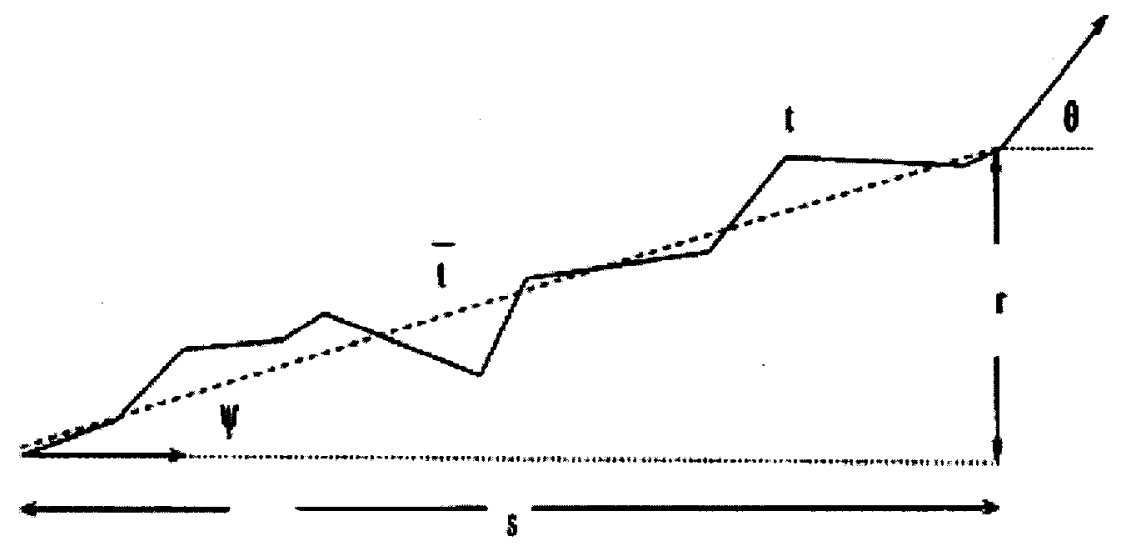

Figure 3-2: Electron transport path length correction; $\theta$ denotes the multiple scattering angle at the end of the step, $\psi$ the lateral deflection angle which is correlated to $\theta, \bar{t}$ is the straight line distance between the initial and final position of the electron, and $t$ is the curved path length [26]

Condensed history is accurate and computationally feasible. Similar to photon transport, the electrons are processed one at time. Secondary particles, knock-on electrons and bremsstrahlung photons, may be produced at each step according to the cross-sectional data. The step size changes depending on the proximity to material boundaries. As an electron crosses a boundary, the step size is kept small to accuratly simulate the process. A dynamic step size reduces the computational 
time while maintaining the accuracy. When the electron energy falls below the cut-off energy or exits the geometric volume, the transport is terminated.

There are two classes of condensed history algorithms for electron transport. Within class II, the creation of secondary particles (knock-on electrons and bremsstrahlung photons) affects the energy loss and angular direction of the primary electron. Class I ignores these effects and assumes the energy loss depends only on the unrestricted stopping power for each step length. In general, Class II algorithms are more accurate but different doses between these classes are rarely noticed [7].

\subsection{Statistics and Efficiency}

There are two types of uncertainty; type A and B. Type A uncertainties are of a statistical nature while type B are systematic in nature. For the evaluation of statistical uncertainties, one can use Gaussian or Poisson statistics to estimate the variance. Programming errors, cross-sectional data and general modeling inaccuracies belong to type B uncertainties. These uncertainties are not usually considered in Monte Carlo calculations.

The variance of type A uncertainties for Monte Carlo simulations can be written as:

$$
s_{<x>}^{2}=\frac{1}{N-1}\left[\frac{1}{N} \sum_{i=1}^{N} x_{i}^{2}-\left(\sum_{i=1}^{N} \frac{x_{i}}{N}\right)^{2}\right] .
$$

Where $x_{i}$ is the quantity scored in statistically independent event $i$ and $N$ is the number of independent events (histories) [9]. In the limit of a large number of histories $(N)$, the quantity $N s^{2}$ can be considered constant and the uncertainty can 
be approximated to $N^{-0.5}$. This leads to the following Monte Carlo definition of efficiency $\epsilon$.

$$
\epsilon=\frac{1}{T s^{2}}
$$

Where $T$ is the computing time required to obtain the variance $s^{2}$. The efficiency equation states that the efficiency of a particular simulation is constant. Therefore, a decrease in the statistical uncertainty of 10 requires an increase in the computation time of 100 . Variance reduction techniques reduce the statistical uncertainty while maintaining the same computational time. These techniques attempt to increase the relative occurrence of certain event, which minimize the number of required histories. There are a number of variance reduction techniques available for each Monte Carlo package. Forced interactions, particle splitting, cross-section enhancement, bremsstrahlung splitting and range rejection are some examples.

\subsection{Electron Gamma shower Code (EGS)}

The EGS [6] code is widely used in the field of physics because of its high accuracy radiation transport. The code, originally developed by Nelson and Jenkins in 1978 for applications in high-energy physics, was modified in 1985 to EGS4 [34] for improved accuracy in low energy radiation transport down to $1 \mathrm{keV}$. Radiotherapy treatments use low energy radiation $(0.5 \mathrm{keV}-20 \mathrm{MeV})$. Thus, EGS4 is ideal for radiotherapy. EGS4 is considered a class II algorithm where the production of secondary particles is treated on individual basis. Over the years, there have been improvements to the EGS4 PRESTA algorithm for increased accuracy with electron transport particularly when a particle travels between mediums. In addition, there 
are newly added features for example: bremsstrahlung angular sampling, fluorescent photons from the atomic shells, Auger and Coster-Kronig electrons and various variance reduction techniques.

EGSnrc [17] has evolved from EGS4 with many physics improvements. The EGSnrc code package simulates the cross-sectional data for various interaction probabilities. An algorithm called PEGS4 generates the cross-sectional data from materials ranging from $Z=1$ to $Z=100$ with energies ranging from a few keV up to $\mathrm{TeV}$. In addition to cross-sectional data, stopping power tables are required for particle transport. These tables are created from Berger and Seltzer's work based on the stopping powers formula from Sternheimer [31].

\subsubsection{EGSnrc Code}

As shown in Figure 3-3, the EGSnrc code is split into two sections; the user code and the EGS transport code. The user code consists of MAIN, HOWFAR, and HOWNEAR, which describes the input parameters and geometry for a simulation. The AUSGAB module defines the output quantities and variance reduction techniques. All input parameters are entered through MAIN module, which prompt the HATCH and SHOWER modules. HATCH reads the cross-sectional data produced from PEGS4 while SHOWER generates the complete particle history for each stack.

\subsection{BEAMnrc}

BEAMnrc [16] is a EGS user code developed through the OMEGA project [15] (Ottawa Madison Electron Gamma Algorithm) for the simulation of radiation beams from radiotherapy sources. The code performs the particle transport through the complex geometry of treatment units. Treatment units include linear accelerators, 


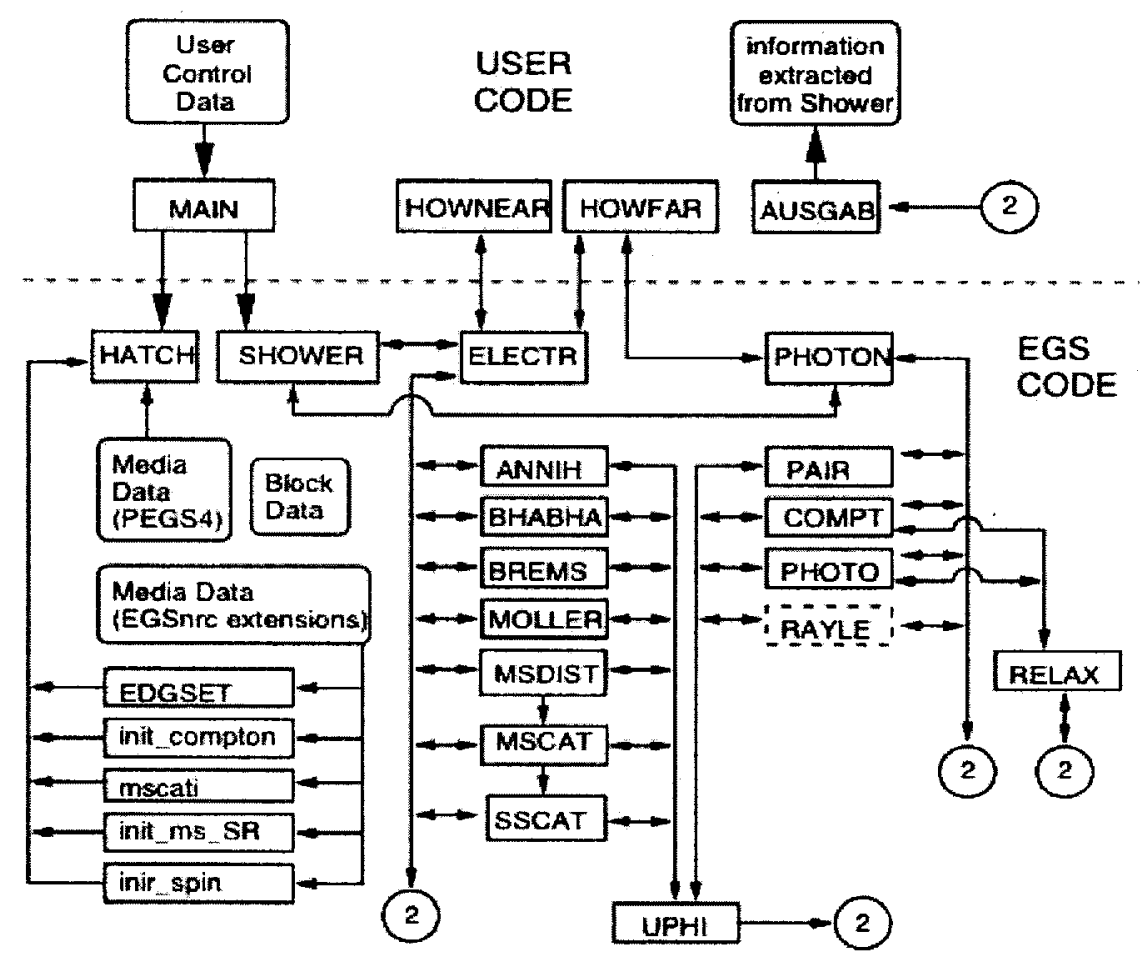

Figure 3-3: The structure of the EGSnrc code system when used with a usercode. $[27]$

orthovoltage units and Cobalt-60 units. BEAM uses the concept of component modules (CM) to model each component of a treatment unit. The CM modules are stacked perpendicular to the beam axis with a top and bottom surface. The dimensions and materials of each CM module is specified within the BEAM input file. A modeled treatment unit consists of a series of independent CMs sandwiched together. Some examples of CM are: air slabs, jaws, MLC and mirror. Aside from the CMs, the BEAM input file contains transport parameters regarding the energy cutoffs limits for electrons (ECUT) and photons (PCUT), minimum energy required for knock on electrons (AE) and bremsstrahlung photons (AP). In addition, the energy source 
(monoenergetic, spectrum or phase space file), and the variance reduction options, are included within the input file.

The BEAMnrc output is a phase space file containing information on the physical characteristics of all particles exiting the last CM plane. These characteristics include $x, y, z$ coordinates, direction, particle energy and particle weight. The phase space output can be calculated below any CM. In addition, the phase space can be used as a source for another simulation, or analyzed using user codes to calculate physical quantities. These quantities include: particle energy spectra, fluence, angular distributions and spatial distributions.

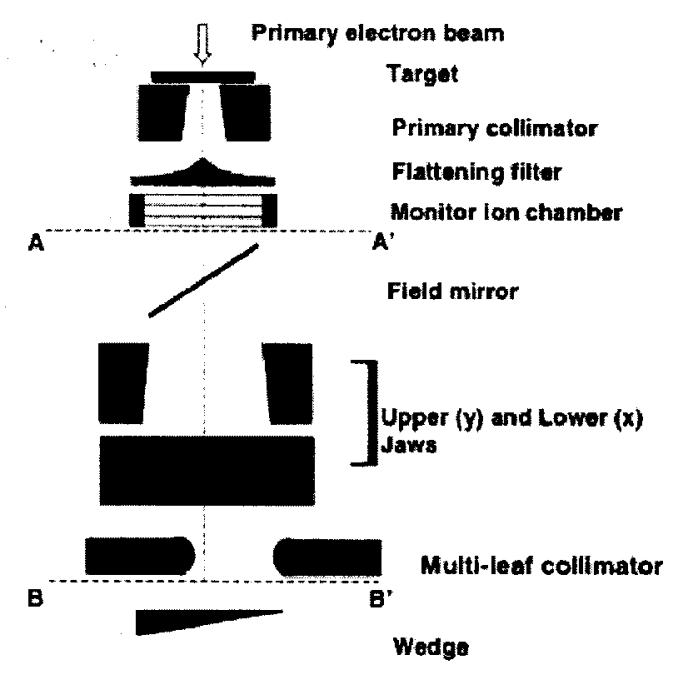

(a)

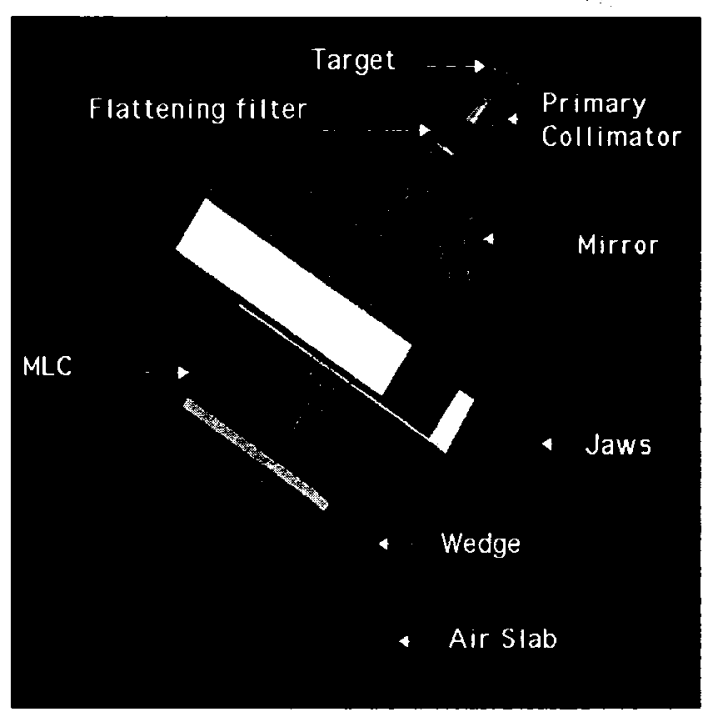

(b)

Figure 3-4: (a) Schematic drawing of linac components modeled in BEAMnrc Monte Carlo simulations [21], (b) BEAMnrc simulation of modeled CL21EX $18 \mathrm{MV}$ photons (blue) through linac head. 


\subsection{Voxel Monte Carlo (VMC, XVMC)}

Specialized codes have been designed to minimize unnecessary complications by simplifying Monte Carol simulations to specific limits. VMC [30] is a fast Monte Carlo algorithm written to simulate electron transport within the limits of radiotherapy. Unlike EGS4 which is designed for a wide range of energies and materials, VMC is applicable only within an energy range of 1-30 MeV and for low $Z$ materials with physical densities ranging from 0 to $3 \mathrm{gcm}^{-3}$. This is ideal for transporting particles through patients. These physical limitations allows for calculations 35 times faster than EGS4 and EGSnrc. A few approximations help speed up the calculations. These approximations include: multiple history technique and step size restriction for electron transport. Multiple history technique reuses the same electron history in different regions of the simulated volume. This reduces the number of histories required to achieve a certain statistical uncertainty thus, decreasing the calculation time. Step size restrictions decrease the number of electron steps per history by increasing the step size.

$\mathrm{XVMC}$ is a more recent version of VMC, which allows for coupled photon and electron transport and uses a ray tracing technique to calculate primary photon interactions in each voxel. The sources in XVMC can be monoenergetic electrons or photons, a spectrum, a beam model, or a phase space file. Similar to BEAM, the source is defined within the XVMC input file. The geometric volume or phantom is defined within the input file or within a density matrix file (DMX). The density matrix file is a matrix of voxels, where each voxel is assigned a specific density. The voxel dimensions represent the spatial resolution or the scoring volume. Decreasing 
the voxel size increases the spatial resolution but at a cost of computer memory and statistics. There will be less statistics in each voxel if the voxel size decreases. In general, a voxel size of $0.5 \mathrm{~cm}$ in each dimension provides acceptable statistics. Once the voxel size is determined, voxel densities may be assigned either by manual input or through a separate program which assigns densities based on Hounsfield CT numbers. In other words, one can use the CT data set to construct a DMX file that represents the specific patient geometry.

In XVMC particle transport, the material data sources are based on the densities provided within the DMX file. Particles are first transported in water. (density of $\left.1 \mathrm{gcm}^{-3}\right)$. Secondly, the path length, energy losses and scattering angles are reevaluated based on the actual voxel density. Specifically, the path length is scaled by the ratio of unrestricted stopping powers of the material to that of water. This ensures that at the end of each step, the electron in material $x$ has the same energy as an electron transported through one-step of water. These stopping powers are determined from a linear relationship relating physical density and mass stopping power, as shown in ICRU Report 37. The scattering powers are also related to the physical densities. Particles are transported until a cutoff energy or the particle exits the geometry volume. The XVMC output is a dose matrix with the same dimensions as the DMX file. 


\section{CHAPTER 4 \\ Monte Carlo Treatment Planning}

Over the last 10 years, radiotherapy Monte Carlo applications and techniques have dramatically increased. This is partly due to the development of accurate radiation transport software packages (BEAM) and the lower cost of CPU power. Despite available Monte Carlo applications, there are relatively few commercially available MCTP packages. One reason for this could be because there is still a debate as to the clinical justification of Monte Carlo-based patient specific treatment planning. Academic centers often develop their own in-house MCTP systems which integrate with commercial treatment planning systems. These systems are mostly used for patient recalculations and often require a high amount of user and calculation time. There is a need for large scale treatment site-specific analysis of recalculated dose distributions using well-implemented Monte Carlo systems. A system as such could quickly answer the clinical relevance of MCTP. This chapter discuses the issues concerning Monte Carlo treatment planning.

\subsection{Clinical MCTP systems}

Current clinical MCTP systems are summaries in Table 4-1. The main draw back to these systems is the inflexibility in the parameters of the MC simulations. Calculations are preformed in a "black box" environment where parameters including the dose distribution voxel size and variance reduction techniques are not accessible to the user. In general, these systems provide a higher accuracy in dose calculation 
over conventional dose calculation algorithms but none of these systems provide a comprehensive MCTP package with a flexible beam model and patient model to investigate the advantages or disadvantages of MCTP for clinical studies.

Table 4-1: Clinically available Monte Carlo Treatment Planning software.

\begin{tabular}{|c|c|c|}
\hline Manufacture & Processing Power & Description of Features \\
\hline $\begin{array}{l}\text { Nomos } \\
\text { (PEREGRINE) }\end{array}$ & $\begin{array}{l}16 \text { processors } \\
\text { dual }) \text { Pentium } \\
800 \mathrm{MHz}\end{array}$ & $\begin{array}{l}\text { Conformal photon beam and IMRT Monte } \\
\text { Carlo simulations. }\end{array}$ \\
\hline $\begin{array}{l}\text { Nucletron } \\
(\mathrm{VMC}++)\end{array}$ & $\begin{array}{l}\text { Single CPU Pentium } \\
\text { IV XEON, } 2.2 \mathrm{GHz}\end{array}$ & Electron beam Monte Carlo simulations. \\
\hline $\begin{array}{l}\text { Eclipse Varian } \\
\text { (MMC) }\end{array}$ & $\begin{array}{l}\text { Single CPU Pentium } \\
\text { IV XEON, } 2.4 \mathrm{GHz}\end{array}$ & $\begin{array}{l}\text { Electron beam simulations with macro } \\
\text { Monte Carlo. }\end{array}$ \\
\hline
\end{tabular}

\subsection{Developing a MCTP system}

The generic components of a Monte Carlo patient dose calculation system are shown in Figure 4-1. There are two main components that determine the accuracy of the Monte Carlo dose calculation: the radiation source or beam model and the irradiated geometry or tissue interaction coefficient model. A practical system must also include the standard treatment planning tools. These include: (1) file compatibility import / export with regards to standard file formats, (2) beam geometry definition, (3) dose evaluation tools such as dose addition and subtraction and DVH. In addition, the MCTP system needs to be commissioned to ensure the calculations are in agreement with dose measurements. Lastly, the Monte Carlo dose algorithm needs to be calibrated under the same.reference conditions as the treatment machines. This last step normalizes the Monte Carlo dose to the number of monitor units (MUs). 


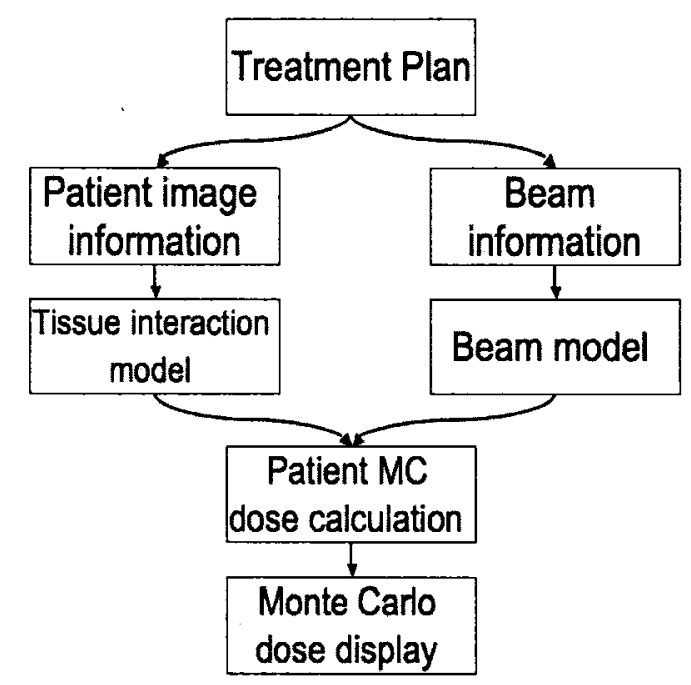

Figure 4-1: Schematic diagram summarizing the generic components of a Monte Carlo treatment planning system [25].

\subsection{Commissioning a Monte Carlo Treatment Planning system}

Monte Carlo simulations provide a computational solution to the radiation transport equation. One must exercise caution while using these techniques to insure the highest level of accuracy. Before these techniques are used in a clinical environment, they must be properly validated. It is good practice to verify the performance of the system with respect to the entire measurement data-set that is available as part of the commissioning process [25]. The various components of beam modeling and patient dose calculation validation are illustrated in Figure 4-2. The validation consists of measuring absolute and relative dose in standard water phantoms as well as heterogeneous phantoms followed by the calculations of dose distributions in water-filled patient geometries. 
MCTP Validation

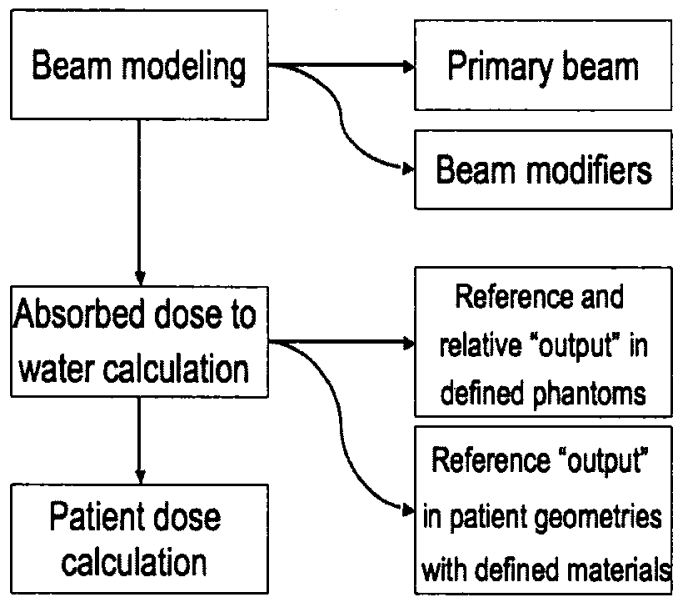

Figure 4-2: Schematic diagram summarizing the validation steps in source or beam model and phantom and patient dose calculations in MCTP [25].

An initial study at McGill University examined the dose distributions in heterogeneous phantoms for clinical electron beams calculated using XVMC and EGSnrc [33]. The dose distributions were extensively compared with accurate measurements. Percent depth dose measurements were preformed with thermoluminescent dosimeters (TLD) within the heterogeneous phantom. The results of the study concluded excellent agreement between the experimental and Monte Carlo dose distributions [33].

A second study at McGill University examined the photon beam depth doses and profiles in a water-phantom with an emphasis on the simulation of the MLC. The results of the study concluded excellent agreement between the experimental and Monte Carlo depth doses and profiles for field sizes defined with the jaws [7]. The MLC model was optimized for leakage and transmission by matching the leakage 
and transmission results from detailed diode detector measurements [7]. This study validated the BEAMnrc model.

A third study at McGill University compared the dose distributions in heterogeneous phantoms calculated from BEAMnrc and XVMC with accurate measurements. Measurements were preformed using TLDs with heterogeneous Solid Water $^{\mathrm{TM}}$ (GAMMEX rmi, Middleton WI) - bone and Solid Water ${ }^{\mathrm{TM}}$ - lung phantoms. Profiles were measured using an electron diode. The study concluded overall the agreement between BEAMnrc and XVMC with measured data was within $2 \%$ except for one case. This work confirms the accuracy of the Monte Carlo models and supports the clinical implementation of Monte Carlo Treatment Planning [18].

\subsection{Normalization of Dose}

For comparison purposes, the dose stated from Monte Carlo calculations should be in the same format, dose per MU, as conventional treatment planning systems. A calibration factor is needed to relate the Monte Carlo dose to the dose per MU. Clinical accelerators at McGill are calibrated to deliver 101 cGy in tissue at the calibration depth, $z_{\max }$ from 100 MUs. While the dose reported by Monte Carlo calculations reports dose per incident particle from the source. The calibration factor is determined by performing a Monte Carlo simulation in water under the clinical calibration conditions. These conditions are; $10 \times 10 \mathrm{~cm}^{2}$ field size, $S S D=100 \mathrm{~cm}$, depth at $z_{\max }$. The Monte Carlo calibration reference dose to water per particle is written as:

$$
\left[\frac{D}{\text { particle }}\right]_{\text {calibration }}=\frac{D_{w}^{M C}\left(10 \times 10, S S D=100, z_{\max }\right)}{\text { particle }}
$$


Finally, the patient dose $\left[\frac{D}{\text { particle }}\right]_{\text {patient }}$ from Monte Carlo calculations can be expressed in terms of absorbed dose per monitor unit as follows:

$$
\frac{D_{\text {patient }}(\mathrm{cGy})}{\mathrm{MU}}=\frac{\left[\frac{D}{\text { particle }}\right]_{\text {patient }}}{\left[\frac{D}{\text { particle }}\right]_{\text {calibration }}} \times \frac{1.01}{1 \mathrm{MU}} .
$$

This equation ignores the known effects of backscattering into the monitor chamber for Varian machines at small field sizes [22] [24]. This influences the output of the accelerator and has to be taken into account when output factors are derived from Monte Carlo simulations. Published studies examined the influence of backscatter to the monitor chamber. One backscatter graph for a $10 \mathrm{MV}$ photon beam is shown in Figure 4-3. It is concluded that these effects are only significant for photon beams with small field sizes [22]. Since patient treatment plans use large MLC-shaped fields, the backscattered monitor chamber fluence can be ignored and Equation 4.2 is valid.

It is important to realize that the calibration dose per particle changes with beam energy. A particle form a $18 \mathrm{MV}$ beam will have a higher dose per incident particle than a particle from a lower energy. Thus, there is a calibration dose per particle for each beam energy. Equation 4.2 then transforms the dose per particle from Monte Carlo into absolute absorbed dose. With the dose distribution in units of absolute dose, one can sum dose distributions from various beam energies.

\subsection{McGill Monte Carlo Treatment Planning}

A block diagram of the McGill MCTP system is shown in Figure 4-4. This system has been implemented and validated at our institution [7]. The first step extracts the beam configurations from the treatment plan to generate a BEAMnrc input file. The simulation produces a phase space file below the last component of 


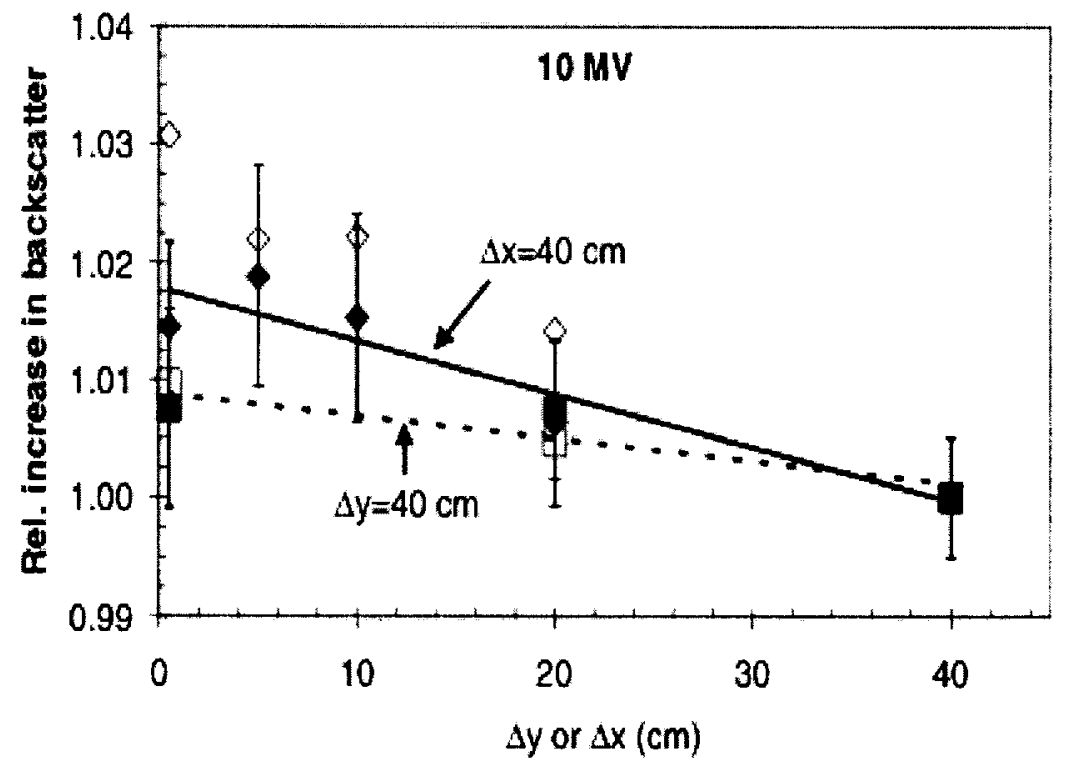

Figure 4-3: The relative increase in backscatter with decreasing field size for rectangular fields, with respect to a $40 \times 40 \mathrm{~cm}^{2}$ field for $10 \mathrm{MV}$ photon beams. Results are given for measurements (filled) and Monte Carlo simulations (open) for lower $X$ jaws (squares) fixed at a $40 \mathrm{~cm}$ field and upper $Y$ jaws (diamonds) fixed at $40 \mathrm{~cm}$ [22]

the BEAM accelerator. The next step is to generate a density matrix from the CT slices. During this step, the CT couch is removed from the CT images as it is not present during treatment. The next step is to run XVMC with the density matrix and phase space file to produce a dose distribution. Finally, the dose distribution is normalized to the number of monitor units and displayed.

The McGill MCTP system is currently used to recalculate patient dose distributions. The system has been validated and it's accuracy proven. Although, the steps involved in this system require a considerable amount of manual input. There have been many small programs written to speed up ease the process but there is still a 


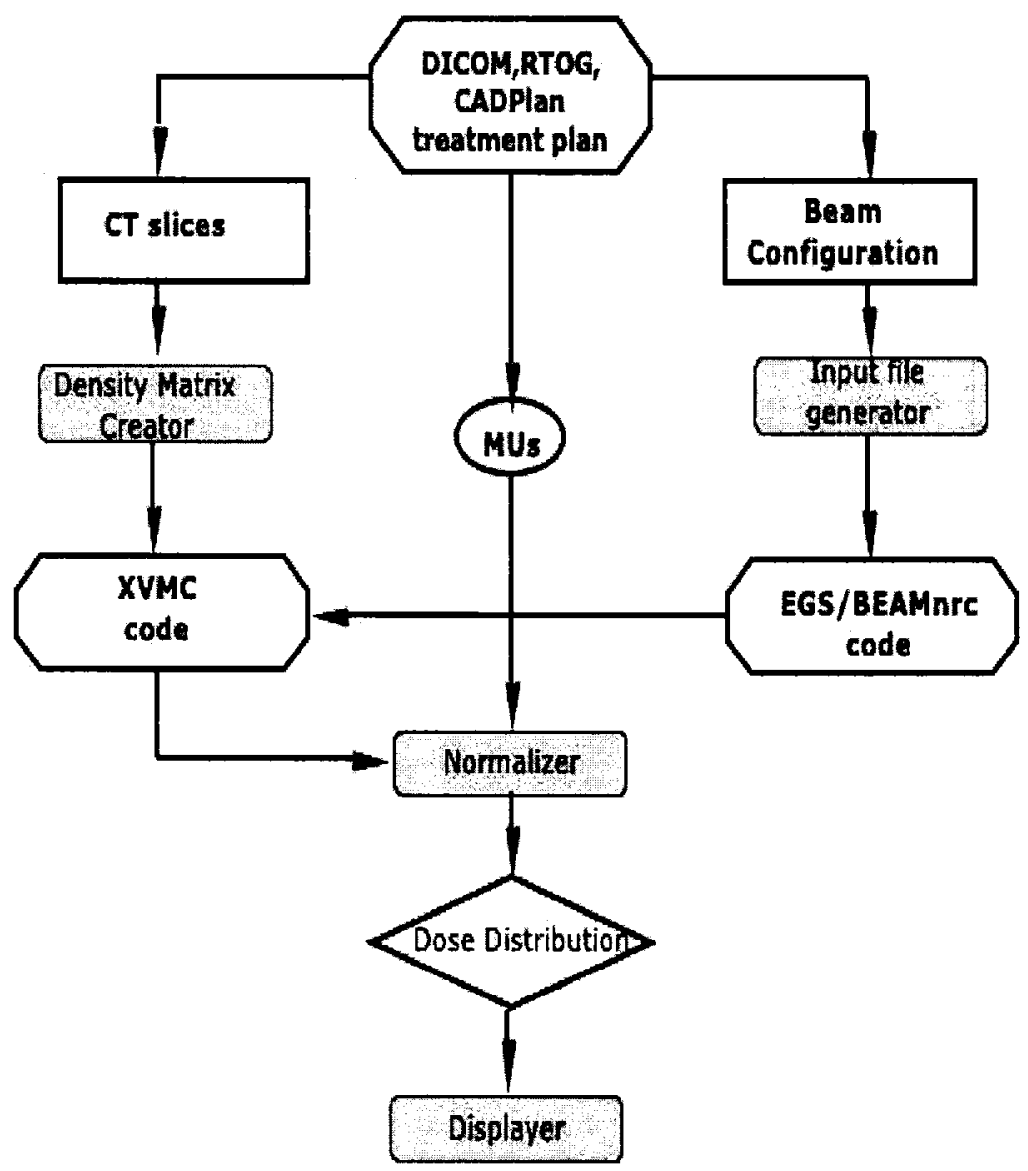

Figure 4-4: Block diagram of the steps involved for McGill Monte Carlo treatment planning system [7].

need for an automated process to quickly produce Monte Carlo dose calculations. One solution would be to develop a GUI that lets the user control all the simulation properties. The GUI must also control the simulation process and have display capabilities to display the calculated dose distribution. A GUI of this type has been developed at McGill and is described in the following chapter. 


\section{CHAPTER 5 \\ MMCTP GUI for Monte Carlo Treatment Planning}

\subsection{Introduction}

Radiotherapy treatment planning relies on the accuracy of dose calculations and other parameters to generate a patient treatment plan. Radiotherapy deliveries utilize the information provided by the treatment planning system and it is generally accepted that clinical outcome can be improved if accuracy in the dose delivery is further improved [10]. Proven Monte Carlo calculations increase the planning accuracy however; Monte Carlo radiotherapy treatment planning (MCTP) has only now made a slow entry in the clinical environment, taking considerably longer than envisaged ten years ago [20]. Reasons for this are:

a Difficulties in beam model commissioning

b Duration of plan calculation

c Clinical issues related to the interpretation of the treatment plan

$\mathrm{d}$ Whether photon beam MCTP is advantageous given the accuracy of well commissioned convolution algorithms

For further research and to establish large-scale retrospective studies, there is a need for a software platform that combines different forms of treatment planning and analysis tools with ease. Current software environments that share these goals include CERR [29] and MINERVA [28]. However, these both require additional (commercial) packages installed, use interpreted and slow language tools or are not 
easily available. The McGill Monte Carlo treatment planning system, MMCTP, provides a flexible software environment to integrate Monte Carlo planning with current new treatment modalities and deliveries. The aim of this project is to extend patient specific radiotheapy treatment planning with:

- Monte Carlo treatment planning (external beam electron and photon planning)

- The use of multi modality and multi instance imaging

- Analysis tools for plan evaluation and studies of outcome correlations

The MMCTP platform was designed using REALbasic (Real Software Incorpo-

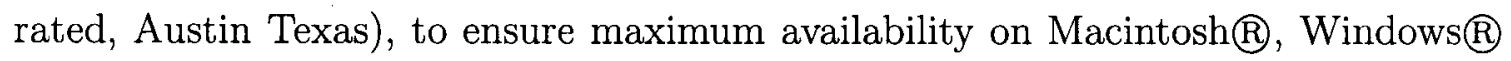
and Linux operating systems. Flexible imports (RTOG, DICOM_RT, and CADPlan CART formats) allow broad base data sources. The MMCTP design consists of a GUI, which runs on a simple workstation connected through standard secure-shell protocols to a cluster for lengthy Monte Carlo calculations. The local station controls the shell. This strategy enables the use of an off-site cluster that does not require specific software (i.e. daemons, etc) installed. In addition, the design allows for anonymous patient information on the calculation cluster and a minimal amount of data interchanged between the workstation and calculation cluster. The impact of this tool lies in the fact that it allows for systematic, platform independent multicentre large-scale Monte Carlo planning calculations for different treatment sites for retrospective and prospective studies. The current features included within MMCTP are:

- RTOG, DICOM_RT and CADPlan radiotherapy format: CT scans, contours, beam geometry, dose distributions 
- Axial/Sagittal/Coronal slice viewing for CT image, contours and dose distributions

- Contouring tools

- Colour-wash and isodose line display

- BEAMnrc and XVMC simulations for photon and electron beams

- DVH analysis and dose matrix comparison tools

\subsection{Philosophy and Design Decisions}

MMCTP was designed as a software environment for the research development of patient specific treatment planning with Monte Carlo as the dose engine. The basic philosophy behind MMCTP includes a workstation for treatment planning tools and a powerful, possibly remote cluster for Monte Carlo dose calculations. The programming goals for a practical application included:

- Multi-Platform application (Macintosh $\AA$, Windows $\AA$, Linux)

- Low hardware demand for workstation

- Easy cluster connection (sftp, telnet, ssh)

- Minimal network traffic

- Anonymous patient information on calculation cluster

- Flexible imports (RTOG, DICOM_RT, etc)

A multi platform application combined with low hardware demand enables anyone to use MMCTP. MMCTP connects to a potentially remote cluster but specific patient information is never transferred to the cluster, ensuring patient confidentiality. The import formats, RTOG and DICOM_RT, are both standard formats for storing electronic medical data. DICOM_RT radiotherapy data includes CT and or 
MRI image sets, radiotherapy plan information, and dose distributions. After exporting radiotherapy data from a commercial treatment planning system in either RTOG or DICOM_RT format, MMCTP can import the data.

\subsubsection{Software selection}

The MMCTP GUI was built using REALbasic because of its cross-platform flexibility and low hardware demand. With built in 3D graphic tools, REALbasic allows the possibility of surface rendering and 3D room's eye view. It is a rapid application development environment (RAD) meaning one can quickly design GUIs. In addition, it is full fledge Object Oriented Programming (OOP) offering great flexibility for programming changes. Arguably its best feature for MMCTP is its ability to make shell calls to other programs written in any language.

\subsection{MMCTP Programming}

The initial programming task was to develop an organized object class structure to store treatment planning information within memory. The RTOG format, a welldocumented and widely available radiotherapy archiving mechanism, was used as a model to base the REALbasic objects. This not only helps to organize the variables but also ensures native reading and writing to RTOG. In addition, new MMCTP programmers will benefit from a simple RTOG object based GUI.

While the RTOG format provides a convenient object class, it does not provide a flexible file storage format. A new file format called McGill RT was designed for saving patient plans on the workstation. The file system resembles the RTOG format but includes efficiency improvements. The RTOG format was designed for exporting and importing patient plans. Therefore, there is no simple method for saving small 
changes. Within the RTOG format, save changes require a complete rewrite of all patient data. The McGill RT format was developed to minimize redundant information and minimize the number of files edited for save changes. Figure 5-1 shows a schematic diagram of McGill RT file format. The format uses a combination of folders and files which are either binary or text. Images and dose distributions are stored as binary files while structures and beam information is stored as a text file. The file structure of the McGill RT format contains a main McGill folder, under which lie folders for each patient. The patient folders are titled with the patient name and ID number. Within a patient's folder there can be multiple image sets (CT, MRI, ultrasound (US)) each set with it's own folder. Under each image set, there are folders for multiple plans as well as a folder for the images and structures called McGill RT. The plan folders store beams, doses, and Monte Carlo control files. The file storage format is flexible enough to allow for future adaptation to include image data sets such as, image fusion between MRI, PET, CT and time phased computed tomography (4D CT).

\subsubsection{McGill RT Format}

\section{Image files}

Images are stored individually under the McGill RT folder. The image files are named sequentially with the file type as.$i m g$. The files are binary, containing a slice position and the pixel data. In order to properly read these files, there is an image properties text file called RT.dir as shown in Figure 5-2.

The properties file stores properties for reading and displaying the images. The dimension size and the bytes per pixel are essential for correctly reading a binary 


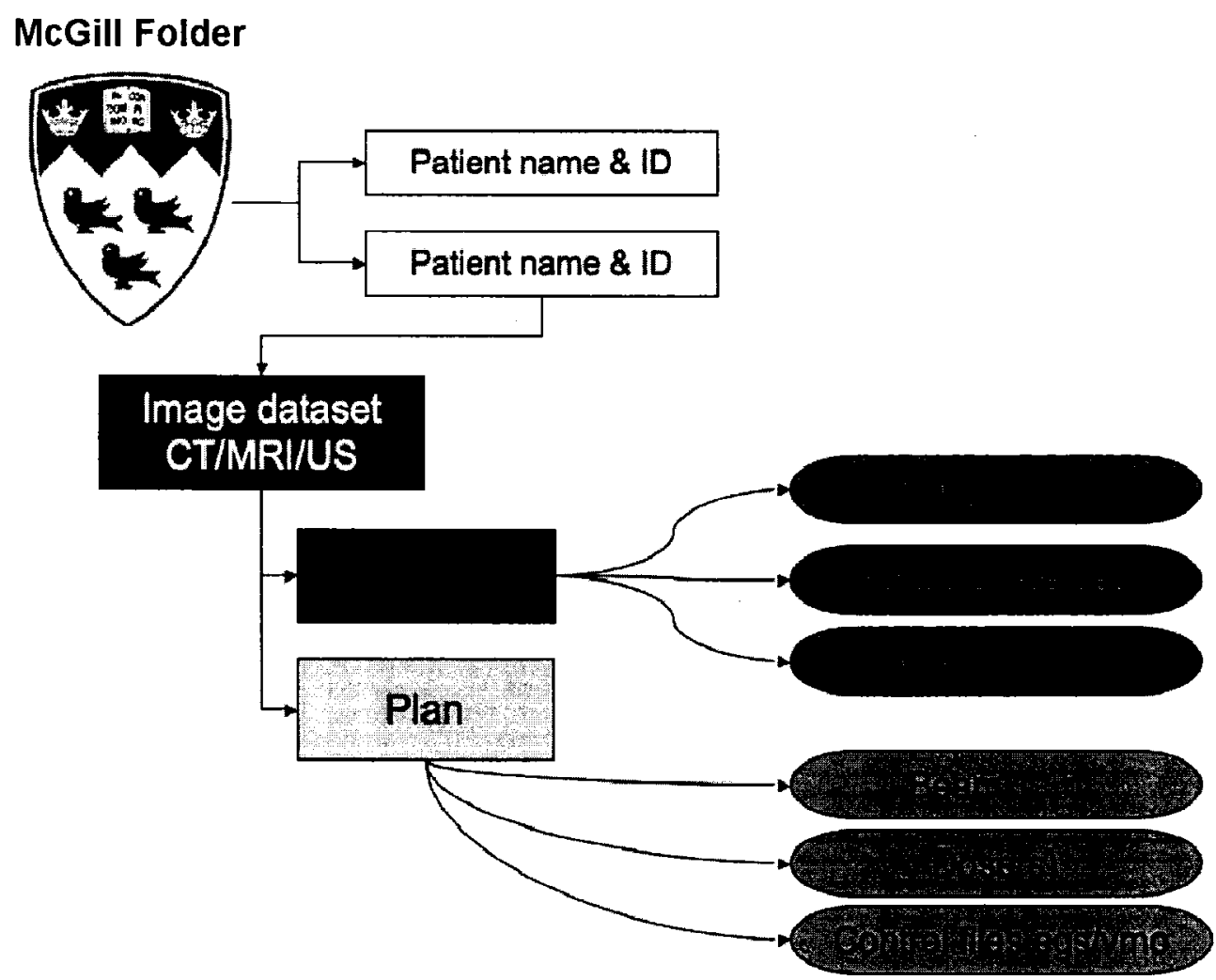

Figure 5-1: McGill RT file structure, folders and files 


\begin{tabular}{ll}
\hline \hline Patient Name & $:=$ (string) \\
Patient ID & $:=$ (integer) \\
Image type & $:=($ CT,MRI,US) \\
CT offset & $:=$ (integer) \\
Grid unit width & $:=($ cm per pixel) \\
Grid unit height & $:=$ (cm per pixel) \\
Number representation & $:=$ (character) \\
Bytes per pixel & $:=($ integer $)$ \\
Size of Dimensions 1 & $:=($ integer $)$ \\
Size of Dimensions 2 & $:=($ integer $)$ \\
X offset & $:=(\mathrm{cm})$ \\
Y offset & $:=(\mathrm{cm})$ \\
Slice thickness & $:=(\mathrm{cm})$ \\
\hline \hline
\end{tabular}

Figure 5-2: McGill RT image properties file: properties and their variable type.

image file. Generally, the $(X, Y)$ origin of the patient coordinate system $5-3$ is at the exact centre of the image. The $X$ and $Y$ offsets, stated in $\mathrm{cm}$, permit a displacement from the image centre.

In order to properly visualize the image, the grid units are used to adjust the image dimension. These units relate the pixels to a physical dimension for proper visualization scaling. The grid unit states the width and height in $\mathrm{cm}$ of one pixel. The image type labels the images as CT, MRI, US or PET images. Currently, MMCTP can only open CT images. Additional parameter are required for the future implementation of 4D data sets.

After reading the properties file, MMCTP has all the information required to read in each .img file. Within each image file, the first 4 bytes represent the $Z$ image position followed by the pixel data. The pixel data is read in row by row with the first pixel corresponding to the top left corner of the image. 


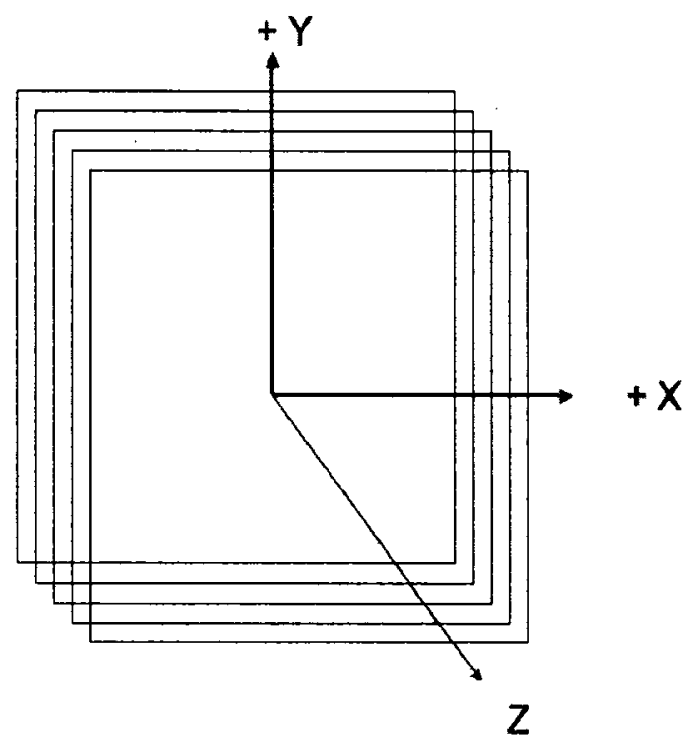

Figure 5-3: McGill RT patient coordinate system: The $(X, Y)$ origin is located at image centre with positive $X, Y$ directions as indicated. The $Z$ axis does not have a specific positive direction.

\section{Structure files}

The structures are a sequence of three-dimensional coordinates which define a volume of interest. A volume can include target volumes and organs at risk. The coordinates are grouped together in planes, which coincide with planes on the CT image slices. A given structure does not have to be defined on all planes. Within a given plane, a structure may consist of one or more segments. Where each segment is a sequence of at least four points which define a closed curve on the surface of the volume. The structure files are text files named sequentially with the file type struct. These files are found under the McGill RT folder because they are associated to the image files. Each structure file contains a header section followed by the structure coordinates. The specific format of these files is shown in Figure 5-4. 


\begin{tabular}{|c|c|}
\hline STRUCTURE NAME & := PTV2 \\
\hline NUMBER REPRESENTATION & $:=$ CHARACTER \\
\hline STRUCTURE FORMAT & $:=$ SCAN-BASED \\
\hline STRUCTURE COLOUR RGB & $:=252 / 18 / 41$ \\
\hline NUMBER OF SCANS & $:=3$ \\
\hline "NUMBER OF LEVELS" 3 & (total number of scans) \\
\hline "SCAN NUMBER" 1 & (=1 for first scan, etc) \\
\hline "\# OF SEGMENTS" 0 & (number of segments in this level/scan) \\
\hline "SCAN NUMBER" 2 & \\
\hline "\# OF SEGMENTS" 1 & \\
\hline "\# OF POINTS" 4 & (number of points in first segment) \\
\hline $0,1.459,20.86305$ & ( $X, Y, Z$ coordinates of each point) \\
\hline$-0.56,1.387,20.86305$ & \\
\hline$-0.776,1.387,20.86305$ & \\
\hline$-0.848,1.315,20.86305$ & \\
\hline "SCAN NUMBER" 3 & \\
\hline "\# OF SEGMENTS" 0 & \\
\hline
\end{tabular}

Figure 5-4: McGill RT structure file: Example structure file, header information followed by struture points.

The header information contains the structure name, colour and number of scans. The number representation and format are always character and scan-based. The structure points follow the header information. The structure points are arranged in order per scan number. Each scan number is listed followed by the number of segments per scan. If there exits a segment, the next line reports the number of points, and the subsequent lines report the points. The coordinate points are in centimeters relative to the patient coordinate system.

\section{Plans}

Patient plans are assigned folders within an image set folder. The files found within a patient plan folder include: 
- Beam files

- Dose distribution files

- Monte Carlo control files

The following sections describe these files.

\section{Beam Geometry files}

A beam geometry file contains the information defining an external radiation beam. These include various properties such as: treatment unit, beam energy, beam applicators, number of MUs, number of fractions, aperture type, wedge angle and orientation, collimator gantry and couch angle, isocentre distance, isocentre coordinates $(x, y, z)$, and $X, Y$ jaw positions.

The MLC leaf positions are not included within the beam file. Instead, there is a MLC file which contains the MLC fields for all beams of a plan. Each beam has it's own beam geometry file with .Beam as the file type. The specific format of these files is shown in Figure 5-5.

\section{Dose Distribution files}

The dose distribution is a matrix of dose values at one or more points throughout a patient. Typically, the distribution is calculated on a three dimensional grid. The format allows for a regular spaced grid, one in which a two dimensional array of points is defined on one or more evenly spaced parallel planes. This format permits the computation of dose on a two-dimensional array of points on each CT scan.

The coordinate system for the array of dose points is defined with the patient coordinate system. Within one plane, a two-dimensional array of points is defined with the $x, y$ position of the top left hand corner point, the number of dimensions in 


\begin{tabular}{|c|c|}
\hline$\overline{\mathrm{BEAM}} \#$ & $:=($ beam number in plan $)$ \\
\hline TREATMENT UNIT & $:=($ treatment unit name ex CL21EXA $)$ \\
\hline BEAM MODALITY & $:=($ electron, photon $)$ \\
\hline BEAM ENERGY & $:=($ energy ex $18 \mathrm{MV})$ \\
\hline BEAM APPLICATOR & $:=($ for electrons only $)$ \\
\hline BEAM DESCRIPTION & $:=($ text description of beam $)$ \\
\hline RX DOSE PER TX (GY) & $:=($ fraction dose $)$ \\
\hline MUs & $:=($ number of MUs $)$ \\
\hline NUMBER OF TX & $:=$ (number of fractions) \\
\hline FRACTION GROUP ID & $:=($ id to group beams of common fraction $)$ \\
\hline BEAM TYPE & $:=($ static or arc $)$ \\
\hline COLLIMATOR TYPE & $:=$ (symmetric or asymmetric) \\
\hline APERTURE TYPE & $:=($ block or MLC $)$ \\
\hline WEDGE ANGLE & $:=($ wedge angle in degrees ex 15$)$ \\
\hline WEDGE ORIENTATION & $:=($ wedge orientation ex in,out,left,right $)$ \\
\hline WEDGE DYNAMIC & $:=($ dynamic wedge boolean ex true/false $)$ \\
\hline COLLIMATOR ANGLE & $:=$ (angle in degrees) \\
\hline GANTRY ANGLE & $:=$ (angle in degrees) \\
\hline COUCH ANGLE & $:=$ (angle in degrees) \\
\hline NOMINAL ISOCENTER DIST & $:=($ isocentre distance in $\mathrm{cm})$ \\
\hline NUMBER REPRESENTATION & $:=$ (character) \\
\hline PLAN ID OF ORIGIN & $:=($ plan ID of beam for grouping beams $)$ \\
\hline "Isocentre coordinate"- $-6.3,-2.5,-33.52$ & (isocentre $\mathrm{x}, \mathrm{y}, \mathrm{z}$ in $\mathrm{cm}$ ) \\
\hline "Collimator Setting X" 6.2, 6.2 & (collimator $\mathrm{x} 1$ and $\mathrm{x} 2$ positions in $\mathrm{cm}$ ) \\
\hline "Collimator Setting Y" 7.8, 7.8 & (collimator $\mathrm{y} 1$ and $\mathrm{y} 2$ positions in $\mathrm{cm}$ ) \\
\hline
\end{tabular}

Figure 5-5: McGill RT beam geometry file 


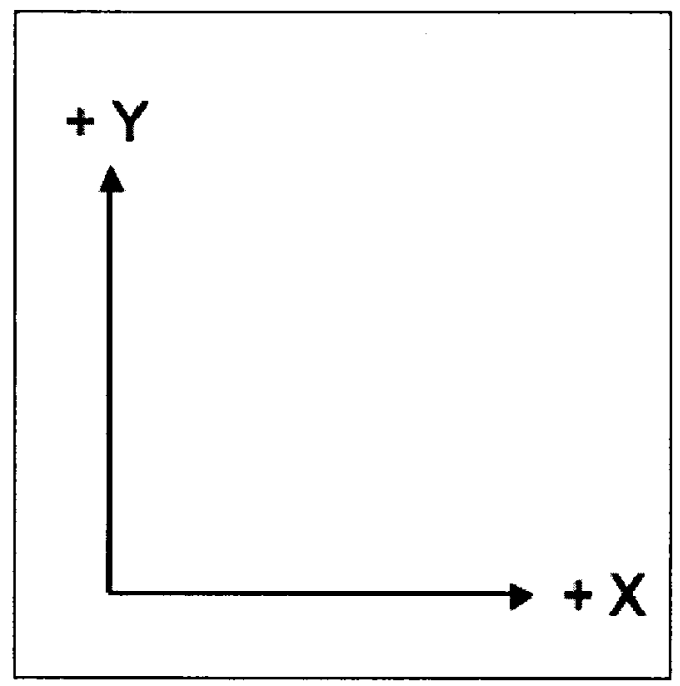

Figure 5-6: McGill RT axial dose orientation

$x, y$ and the grid spacing in $x, y$. Each axial plane is identified with its $Z$ position, which normally corresponds to that of the axial CT image. The dose data is typically in units of Grays.

The dose files are written in binary format with extension .dose. Each file contains a header block followed by the dose distribution matrix. The header contains the properties of the dose distribution, which are written as single values. These properties are shown in Figure 5-7. The dose distribution begins at the 100th byte. The dose values are written as single values per axial plane. The $Z$ position, written as a double, precedes the distribution for each plane.

\section{Monte Carlo control files}

Aside from the input files required to run BEAM and VMC, there is an additional file created called $M C_{-}$Settings to track the Monte Carlo process for all beams of a plan. An example of this file is shown in Figure 5-8. The file is separated 


\begin{tabular}{ll}
\hline \hline Coordinate X 1st point & $:=(\mathrm{cm})$ \\
Coordinate Y 1st point & $:=(\mathrm{cm})$ \\
Coordinate Z 1st point & $:=(\mathrm{cm})$ \\
Size of Dimension 1 & $:=(\#$ horizontal points $)$ \\
Size of Dimension 2 & $:=(\#$ vertical points $)$ \\
Size of Dimension 3 & $:=(\#$ planes $)$ \\
Horizontal Grid & $:=(\mathrm{cm}>0)$ \\
Vertical Grid & $:=(\mathrm{cm}<0)$ \\
Dmax & $:=(\mathrm{Gy})$ \\
\hline \hline
\end{tabular}

Figure 5-7: McGill RT dose header block

into two parts, dealing with EGS BEAM and VMC. The BEAM section include the job split number, number of particles, and the simulation status. The job split number has no effect on the simulation result but will greatly affect the simulation time. Computer clusters split jobs to multiple processors for quick calculations. The split number is the number of processors utilized for one simulation. The number of particles is the number of initial particles from the source. The simulation status stores the percent progress of each beam simulation. A progress value of -1 means the simulation has not started.

The VMC section is split into two parts. The first part states the DMX (section 3.5) settings, and the second part is specific to the individual beams. The DMX settings can change from plan to plan, as there are a few ways to generate the DMX file. These settings store a record of how the DMX was generated. The 'VMC Properties' section lists all images and their DMX generation option. The option value can be $(-1,0,1,2)$ depending on whether or not the image is replaced by another image, skipped or used, during the DMX generation. The 'VMC Structure Density' section lists all structures, their density, their density boolean, and their fill 
order. If the user decides to use the structure densities, the fill order will determine which densities are painted first. This only affects the DMX if two or more structures overlap each other. The 'VMC Simulation Progress' records the XVMC dose used by each beam and the progress of the simulation. The last line of this file records the dose to water conversion value.

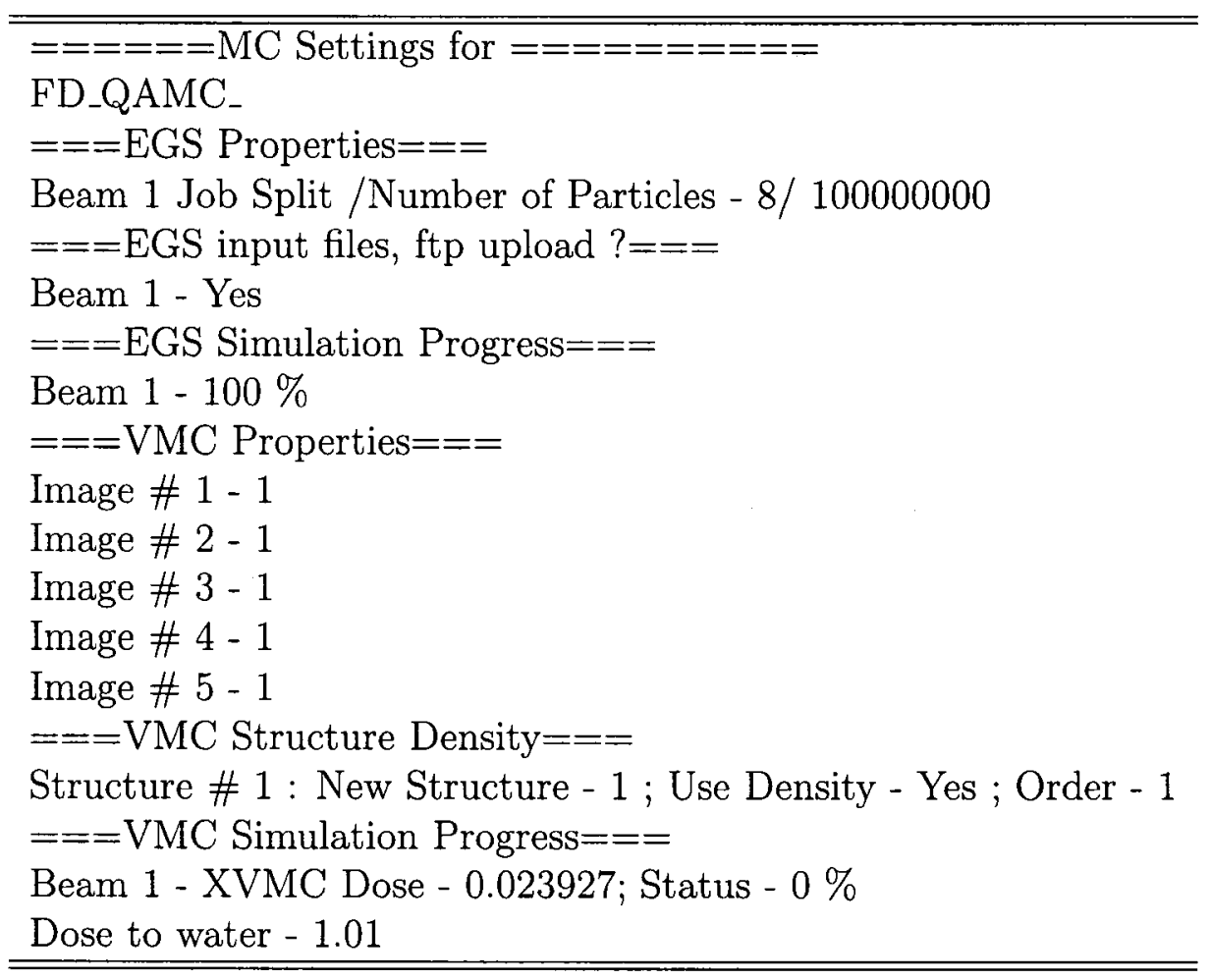

Figure 5-8: McGill RT Monte Carlo settings file

\subsubsection{Monte Carlo Treatment Planning}

As mentioned in Section 3 there is a selection of codes to choose from for Monte Carlo simulations. The general programing philosophy is to make an interface to any Monte Carlo engine. Initially, MMCTP will use EGS BEAMnrc [16] for modeling radiotherapy sources and XVMC [30] for in patient transport. This is a two step 
process which requires separate input files and control logic. BEAM models the radiation transport within the linac head based on the beam geometry properties to produce a phase space file at $70 \mathrm{~cm}$ from the source. Upon completion of BEAM, VMC transports the phase space file through the patient geometry which is based on the CT images and defined structures. Once complete, VMC writes a binary dose .$d 3 d$ file. MMCTP downloads this file to the workstation to produce a VMC dose distribution in McGill RT format. A schematic diagram of the MMCTP data flow is shown in Figure 5-9.

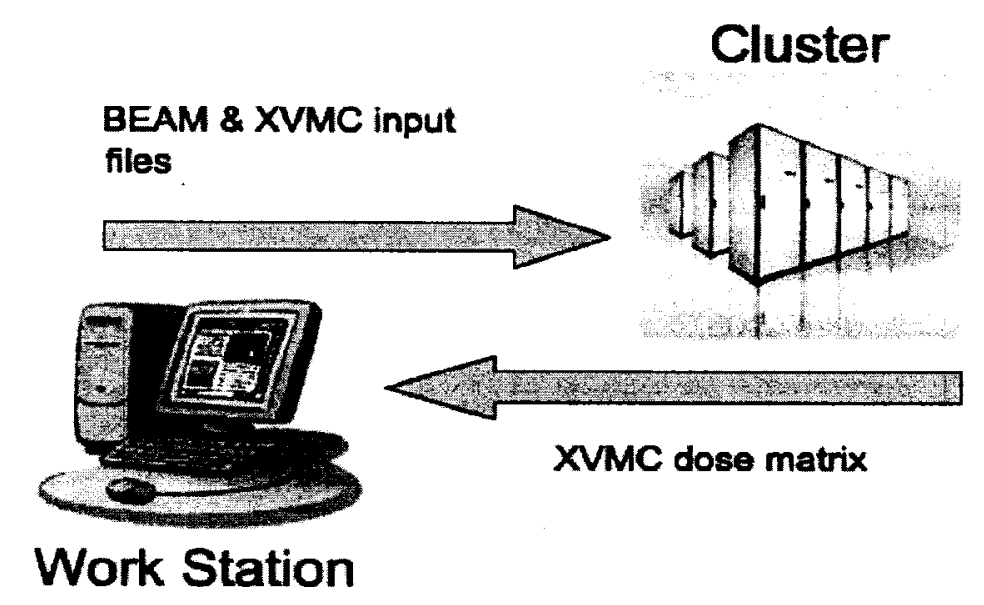

Figure 5-9: MMCTP data flow, input files to cluster and $d 3 d$ file to work station 


\subsection{MMCTP Modules}

The MMCTP program was split into four main modules to simplify the programing task. These modules include:

- Import/Conversion

- Treatment Planning

- Monte Carlo

- Contour Editor

\subsubsection{Import/Conversion}

MMCTP uses only McGill RT format for reading and saving data. The import conversion module reads patient information from standard formats (DICOM_RT, RTOG, CADPlan) and rewrites this information into McGill RT format. Converting patient information to McGill RT format is the first step in using MMCTP.

\subsubsection{Treatment Planning}

Treatment planning is a broad term that groups together different aspects of plan design, treatment planning process, and radiation delivery strategy. Treatment planing requires certain fundamental tools for creating acceptable plans. The planning module groups these tools together within one interface. Current tools of MMCTP include external beam editing, image visualization options, and dose analysis tools.

\section{External Beam Editing}

Beam editing allows the user to edit the beam properties as described in $5-5$. Easy access of these properties is necessary for an efficient software package. These properties are often varied to optimize the dose distribution of a plan. 


\section{Image Visualization}

Visualization tools are an essential component to treatment planning. These tools help identify patient anatomy and are used for qualitative assessment of dose distributions. The image visualization tools include:

- CT image display (axial / sagittal / coronal)

- image window and level settings

- zoom options

- image panning

- transparency settings for structure contours and colour-wash dose distributions

- isodose settings

- cross-hairs for identifying the sagittal and coronal positions

So far, these tools are applied in two dimensions. Extending this to three dimensions adds additional benefits in beam editing and dose visualization. With three dimensions, one can superimpose the beam geometry with the patient's geometry to visualize the beam field throughout the patient. MMCTP includes a 3D beam's eye view (BEV) window which transports $2 \mathrm{D}$ objects into 3D vector objects for lifelike visualization.

\section{Dose Analysis Tools}

Analyzing and quantifying the dose distribution is the most important aspect of treatment planning. The dose analysis tools help quantify the dose distribution with DVH graphs and dose matrix operations. The DVH graphs are calculated by interpolating the dose distribution onto the $\mathrm{CT}$ resolution. The accuracy of this calculation is extremely important. 
The matrix operations are used to add, subtract, multiply or divide dose distributions with other dose distributions. This provides a convenient method for summing the dose distributions from multiple plans.

\subsubsection{Monte Carlo}

The Monte Carlo control module handles all events for Monte Carlo calculations. Since MMCTP uses a two-step process to generate Monte Carlo dose distributions, the Monte Carlo module controls BEAM for phase space generation and VMC for patient-specific dose calculations. In order to run these calculations, input files are prepared from the beam geometry and uploaded to the cluster using the REALbasic shell commands. Following this, a command runs the calculation. For each beam, the BEAM input file is pieced together from template input files stored on the workstation. The specific beam geometry properties dictate which template files to use.

The VMC input file is generated from one template file and a few beam geometry properties. Before running VMC, the module uploads the patient-specific DMX generated from either the CT Hounsfield numbers or from the structure contours and their assigned densities or a combination of them. There also exists an option to remove the CT couch from the DMX since it is not present during treatment.

The MMCTP shell is in constant communication with the cluster, most often checking the progress of calculations or running codes to combine phase spaces files. Upon completion of VMC, the workstation downloads the . $d 3 d$ file for each beam. The last step combines the.$d 3 d$ files from all beams to produce a local McGill RT dose distribution file. This step requires the dose to water correction, VMC 
calibration dose per beam and the number of monitor units per beam to calculate a dose distribution in Gy.

Other properties which influence the simulation and are not part of the beam geometry include:

- number of initial particles

- dose to water correction

- VMC calibration dose

\subsubsection{Contour Editor}

In radiotherapy, the contours are drawn by an oncologist prior to the treatment planning process. After importing these contours into MMCTP, the contour editor allows the option of editing existing contours as well as adding new contours. For example, bolus may be added as a new contour. It is important to include the bolus contour because it lies outside the external contour of the patient and thus will change the DMX file. Contours are associated with axial CT slices and thus do not change from plan to plan. The editor is a separate window with many editing features. These editing features include:

- add point

- move point

- delete point

- scale segment

- move segment

- copy segment to superior slice

- copy segment to inferior slice 


\section{CHAPTER 6 \\ MMCTP Results and Validation}

\subsection{MMCTP GUI}

The MMCTP GUI is a powerful research platform for the development of photon and electron external beam Monte Carlo treatment planning. MMCTP incorporates all the basics functionalities of modern treatment planning software coupled with extensive dose comparison and manipulation tools. This section describes the MMCTP programming results and the second half of this section summarizes MMCTP testing, measurement and patient recalculation results.

\subsubsection{Configurations folder}

The configuration folder stores all MMCTP system files. These files include a system preference file, accelerator property file, DICOM dictionary file, electron cutout files, VMC dose calibration file, and many Monte Carlo input files.

\section{System Preference file}

The system preference file is a basic text file named pref.txt which stores the path location of four folders. This set includes import paths for RTOG, DICOM, CADPlan folders as well as the McGill folder. The preference file is used to determine the location of folders for reading and writing data.

\section{Linac Property file}

-The linac file, named mmctp.configuration, defines the available linac models in terms of energy, radiation type, applicator options and MLC type. This file also 
includes a section for the physical wedges. The file is used to specify beam properties for specific linac models. The file format is defined in Figure 6-1.

\section{DICOM dictionary file}

The DICOM dictionary file named DICOM_dict.txt provides a reference lookup for the DICOM reader. This file is required in-order to read DICOM files.

\section{VMC dose calibration file}

The VMC calibration factor relates the Monte Carlo output to the dose per MU. The calibration file named XVMC_dose.txt, stores a calibration factor for all linac energies. This file contains a list of linacs, their energies and the calibration factor. The file also contains the dose to water correction factor.

\section{BEAM input files}

There are many BEAM input files within the configurations folder. These files are used to construct the specific BEAM input file based on the beam geometry properties. For each linac energy, there is a base template file. MMCTP edits these files to create a BEAM input file. The template files store specific information related to the linac that does not change from run to run. This includes, initial electron energy distribution, the dimensions and materials of all parts of the machine, the location of output planes, how to track a particle's history, which variance reduction technique to apply, what transport parameters to use, etc. Aside from these, there are four parameters that do change from run to run. Parameters specific to the beam geometry properties include the jaw settings, MLC field, and wedge component. A fourth parameter specifies the initial number of particles for the simulation. An example of the jaw component module is shown in Figure 6-3. 


\begin{tabular}{llll}
\hline \hline *Available electron accelerator models: \\
& & & \\
CL18, & $9 \mathrm{MeV}$, & $4 \times 4$, & $0 \mathrm{MLC}$ \\
CL18, & $9 \mathrm{MeV}, \quad 6 \times 6$, & $0 \mathrm{MLC}$ \\
CL18, & $9 \mathrm{MeV}, \quad 8 \times 8$, & $0 \mathrm{MLC}$ \\
CL18, & $9 \mathrm{MeV}, \quad 10 \times 10$, & $0 \mathrm{MLC}$ \\
CL18, & $9 \mathrm{MeV}, \quad 15 \times 15$, & $0 \mathrm{MLC}$ \\
CL18, & $12 \mathrm{MeV}, \quad 4 \times 4$, & $0 \mathrm{MLC}$ \\
CL18, & $12 \mathrm{MeV}, \quad 6 \times 6$, & $0 \mathrm{MLC}$ \\
CL18, & $12 \mathrm{MeV}, \quad 8 \times 8$, & $0 \mathrm{MLC}$
\end{tabular}

*Available photon accelerator models:

CL21EXA, $6 \mathrm{MV}, \quad 120 \mathrm{MLC}$ CL21EXA, $18 \mathrm{MV}, \quad 120 \mathrm{MLC}$

*Available Wedges:

\begin{tabular}{lllll} 
15, & Right, & Left, & In, & Out \\
30, & Right, & Left, & In, & Out \\
45, & Right, & Left, & In, & Out \\
60, & Right, & Left, & In, & Out \\
\hline
\end{tabular}

Figure 6-1: mmctp.configuration: There are 3 sections to this file, electron, photon, and wedges. The electron and photon section has one line for each linac configuration. The linac name is first, followed by the energy, applicator for electrons, and MLC. The wedges are defined with the wedge angle and the wedge orientation options. 


\begin{tabular}{lll}
\hline \hline CL21EXA, & $6 \mathrm{MV}$, & 0.023927 \\
CL21EXA, & $18 \mathrm{MV}$, & 0.164814 \\
Dose to water, & 1.01 & \\
\hline \hline
\end{tabular}

Figure 6-2: XVMC_dose.txt: The linac name is first, followed by the energy and the VMC dose per particle at the calibration point. The last line in the file is the correction for dose to water from dose to tissue.

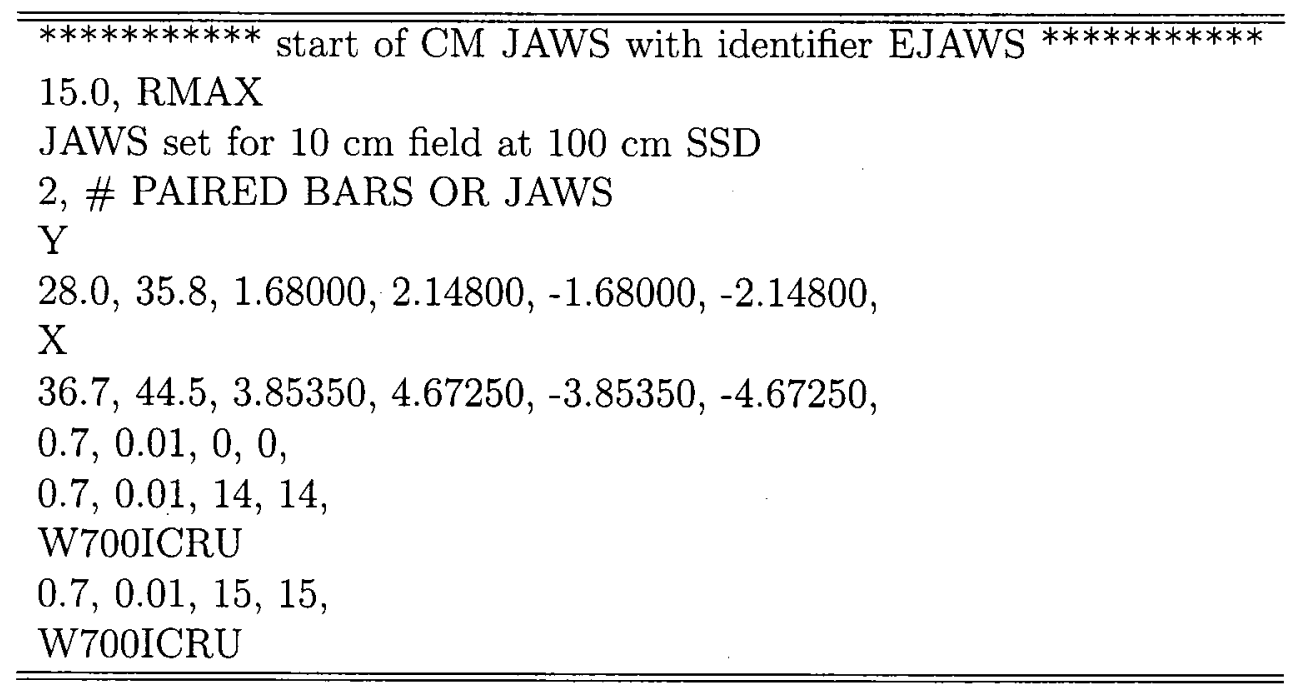

Figure 6-3: BEAM input file, jaw component module. $X, Y$ jaw positions

A second group of input files includes the wedge component module files. These files specify individual wedge angles and orientations. MMCTP uses one wedge file to generate the wedge component module of the BEAM input file. An example of a wedge file is shown in Figure 6-4

\section{VMC input file}

There is one VMC template input file called input.vmc as shown in Figure 6-5. Every VMC simulation uses this file as a base to create the beam specific input file. The parameters that remain constant from run to run include the random number set, beam weight, number of particles to transport and phase space $S A D$ location. 


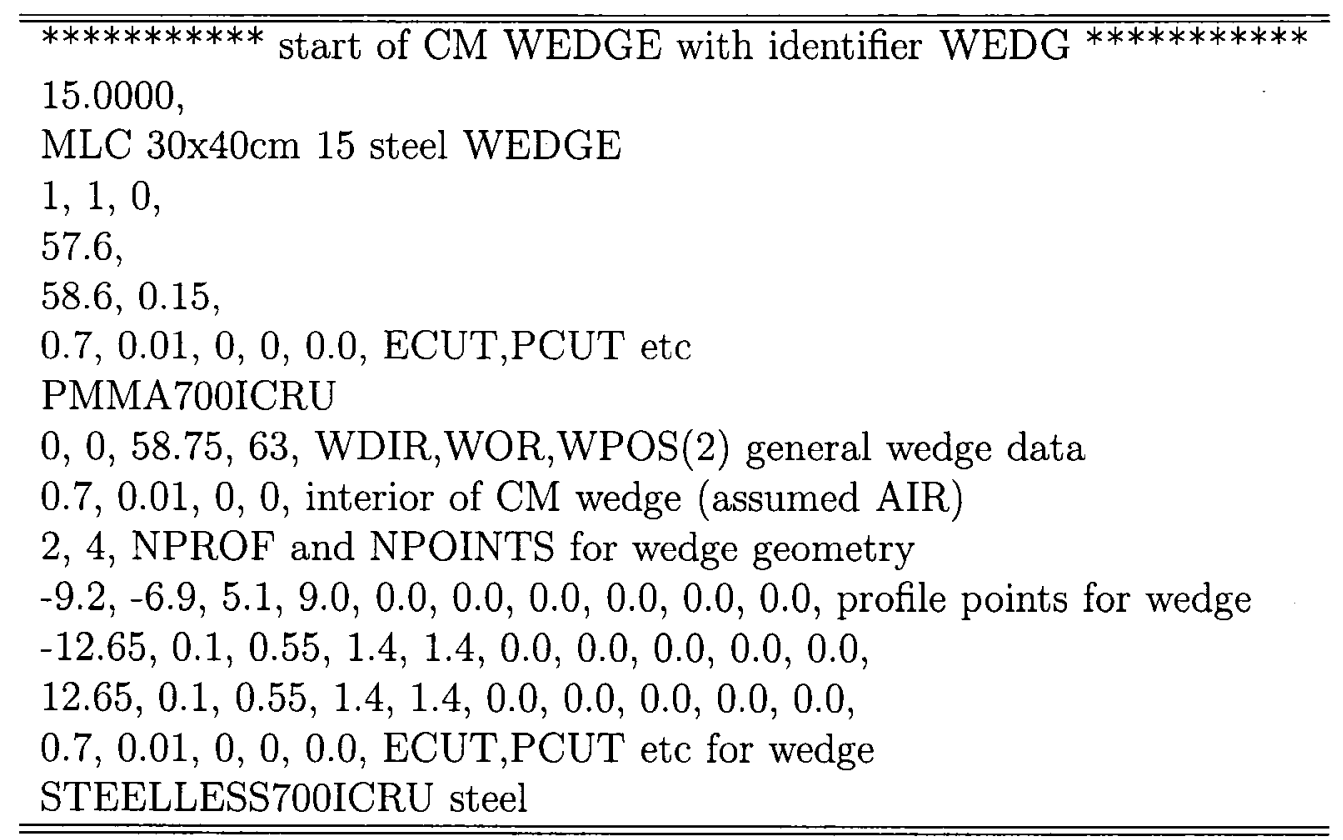

Figure 6-4: BEAM input file, wedge component module for a 15 degree wedge

\subsubsection{Import Window}

The import window allows the user to transfer patient data into McGill RT format. A pulldown menu selects the importing format which can be RTOG, DICOM or CADPlan. Once the format is selected, the program will scan the specific folder associated to that format and populate the import patient list-box with patient information. The import folder path may be changed by clicking on the path and choosing a new folder. This change is then saved in the preference file. Once the patient is displayed in the import list-box, the user must select the patient and press the transfer button. The program will then read in the patient information and rewrite in McGill RT format. Upon completion, the patient will be listed in the McGill list-box. 


\begin{tabular}{ll}
\hline \hline *GLOBAL-DATA & - \\
-WRITE-3D-DOSE & -1 \\
-NORM-TYPE & -1 \\
-RANDOM-SET & -23456789 \\
! & \\
BEAM-PARAMETERS & - \\
-BEAM-WEIGHT & -100.0 \\
-DEVICE-TYPE & - \\
-DEVICE-KEY & - \\
-EVENT-NUMBER & -2000000010110 \\
-ISOCENTER-DIST & - \\
-ISOCENTER & - \\
-CHANGE-SAD & -70 \\
-GANTRY-ANGLE & - \\
-TABLE-ANGLE & - \\
-COLL-ANGLE & - \\
! & \\
END-INPUT & - \\
\hline \hline
\end{tabular}

Figure 6-5: input.vmc file 


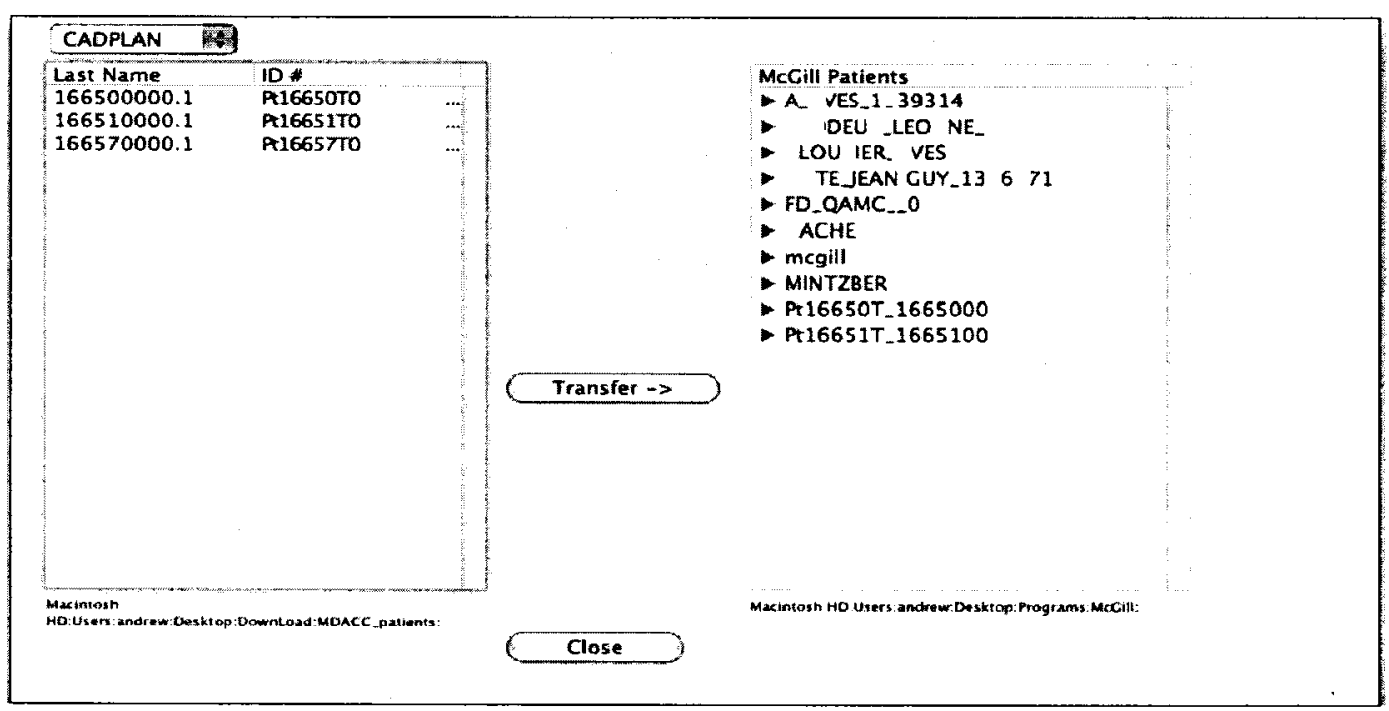

Figure 6-6: Import Window, import list-box on left and McGill RT list-box on right.

\subsubsection{External Beam Window}

The external beam window is the main program window which opens after one loads a patient. The window is comprised of a list-box, three graphic canvases and a tab menu. The list-box displays the patient plans associated to the CT set. The user can switch between plans by simply clicking on the desired plan. There is also a contextual menu for each plan. These menus hide or display the available dose distributions for a particular plan. In order to visualize a dose distribution, the user must first select it by clicking it. The user can add or delete plans by right clicking on the list-box.

The three canvases display images, contours and dose distributions. The relative size of all three can be adjusted using the horizontal and vertical slide bars. Each canvas can display three views: axial, sagittal or coronal. These views are changed by right clicking on the canvas. 
The tab menu splits the bottom portion of the beam window into four sections. These tab menus include: External Beams, Monte Carlo, Image and Dose.

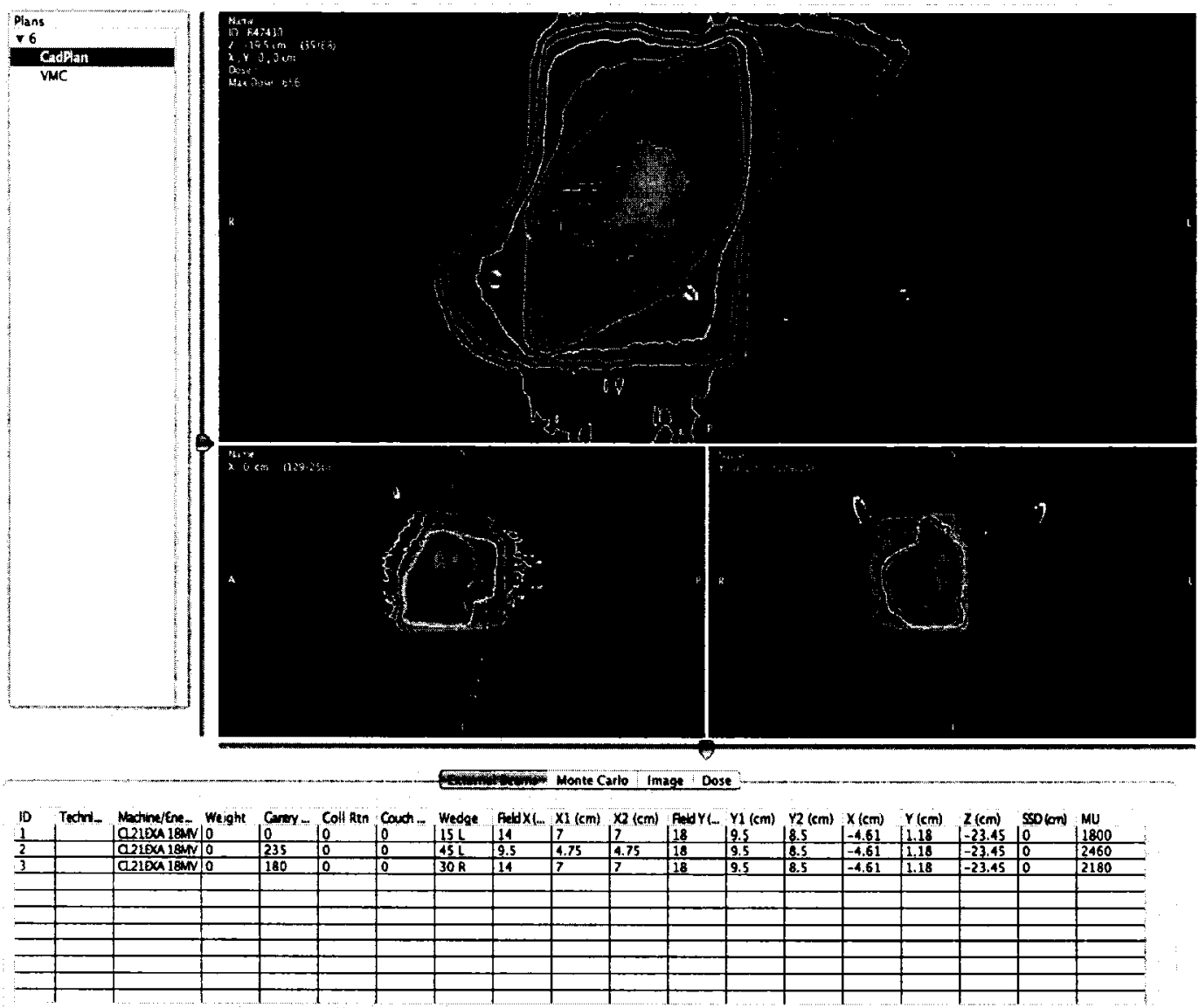

Figure 6-7: External Beam Window

\section{External Beams}

The external beam tab as shown in Figure 6-8, lists the beams associated to a plan within a list-box. The list-box also displays various beam properties for each beam. Most of these properties are editable within the list-box. There is also a beam properties window which allows access to all the beam properties. The user must 
double click on a beam to open the properties window. A right click on the list-box allows the user to add or delete a beam.

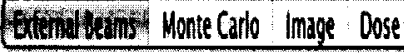

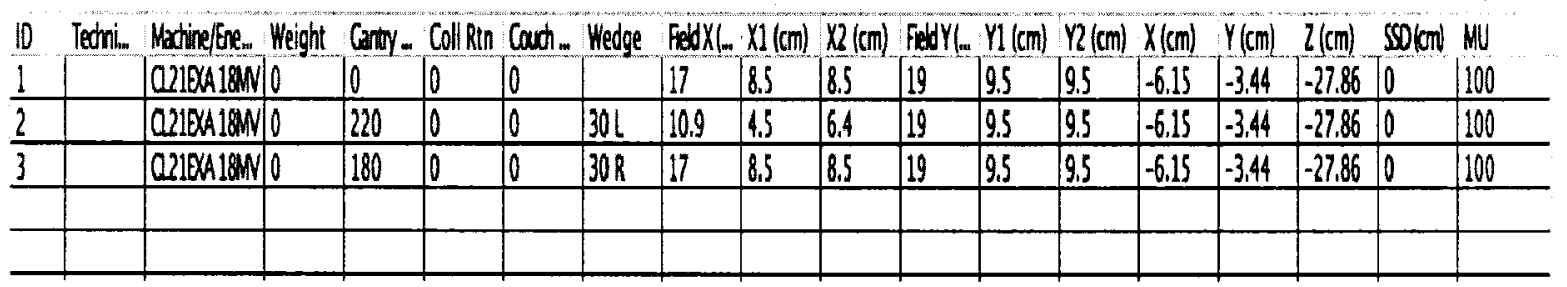

Figure 6-8: External beam tab

\section{Image}

The image tab as shown in Figure 6-9, lists various canvas display options. The window and level adjustments change the image brightness and contrast levels. There exists two slide bars for these adjustments. After an adjustment, the GUI will recalculate all images to the current window and level. Below the window and level, there is a slide bar to set the scale value. The scale value will scale the images, contours and dose distributions. Each canvas has its own scale value. To switch between these scale values, the user must right click on the scale bar and select the desired canvas.

In the middle of the image tab there exists a column of check boxes. These check boxes determine what is displayed on the canvases. The display options include, contours, colourwash dose distributions, isodose lines, image and cross-hairs. The contours and colourwash dose distributions also have a transparency setting so the user can view overlapping objects. The cross-hairs display a sagittal cross-hair and 
coronal cross-hair on the axial image and a axial cross-hair on the sagittal and coronal images. These cross-hairs allow the user to quickly scan through the patient from one image to another image. They also provide a visual reference as to the location of the image within the patient. Beside the isodose check box there is a isodose properties button. This button opens up a properties window which allows the user to define the isodose lines.

At the right hand side, there is a list-box which displays a list of the structure contours and some visualization options. These options include the structure colour, fill and show. The structure colour is changed by clicking on the colour box and selecting a new colour. The fill and show options are check boxes which determine whether or not to fill the structure and whether or not to show the structure.

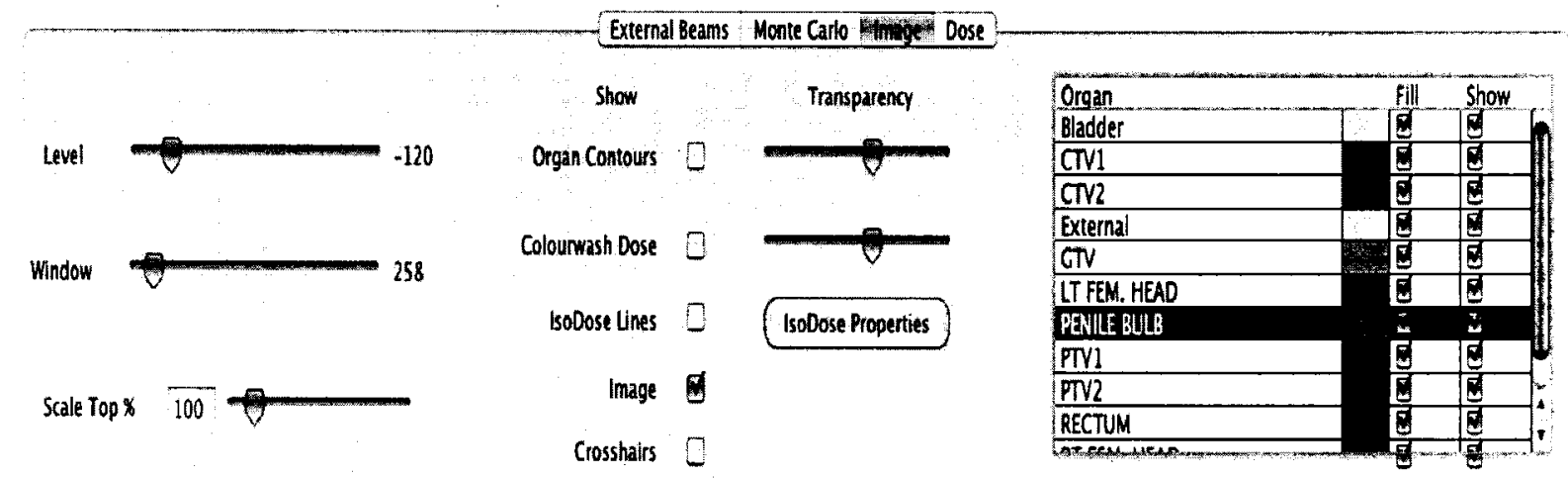

Figure 6-9: Image tab

\section{Monte Carlo}

The Monte Carlo tab handles the BEAM and VMC events. Since the Monte Carlo simulations are run on a remote cluster, there are a few button here for the shell terminal. The log in button opens up the log in properties where the user can change 
the login IP, user name and password. There is also a shell button which displays the text from the shell terminal. The third button, Kill Shell, terminates the shell. The user must use some caution with this button as it will terminate the shell regardless of the shell operation. If selected, the refresh check box will automatically check the status of the simulations for both BEAM and VMC. A second tab menu separates the BEAM and VMC buttons. The BEAM tab includes a properties button, run button and a list-box. The properties button allows access to the job split number and the number of initial particles. The list-box displays the BEAM simulation progress. The run button generates the BEAM input file, uploads the input file to the cluster and submits the job to the queue. The BEAM tab is shown in Figure 6-10.

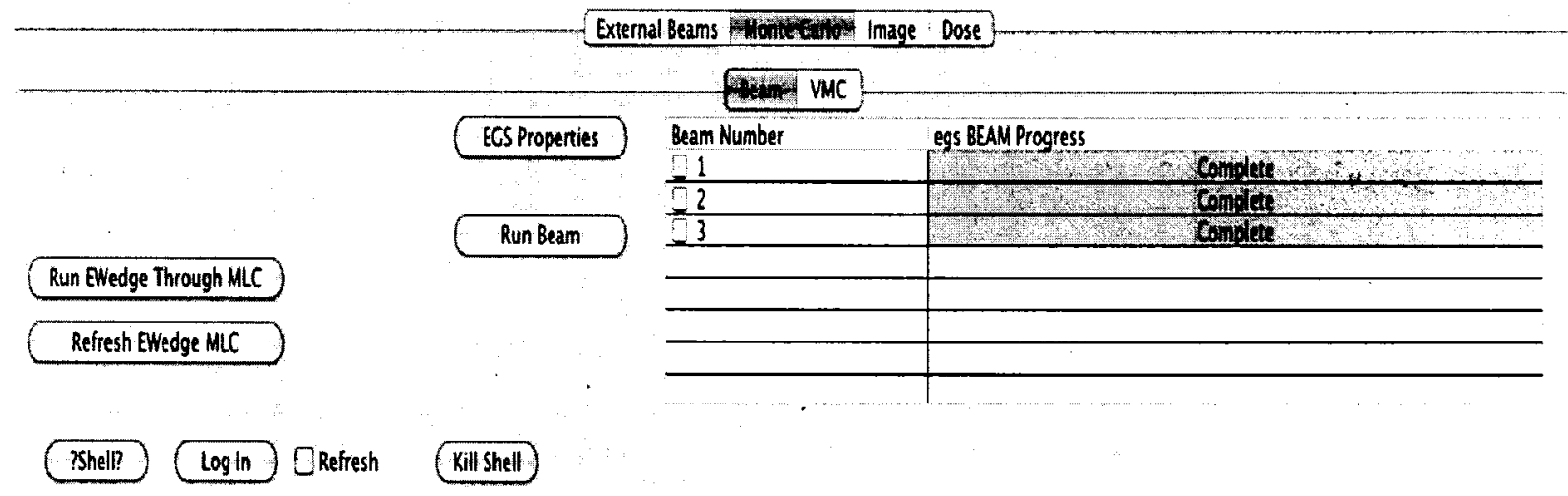

Figure 6-10: BEAM tab

The VMC tab includes the DMX, VMC options and a progress list-box. The DMX buttons allow access to the structure densities and slice selection options. The upload button generates the DMX file and uploads it to the cluster. The VMC properties include the XVMC calibration dose and the dose to water correction. 
After a simulation is complete, the GUI downloads the.$d 3 d$ file. The Add Dose button adds the.$d 3 d$ files from all beams to produce the VMC dose distribution.

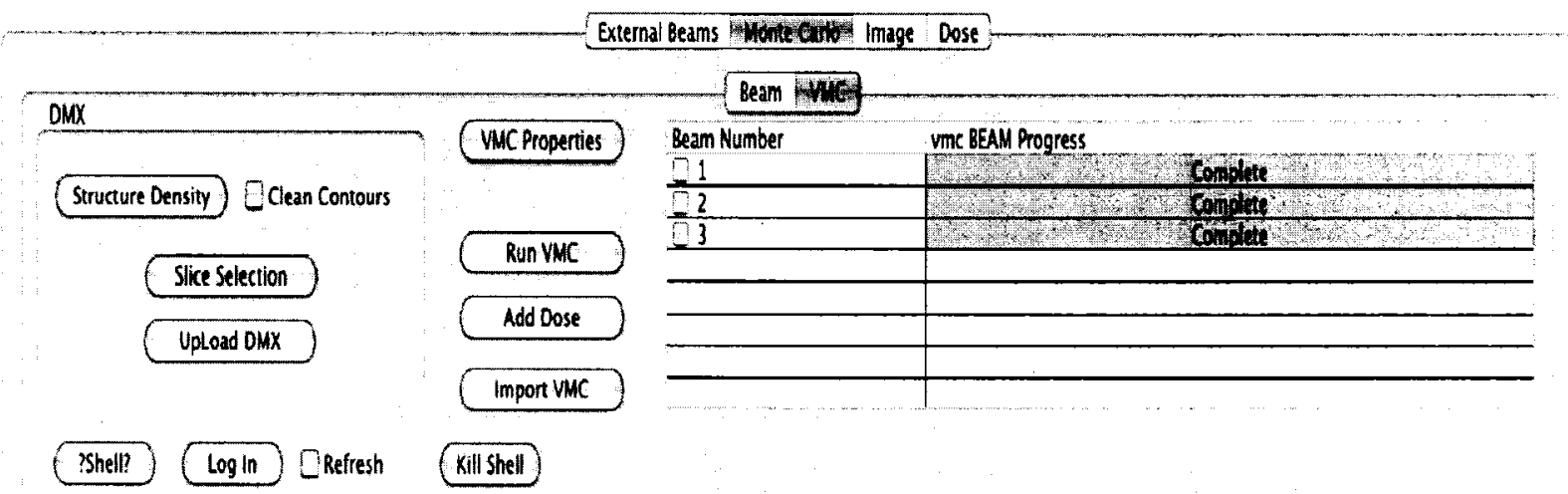

Figure 6-11: VMC tab

\section{Dose}

The dose tab as shown in Figure 6-12 holds the DVH calculator and the dose comparison tools. The DVHs are calculated and stored in memory for all structures. Once calculated, the GUI lists them in the DVH list-box. There is also an option to load CADPlan calculated DVHs. The DVH window is shown in Figure 6-13. Within the DVH window, there is the option of viewing the differential or cumulative DVH. The DVHs maybe viewed individually or all together.

The dose comparison tool allows the addition, subtraction, multiplication, and division of two dose distributions. The second dose distribution maybe be replaced with a constant. The GUI generates a new dose distribution based on the selected operation. The newly generated dose distribution is automatically saved under the appropriate plan folder. There is also the option to "paint" a dose distribution. The user generates a grid and manually paints the dose grid values one plan at a time. 


\begin{tabular}{|c|c|c|c|c|c|c|c|}
\hline Prescription & \multicolumn{2}{|l|}{$\mathrm{DVH}$} & \multicolumn{5}{|c|}{ Dose Comparison } \\
\hline & & IXCadPlan & Paint Dose & $\mathrm{CC}_{\mathrm{C}}=\overline{0}$ & copssep & 7 Copylif Oshow Crid & Save... \\
\hline & Show & & Dose Maths... & & & CadPlan & \\
\hline & Motion... & & & & & $\begin{array}{l}\text { VWC } \\
\text { VMC_Import }\end{array}$ & Process \\
\hline & Import CAD & & & & & & \\
\hline
\end{tabular}

Figure 6-12: Dose tab

This tool is currently used to quantify the DVH calculation accuracy. It's future role will be to define constraints for inverse planning techniques.

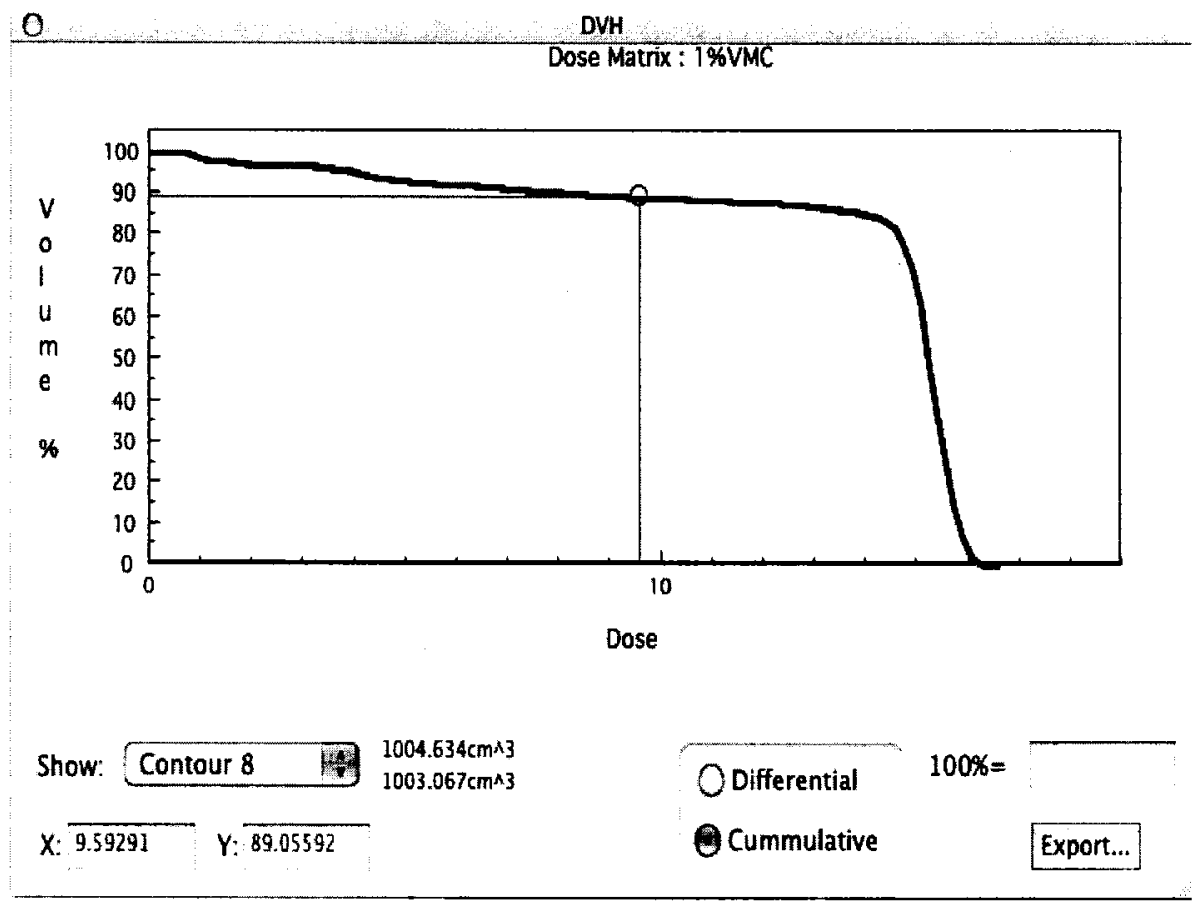

Figure 6-13: DVH window 


\subsubsection{Contouring Window}

The contouring window was only designed for structure contouring. Within this window, the user may create, edit or delete structures. This window includes one main canvas for editing contours, thumbnail image previews, a list of structures and some display options. The contours are edited individually on the axial slices. On each slice, the contour may have multiple segments. While editing a segment, the edit options include; add point, move point, delete point, scale segment, move segment, and copy segment to superior or inferior slice. The display options allow the user to change the image window and level, the scale size, the contour transparency settings and boolean check boxes to show the image or contours. The contour window is shown in Figure 6-14.

\subsubsection{MMCTP Overview}

The open patient window is the first window that appears when the user runs MMCTP. From the open patient window, the user can open a patient or switch to the transfer patient window. The transfer patient window can only transfer patient data from external formats into McGill RT and thus the user must switch to the open patient window to open a patient and proceed to the next set of windows. After the user opens a patient, the external beam window pops up. The user can access the main features of MMCTP, with the exception of contour editing, within the external beam window. Contour editing is done within its own window which the user can switch to from the external beam window. The external beam window is broken up into sections with a tab menu. Each tab allows access to various properties associated to the tab menu. A general flow chart of MMCTP is shown in Figure 6-15. 

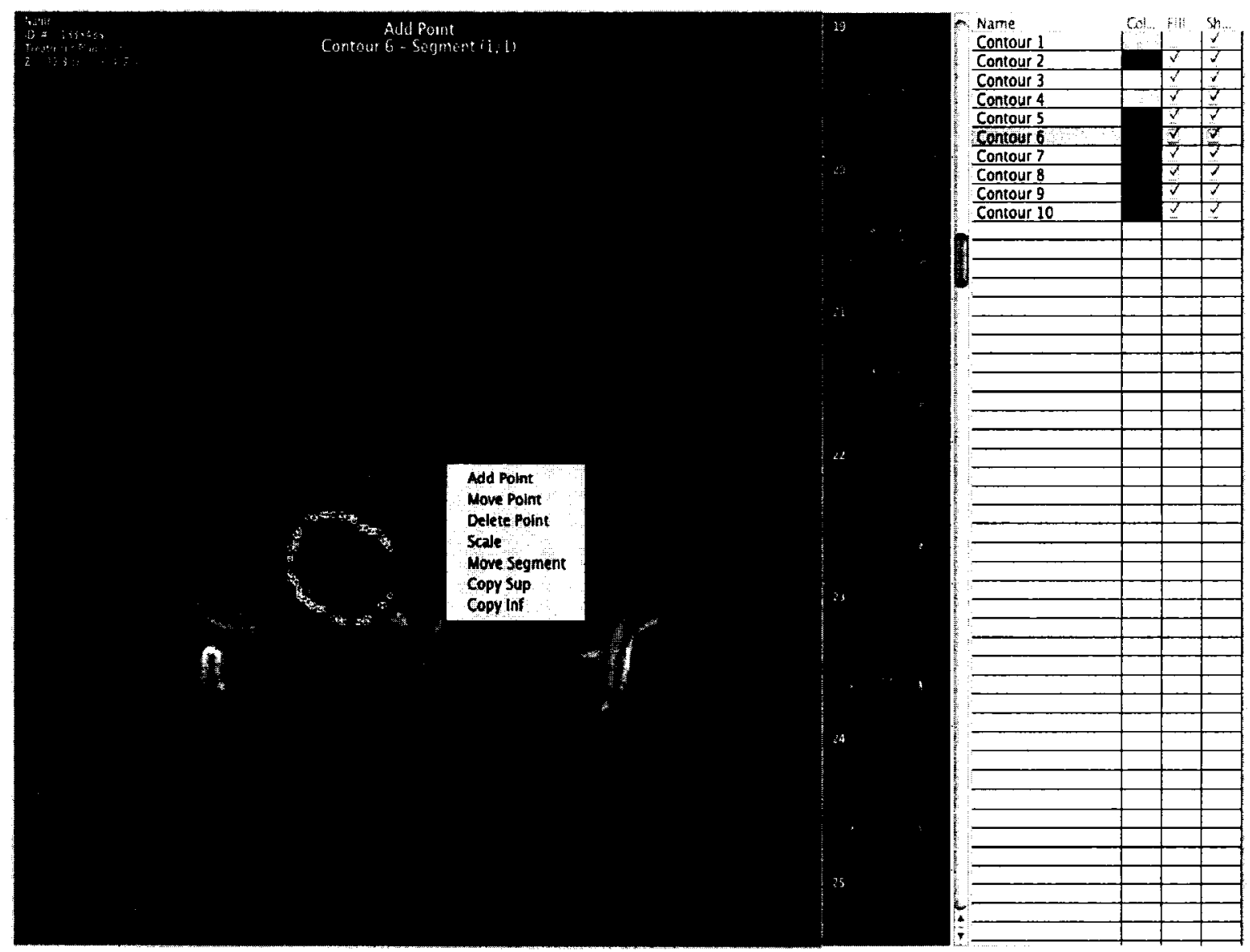

Segment 1 月

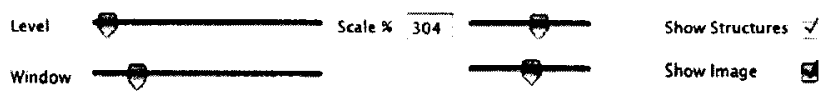

Figure 6-14: Contour window 


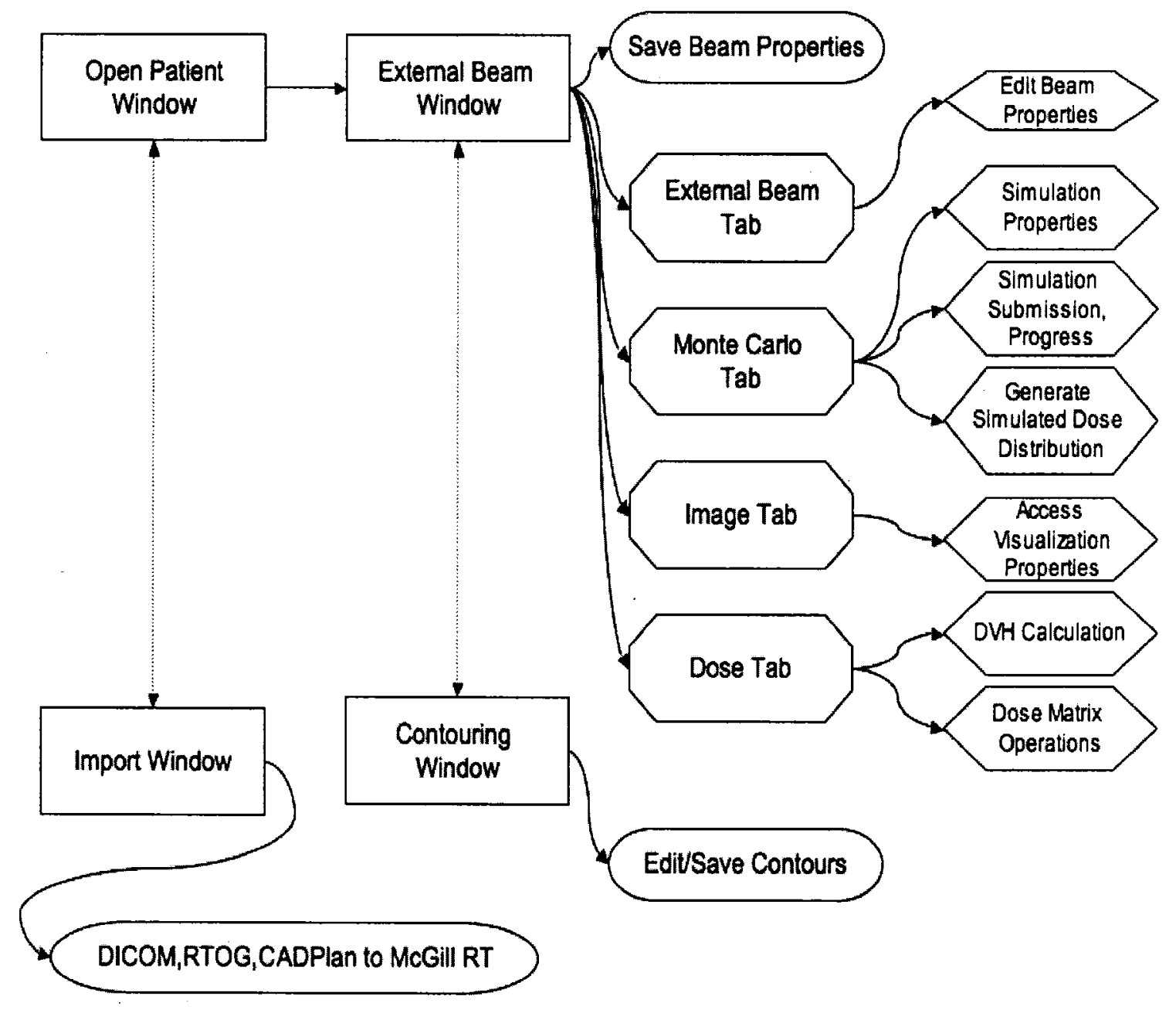

Figure 6-15: MMCTP user flow chart 


\subsection{MMCTP Testing}

MMCTP testing began once the Monte Carlo module could remotely connect to a cluster. MMCTP testing involves DVH verifications, measurement comparisons and patient dose recalculations to check the consistency of coordinate transformations, implementation of beam settings, wedge orientations, etc. By using calculations under simplified conditions (e.g., patient data replaced by homogeneous water), and comparison of the dose calculation against a conventional planning system, many consistency features were verified.

\subsubsection{DVH Verification}

As mentioned in Section 5.4.2, analyzing and quantifying the dose distribution is the most important aspect of treatment planning. It is essential for MMCTP to have an accurate DVH calculator. The DVH graphs are calculated by interpolating the dose distribution onto the $\mathrm{CT}$ resolution. The dose is then binned within the structure volumes using the structure contours and CT resolution with slice thickness as the finite volume element. Two techniques were used to assess the accuracy of the DVH calculator. The first technique compared the MMCTP DVH graphs with DVH graphs generated by commercial software packages. This comparison revealed some minor differences between the two DVH graphs. It was determined that this comparison is slightly limited by the secrecy of commercial software DVH algorithms and an exact match would not be achievable. A second approach was designed to provide an absolute method for DVH calculation and comparison. This approach uses the dose painter to paint a simple dose distribution. The distribution can be a series of lines or simple geometric objects. With simple objects, one can manually 
calculate the DVH. The idea is to compare the manual DVH with the MMCTP generated DVH.

\section{MMCTP DVH Volumes}

The first DVH test examined the accuracy of the calculated structure volumes. Within MMCTP, the structure volumes are stored as an array of coordinate points in $\mathrm{cm}$. These points are used to calculate the exact geometric polygon volume. However, this volume is not used in the DVH calculation. Instead, the volume is calculated by summing the pixels within the structure. To calculate a structure volume, each structure is painted onto a blank image with the same pixel resolution as the CT images. The volume is calculated by summing the number of painted pixels and multiplying this number by the physical dimensions of a pixel. There is often a small difference between the geometric and pixel volume. This difference is due to the partial volume effect of painting a continuous object onto a finite resolution grid.

\section{MMCTP DVH Calculations}

The DVHs are calculated by interpolating a dose value at the center of each pixel for all pixels within a structure. Where the pixel resolution represents the Dose distributions were manually created to test the accuracy of the DVH calculator. The dose distribution in Figure 6-16 was one distribution used for this test. For this distribution, the DVH calculation matched the manual calculation. Also, the dose distribution dimensions in $\mathrm{x}$ and $\mathrm{y}$ are the same dimensions as the CT image dimensions. 


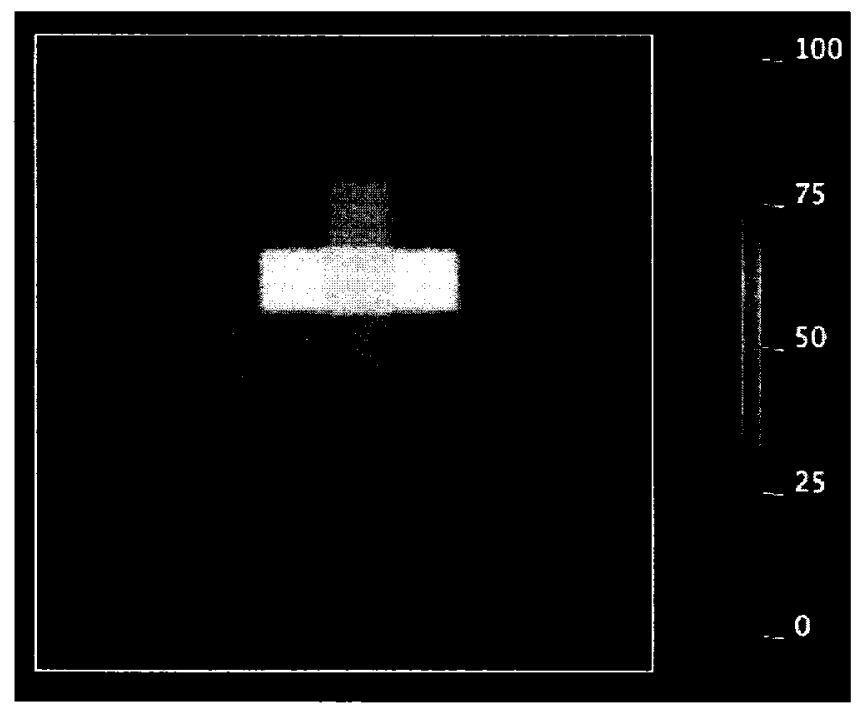

Figure 6-16: Painted dose distribution with various dose values. This distribution has the same dimensions as the CT image dimensions, thus the calculated DVH matches the manual DVH

There are some situations where the DVH calculation will not match a manual calculation. This happens when the dose distribution dimension is not a multiple of the CT image dimensions. Under this situation, the interpolation position varies within the dose distribution grid from pixel to pixel. The following two DVH graphs 6-17 demonstrate this effect. The dose distribution in Figure 6-17(a) and Figure $6-17(\mathrm{~b})$ is very similar with the only difference being a slightly higher number of dimensions in 6-17(b). This alters the DVH's appearance because the interpolation averages the dose values. This effect is only noticeable for dose distributions with a low amount of dose points and thus is not of any great concern when calculating the DVHs for patient dose distributions. 


\section{DVH Calculation Comparisons}

Dose distributions were imported from CADPlan to asses differences between DVHs calculated with CADPlan and MMCTP. The CADPlan DVH calculation algorithm uses a $1 \times 1 \mathrm{~mm}$ axial grid and interpolates the contours and dose distributions between slices. MMCTP does not use the same approach and thus, the DVHs are slightly different. Since the DVH calculations are based on structure volumes, a small study was completed to examine the calculated volume accuracy of CADPlan and MMCTP [32]. Structures were manually drawn in CADPlan and then transfered to MMCTP. The structures were basic geometric objects, triangles and boxes and the volume was manually calculated. Table 6-1 summarizes the study results. MMCTP is more precise than CADPlan on the volume calculations. Small structures tend to have the greatest error in volume. These smaller structures tend to have a

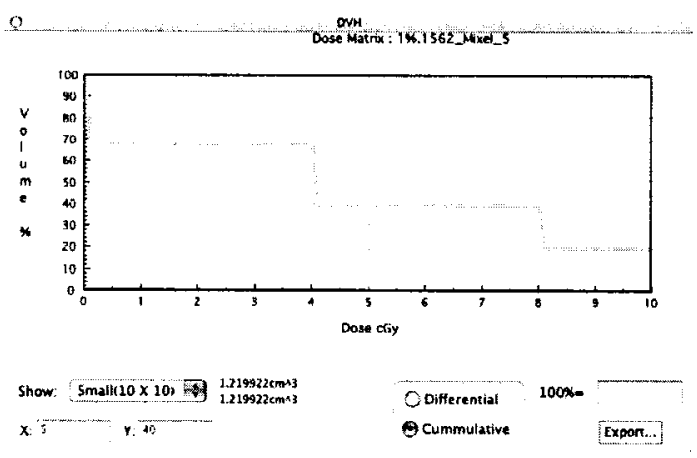

(a)

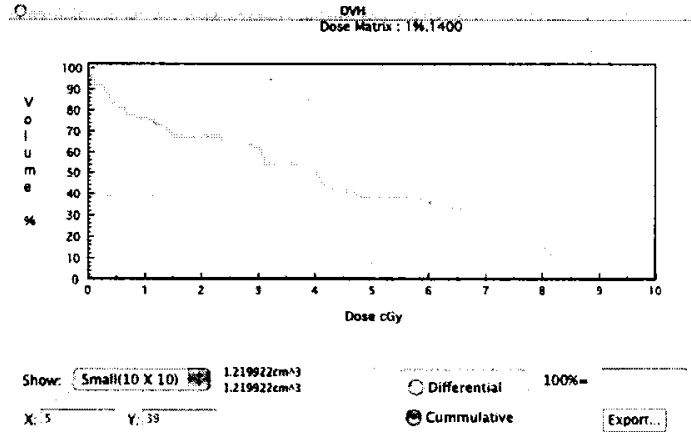

(b)

Figure 6-17: DVH comparison between dose distributions with different dimensions. (a) Dose distribution with same dimensions as CT image. (b) Dose distribution with different dimensions than CT image. The DVH in (a) matches the manual calculation while the DVH in (b) does not match because of the interpolation which averages the dose values 
greater error because there is a higher ratio of edge pixels versus internal pixels. The structure error lies in the approximation of transferring a curve onto a finite pixel resolution image. Since the DVH is normalized to the total volume, these effects are not immediately obvious. It is unknown as to why CADPlan has such a high error in calculating the structure volume. It is possible that CADPlan alters the first and last contoured slice to smooth the structure volume. This would explain why the calculated volume of basic geometric objects do not match.

Table 6-1: DVH volume calculations; CADPlan calculated volumes are not as accurate as MMCTP volumes. As the volume size decreases to small volumes, the error increases.

\begin{tabular}{ccccc}
\hline \hline Volume & \multicolumn{2}{c}{ CADPlan } & \multicolumn{2}{c}{ MMCTP } \\
$\mathrm{cm}^{3}$ & $\mathrm{~cm}^{3}$ & error (\%) & $\mathrm{cm}^{3}$ & error (\%) \\
\hline 1000 & 906.6 & 9.3 & 999.4 & 0.1 \\
700 & 612.3 & 12.5 & 699.6 & 0.1 \\
175 & 156.2 & 10.8 & 174.9 & 0.1 \\
87.5 & 78.1 & 10.8 & 87.4 & 0.1 \\
14 & 11.1 & 21.0 & 12.3 & 12.1 \\
\hline \hline
\end{tabular}

DVH curves calculated from the same dose distribution in CADPlan and MMCTP are shown in Figure 6-18. Structure volumes were compared between CADPlan and MMCTP to correlate differences in the DVH curves. The volume differences were all less than $1 \%$ with the exception of the heart. The heart has a volume difference of over $4 \%$. This is certainly a contributing factor to the heart DVH difference. Despite an excellent volume agreement, the CADPlan lung curve is consistently lower than the MMCTP curve. These differences are likely due to the dose interpolation between slices. In general, when comparing DVH's from two different engines one 
should use the same DVH calculator and make sure the dose distributions are resolved on the same calculation grid. This will ensure differences observed within the DVH curves are attributed to differences in the dose distribution and not the DVH calculation method.

\subsubsection{Measurement Comparisons}

A few simple experiments were designed to compare the measured dose with the simulated VMC dose. The experimental equipment included a Solid Water ${ }^{\mathrm{TM}}$ phantom, CT scanner, ion chamber (NE 2571), film (EDR2, KODAK) and a Varian 21EX linac (Varian, Inc. Palo Alto, CA). The Solid Water ${ }^{\mathrm{TM}}$ phantom was first CT scanned to generate DICOM ${ }^{*}$ images for MMCTP. The phantom consists of three slabs of Solid Water $^{\mathrm{TM}}$ producing a block of Solid Water ${ }^{\mathrm{TM}}$ with dimensions $\left(20,20,20 \mathrm{~cm}^{3}\right)$. The ion chamber slides within the phantom and its sensitive volume is located at the center of the phantom. After importing the DICOM images, plans were generated, calculated and delivered to the water phantom with the linac. The plans tested various beam properties for example, beam energy, gantry angle, jaws, wedges and MLC fields. The calculated dose distributions were then compared to the film measurements and ion chamber readings.

\section{Film Measurements}

Film may be used as an absolute or relative dosimeter. The following comparisons examine the relative dose distribution between the measured film and calculated distribution. For each measurement, a film was positioned between two of the solid water slabs. The film is perpendicular to the beam axis for a gantry angle of $0^{\circ}$. This means the measurements were in the coronal plane. After the measurements, the 


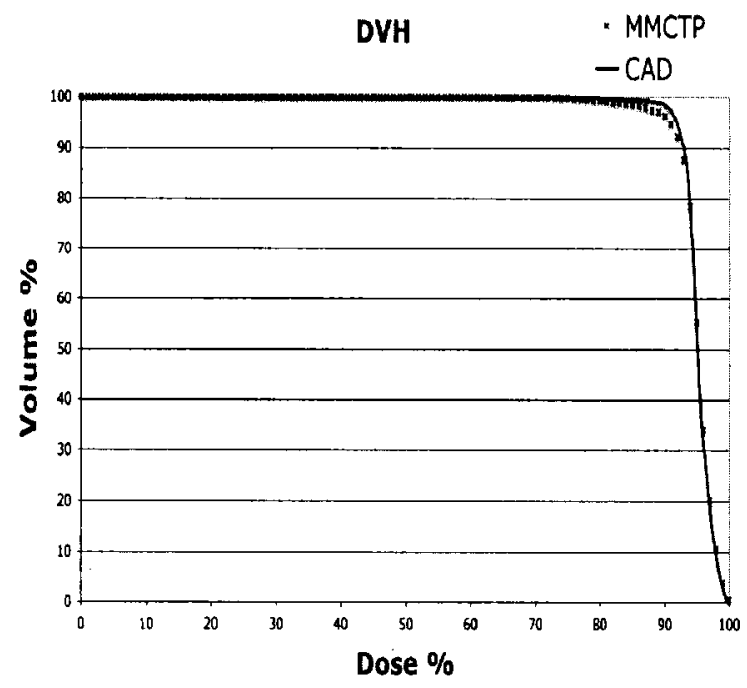

(a)

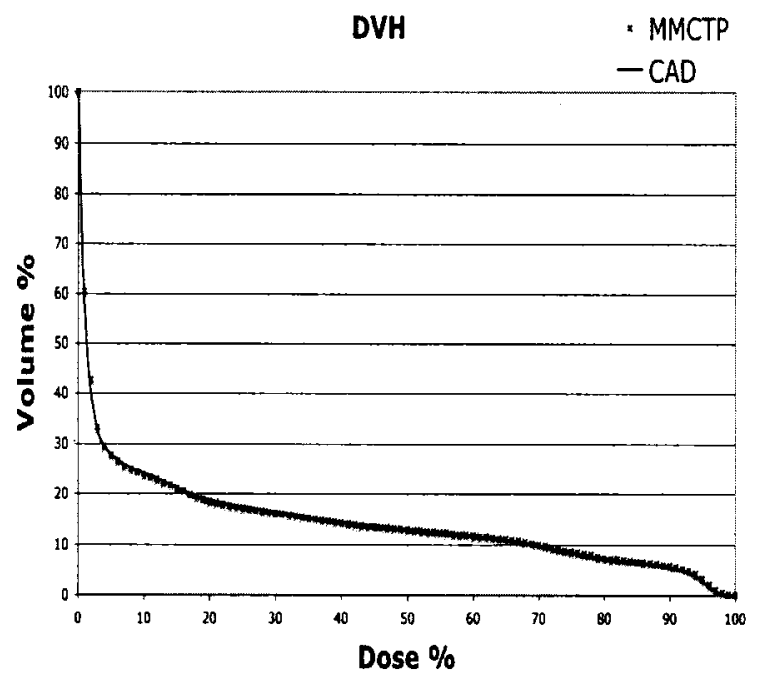

(c)

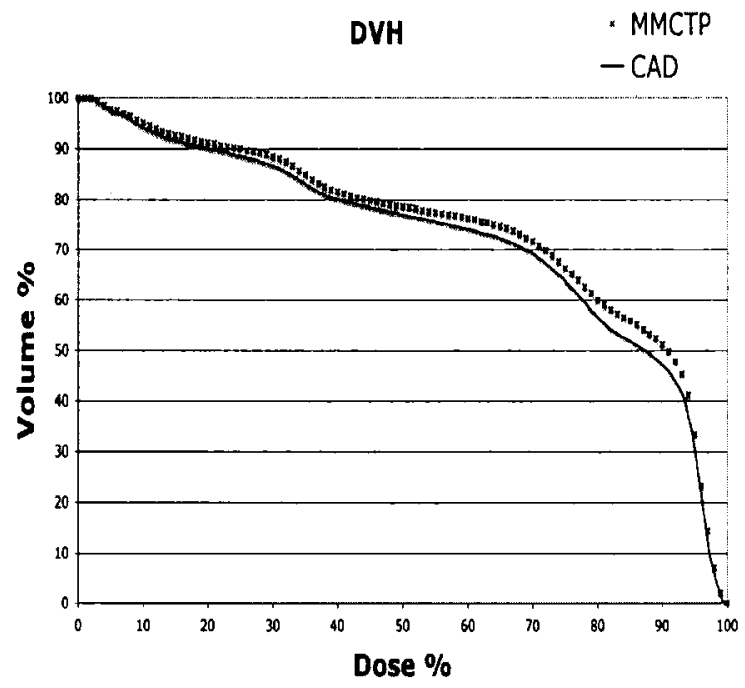

(b)

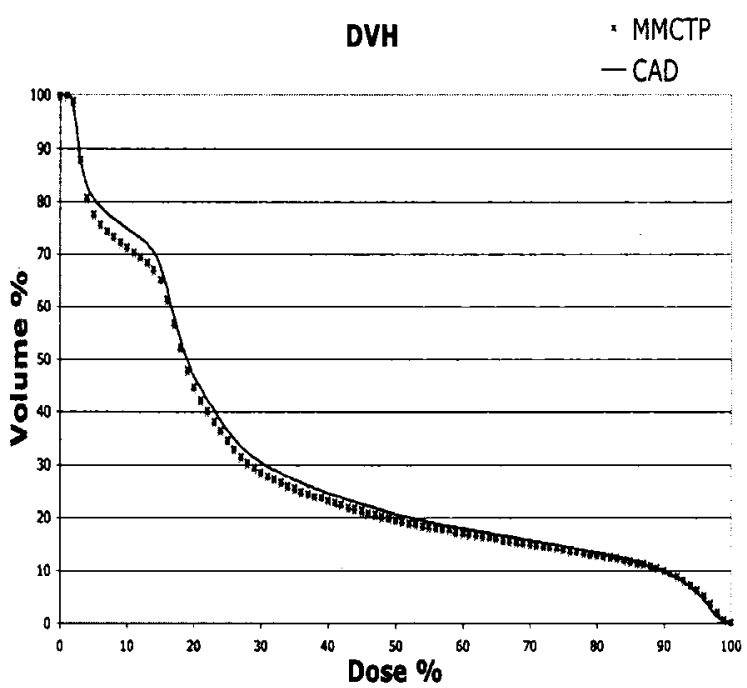

(d)

Figure 6-18: DVHs calculated on CADPlan and MMCTP (a) CTV (b) Lung (c) External (d) Heart 
film was processed, scanned and saved as a tiff image. The images and VMC dose distribution were analyzed in matlab using the gamma $(\gamma)$ technique [13]. The $(\gamma)$ technique was developed to qualitatively compare dose distributions, either measured or calculated [12]. A $\gamma$-index distribution can be generated and displayed, providing a quantitative assessment of the quality of the calculation both in regions that pass and fail the acceptance criteria [13]. Equation 6.2 is used to define the gamma index at each point in the evaluation plane $r_{c}-r_{m}$ for the measurement point $r_{m}$,

$$
\gamma\left(r_{m}\right)=\min \left\{\Gamma\left(r_{m}, r_{c}\right)\right\} \forall\left\{r_{c}\right\}
$$

where

$$
\begin{gathered}
\Gamma\left(r_{m}, r_{c}\right)=\sqrt{r^{2}\left(r_{m}, r_{c}\right) \Delta d_{M}^{-2}+\delta^{2}\left(r_{m}, r_{c}\right) \Delta D_{M}^{-2}} \\
r\left(r_{m}, r_{c}\right)=\left|r_{c}-r_{m}\right|
\end{gathered}
$$

and

$$
\delta\left(r_{m}, r_{c}\right)=D_{c}\left(r_{c}\right)-D_{m}\left(r_{m}\right)
$$

The acceptance criteria is chosen with regards to the resolution of the distributions. In general, the minimum distance criteria should be three times the resolution size and the dose difference criteria should be $3 \%$. The pass-fail criteria therefore becomes: 


$$
\begin{gathered}
\gamma\left(r_{m}\right) \leq 1 \text { calculation passes, } \\
\gamma\left(r_{m}\right)>1 \text { calculation fails. }
\end{gathered}
$$

Two $\gamma$ comparisons between measurements and simulations are shown in Figure 6-19 and Figure 6-20. Both figures show good agreement within the beam field. Outside the field, the $\gamma$ index is above 1 and the test fails. This is expected because these are low dose regions with high uncertainty. In Figure 6-19, the beam properties are; $6 \mathrm{MV}$ photon beam, $10 \times 10 \mathrm{~cm}^{2}$ jaw opening, $15^{\circ}$ wedge and a gantry angle of $45^{\circ}$. In this figure, the $\gamma$ index is below 1 for pixels within the beam field. In figure 6-20, the beam properties are; $6 \mathrm{MV}$ photon beam, MLC pattern, and a $15^{\circ}$ wedge. The $\gamma$ index is below 1 around the MLC pattern, but there is a region within the beam field where the $\gamma$ index rises above 1. Overall, there is good agreement between the measured and calculated distributions.

\section{Ion Chamber Measurements}

The ion chamber measurements were used to verify the absolute dose generated from Monte Carlo simulations. The dose was calculated using a calibrated ion chamber. Equation 6.5 relates the chamber readings to dose to water, where $\bar{R}, P, T$, and $\left(N_{D, W}\right)_{S W}$ represent the average chamber reading, pressure reading, temperature reading and the air chamber calibration factor. An additional correction was applied to the chamber reading to account for linac output changes. The linac output per MU should remain constant but it is normal to observe small changes. In order to 


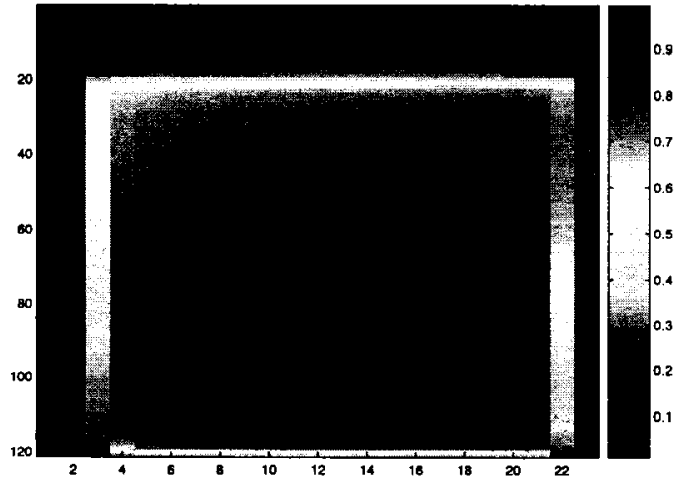

(a)

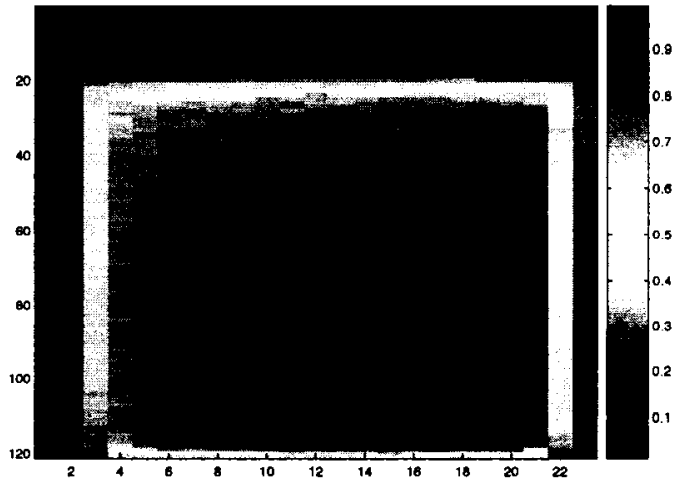

(b)

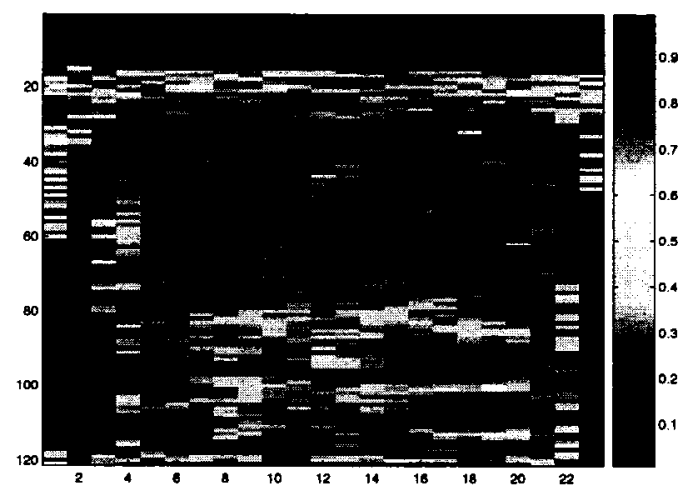

(c)

Figure 6-19: Simulated and measured dose distributions for $6 \mathrm{MV}$ photon beam, $10 \times 10 \mathrm{~cm}^{2}$ field size, $15^{\circ}$ wedge. (a) measured film distribution. (b) simulated distribution. (c) gamma map comparison between film and simulation, $\gamma$ values above 1 represent a disagreement between the film and simulation within the tolerance limits, $\gamma$ values above 1 are coloured red. 


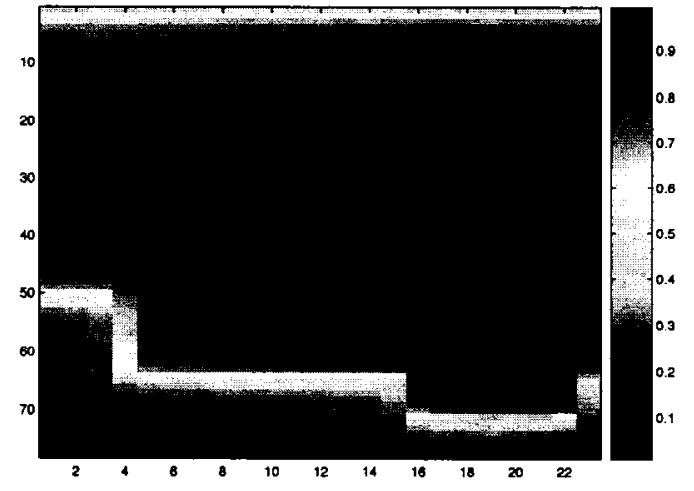

(a)

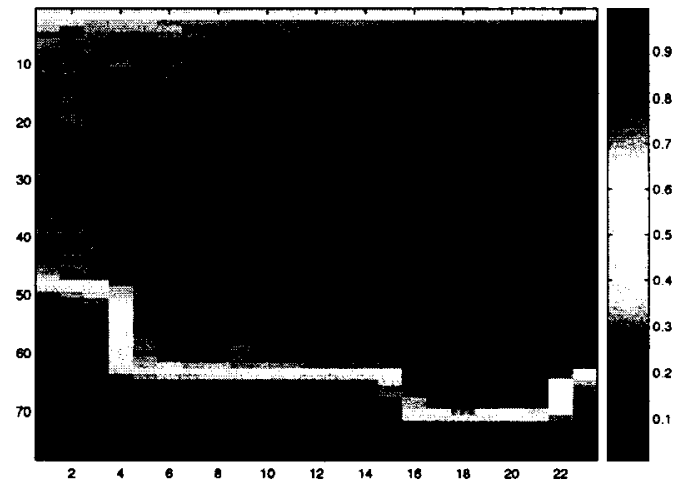

(b)

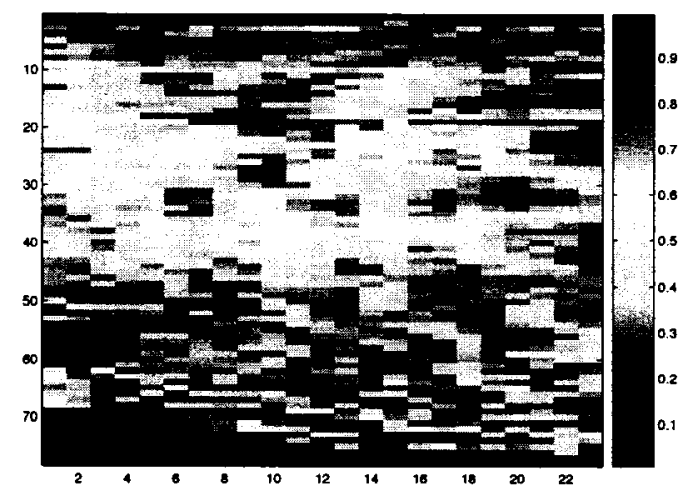

(c)

Figure 6-20: Simulated and measured dose distributions for $6 \mathrm{MV}$ photon beam, with jaws at $X_{1}=10.2 \mathrm{~cm}, X_{2}=4.3 \mathrm{~cm}, Y$ field $20 \mathrm{~cm}, 15^{\circ}$ wedge and MLC pattern. (a) measured film distribution (b) simulated distribution (c) gamma map comparison between film and simulation, $\gamma$ values above 1 represent a disagreement between the film and simulation within the tolerance limits, $\gamma$ values above 1 are coloured red. 
compare a measured dose value with a simulated dose value, one must examine the linac output and correct for output changes. These changes are detectable by performing the TG-51 protocol. The protocol was written by Task Group 51 (TG-51) of the Radiation Therapy Committee of the American Association of Physicists in Medicine (AAPM) for clinical reference dosimetry of external beam radiation therapy using photon beams with energies between ${ }^{60} \mathrm{Co}$ and $50 \mathrm{MV}$ and for electron beams with nominal energies between 4 and $50 \mathrm{MeV}$.

$$
D_{w}(c G y)=\bar{R} \times\left(\frac{760}{P} \times \frac{273+T}{295}\right) \times\left(N_{D, W}\right)_{S W}
$$

To obtain the simulated chamber dose, the chamber is first contoured. The chamber was contoured within MMCTP using the contour editor and CT images. The phantom is also contoured to assign density values for the DMX generation. For these measurement comparisons, the phantom density was assigned a value of 1 $\mathrm{gcm}^{-3}$. The dose to the chamber is calculated by integrating the chamber DVH. For each simulation, there is also an associated statistical uncertainty.

The measurements were taken under standard conditions with $10 \times 10 \mathrm{~cm}^{2}$ fields and the phantom positioned using $S A D$ setup. The results are summarized in Table 6-2. In general, the agreement between measured and calculated dose is acceptable for clinical applications but the results were expected to be closer. It should be noted that there is an assumed approximation when using the $\left(N_{D, W}\right)_{S W}$ value under $S A D$ setup. The $\left(N_{D, W}\right)_{S W}$ value is used to determine the dose to water under specific conditions such as $S S D$ of $100 \mathrm{~cm}$, field size $10 \times 10 \mathrm{~cm}^{2}$ and at a specific depth in Solid Water ${ }^{\mathrm{TM}}$. The specific depth in Solid Water ${ }^{\mathrm{TM}}$ corresponds to 
a depth of $10 \mathrm{~cm}$ in water. Thus, to use a Solid Water ${ }^{\mathrm{TM}}$ phantom and $\left(N_{D, W}\right)_{S W}$ to determine the dose to water, one also has to correct for the depth in Solid Water ${ }^{\mathrm{TM}}$. A second experiment was designed using a water tank and an Exradin A12 (Standard Imaging Inc. Middleton, WI) air chamber to accurately determine the dose to water. The results of this experiment is show in Table 6-3. The water tank and air chamber were positioned at the same $S S D$ and depth as in the Solid Water ${ }^{\mathrm{TM}}$ setup in order to match the simulated dose to water value with these new measurments. The agreement between the water tank measurements and calculated dose is now within 1 $\%$. There are a few uncertainties that influence the water tank dose measurement and the calculated dose value. For the water tank dose measurement, there is uncertainty in the set-up and in the chamber calibration factor. While for the calculated dose value, there is uncertainty in the contoured chamber. With a clinical application in mind, a disagreement of less than $1 \%$ is considered an excellent match however, these uncertainties could be examined to further reduce the dose discrepancy.

Table 6-2: Dose to water in $S A D$ setup at depth of $8.44 \mathrm{~cm}$ from measurements in Solid Water ${ }^{\mathrm{TM}}$ phantom and simulations

\begin{tabular}{llllllll}
\hline & \multicolumn{2}{c}{ Beam Properties } & \multicolumn{3}{c}{ Measured } & \multicolumn{2}{c}{ Simulated } \\
\hline Energy (MV) & Field $\left(\mathrm{cm}^{2}\right)$ & $S A D(\mathrm{~cm})$ & MU & Dose (cGy) & $\sigma \%$ & Dose (cGy) & $\sigma \%$ \\
\hline 6 & $10 \times 10$ & 100 & 100 & 84.4 & 0.1 & 85.6 & 0.5 \\
18 & $10 \times 10$ & 100 & 100 & 99.89 & 0.1 & 101.7 & 0.5 \\
\hline \hline
\end{tabular}

\subsubsection{Patient Recalculations}

Various lung patients were recalculated using MMCTP as an extensive test to identify problems throughout MMCTP job control and submission mechanics. These 
Table 6-3: Dose to water in $S A D$ setup at depth of $8.44 \mathrm{~cm}$ from measurements in water tank and simulations

\begin{tabular}{llllllll}
\hline \hline & \multicolumn{2}{c}{ Beam Properties } & \multicolumn{2}{c}{ Measured } & \multicolumn{2}{c}{ Simulated } \\
\hline Energy (MV) & Field $\left(\mathrm{cm}^{2}\right)$ & $S A D(\mathrm{~cm})$ & MU & Dose (cGy) & $\sigma \%$ & Dose (cGy) & $\sigma \%$ \\
\hline 6 & $10 \times 10$ & 100 & 100 & 84.9 & 0.1 & 85.6 & 0.5 \\
18 & $10 \times 10$ & 100 & 100 & 101.9 & 0.1 & 101.7 & 0.5 \\
\hline \hline
\end{tabular}

patients were originally planned using CADPlan. Thus, the dose distribution was calculated using a pencil beam algorithm and assuming the patient body is homogeneous water. Patient plans were imported into MMCTP and the dose was recalculated with Monte Carlo. Throughout these tests, the MMCTP-generated Monte Carlo input files were compared with manually created Monte Carlo input files. Differences between these files indicate errors within the Monte Carlo module. It should be noted that the VMC patient specific density matrix was assigned a value of $1 \mathrm{gcm}^{-3}$. This should ensure good agreement between the CADPlan calculated distribution and the Monte Carlo distribution since CADPlan also assigns the geometry to water. Once the Monte Carlo dose distribution was calculated, a visual and DVH comparison was preformed. The visual comparison examined the isodose distribution per axial slice. Figure 6-21 shows an isodose comparison between CADPlan and Monte Carlo. The CAPlan distribution is limited to the external contour of the patient, while the Monte Carlo distribution extends into the volume of air surrounding the patient. For each distribution, the isodose lines are normalized to the maximum dose. Overall, the isodose agreement is good. 


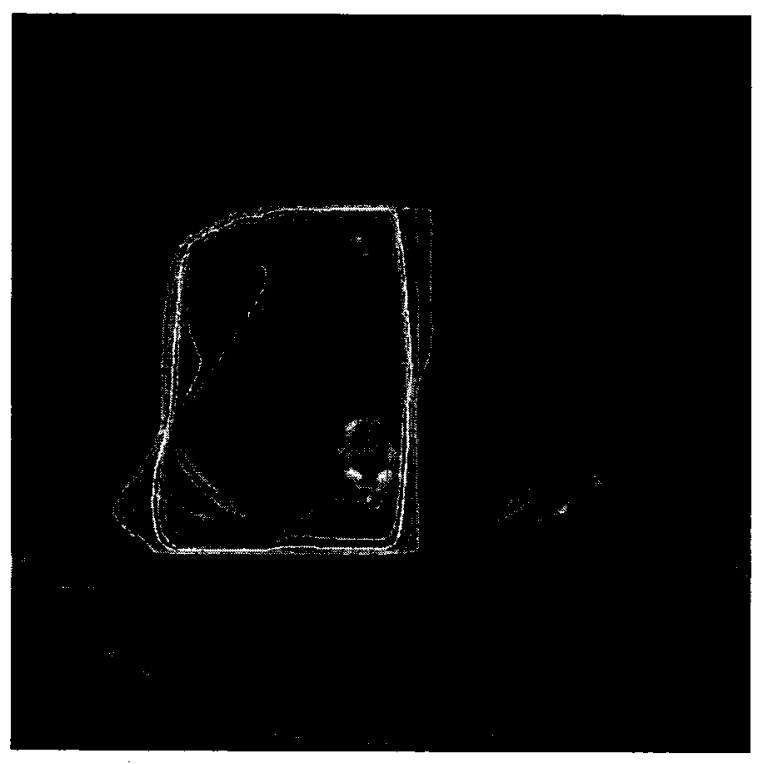

(a)

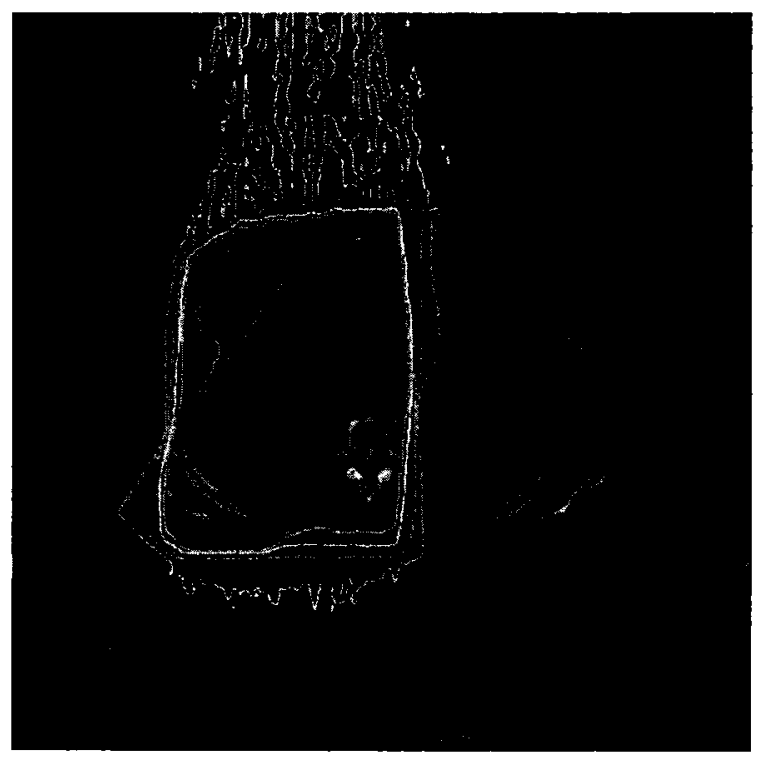

(b)

Figure 6-21: Comparison between isodose distribution. (a) Isodose distribution from CADPlan. (b) Isodose distribution from Monte Carlo.

\section{Patient Recalculations, DVH comparison}

The following Figure 6-22 displays the VMC and CADPlan DVH comparison for two lung patients. Both patients were treated with three beams at various gantry angles using wedged and MLC fields. The DVHs were calculated for three structures (CTV, Lung and Heart). The CADPlan and Monte Carlo DVHs for both patients were calculated within MMCTP in order to avoid DVH calculation differences which compromise the comparison. The CTV matches well for both patients. In both patients, the VMC heart DVH is noticeably higher than the CADPlan DVH. This can be explained by the increased penumbra in Monte Carlo fields compaired to CADPlan penumbra. In the presence of MLC shaped fields, the CADPlan pencil 
beam algorithm is used to calculate dose distributions. Diode measurements results comparing pencil beam algorithm and Monte Carlo calculations for a MLC field show that the pencil beam algorithm does not accurately predict the dose profile in the $50 \%$ to $90 \%$ region [18].

For both patients, the heart is tangential to the beam. Tangential structures receive a higher dose in Monte Carlo fields because of the increased penumbra. To demonstrate this effect, a profile comparison between VMC and CADPlan is shown in Figure 6-23. The profile was taken through the middle of the heart and reveals a higher VMC penumbra compared to the CADPlan penumbra. The lung DVH is in good agreement for patient 1 but patient 2 has a higher VMC DVH. The explanation for this increase is likely the penumbra effect. The lung is a large organ which expands past the CTV. Thus, a large percentage of the lung falls into the penumbra region.

The patient recalculations demonstrate the current abilities of MMCTP. The recalculations were an extensive test that tested the: CADPlan importer, external beam planning, Monte Carlo control and the DVH calculation. The overall agreement between the isodose distributions and the DVH comparison indicates proper MMCTP functionality. DVH differences exist between CADPlan and Monte Carlo when the treatments fields are tangental to the organ. This is due to wider beam profiles as calculated by Monte Carlo. 


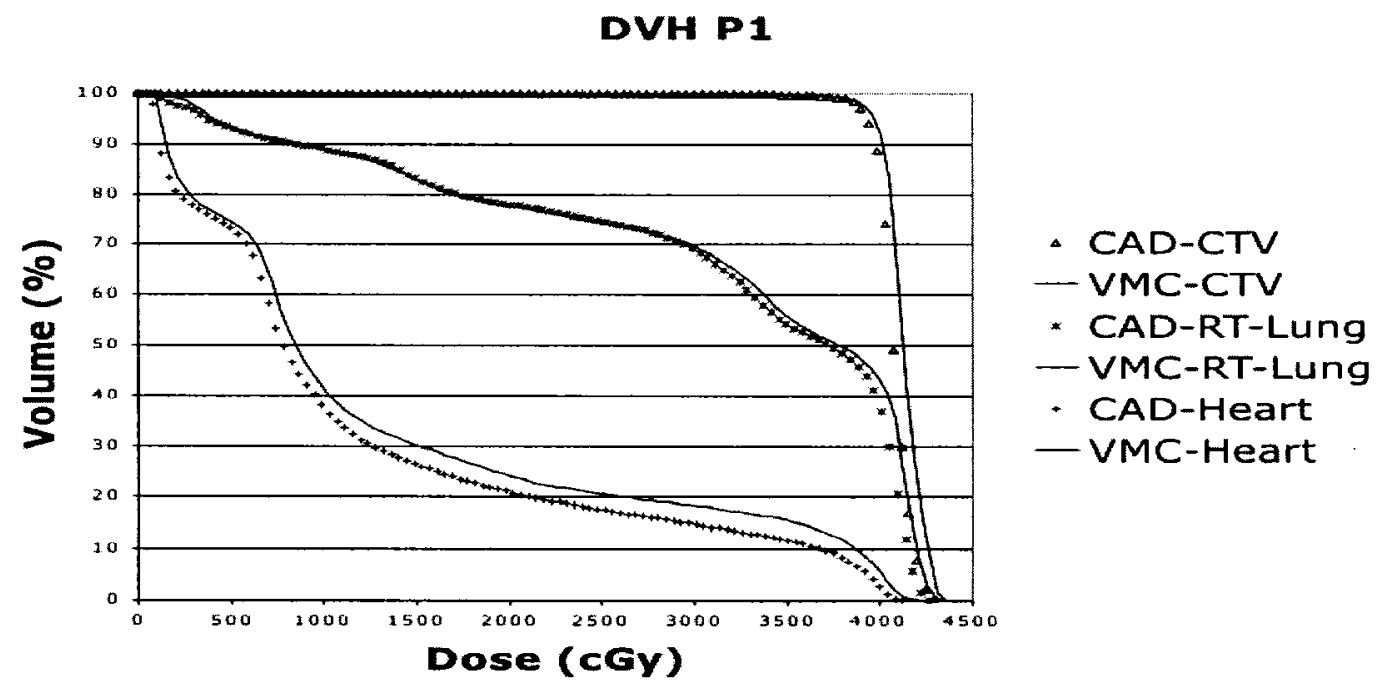

(a)

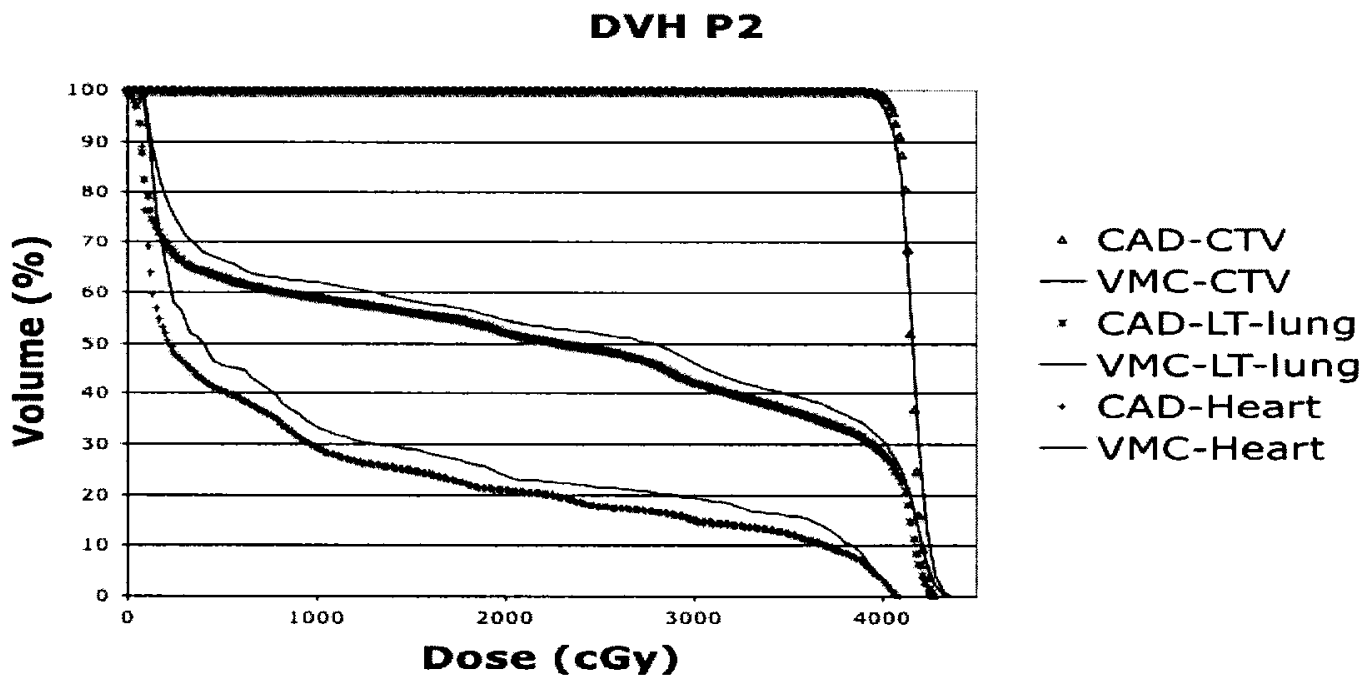

(b)

Figure 6-22: DVH comparison for patient recalculations between CADPlan and MMCTP (VMC). (a) Patient 1 VMC CTV DVH is slightly higher than CADPlan but within $1 \%$. (b) Patient 2 VMC CTV DVH is in excellent agreement with CADPlan. 


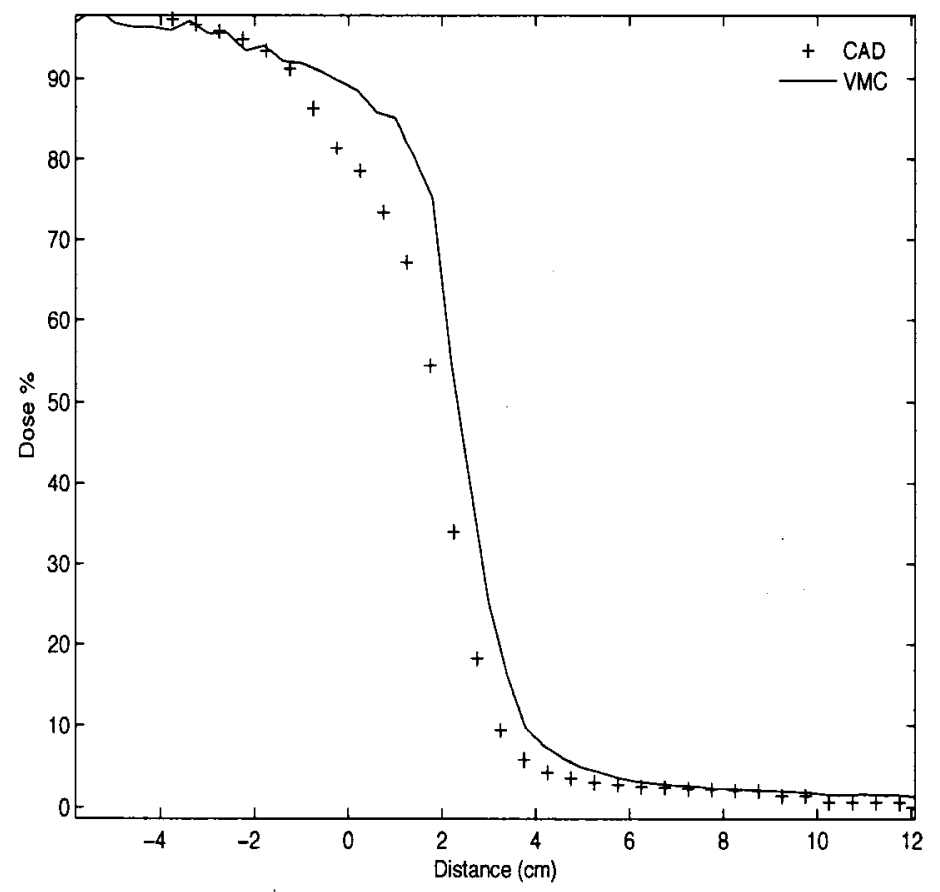

Figure 6-23: Profile comparison for CADPlan and VMC. VMC has a higher penumbra, due to CADPlan's inability to accurately model MLC fields. 


\section{CHAPTER 7 \\ Conclusion}

Monte Carlo radiotherapy treatment planning (MCTP) has only now made a slow entry in the clinical environment taking considerably longer than envisioned. The main objective of this thesis was to build a flexible computational radiotherapy research environment which allows for MCTP, outcome analysis and other future research implementations. A research environment of this type opens the possibility of large-scale retrospective and prospective Monte Carlo studies.

In this thesis, MMCTP was built as a research platform for the development of patient specific Monte Carlo treatment planning for external beam radiation therapy. The MMCTP design consists of a graphical user interface (GUI) which remotely connects to a computer cluster for Monte Carlo simulations. MMCTP uses an internal storage format that is flexible in that it allows for future implementation of multi-instance and multi-modality images. The visualization options, dose matrix operations and DVH tools offer extensive possibilities for plan analysis and comparisons. Plans are imported within MMCTP from commercial treatment planning systems through well-documented storage protocols such as DICOM_RT and RTOG. MMCTP features a MCTP architecture that uncouples the private patient data from remote calculation engines. This philosophy allows MMCTP to connect to large university-based computer grids so there is no need for expensive computer 
hardware. Potentially, MMCTP can offer clinics access to MCTP by providing nonspecialist people (dosimetrist) a simple interface for MCTP.

\subsection{Summary of work performed}

The first task of this thesis was to design and build a computational radiotherapy research platform GUI using REALbasic. The design includes a method for Monte Carlo calculations. As the GUI became operational, various validation tests were performed to ensure proper functionality.

\subsubsection{MMCTP GUI}

The MMCTP GUI was designed with REALbasic and has its own unique data storage format. The storage format, called McGill RT, was developed to organize radiotherapy data in specific files and folders. This format was based on RTOG but with efficiency improvements for reading and writing data. The RTOG format was used to design RTOG objects within the GUI. These objects are populated in memory as patient data is read into MMCTP. MMCTP can import RTOG, DICOM and CADPlan patients. Once these patients are imported, they are saved into McGill RT format. Opening a patient within MMCTP brings the user to the external beam planning window. The external beam planning window includes sections for beam editing, Monte Carlo options, dose and contour visualization options, dose matrix operations and DVH calculations. The dose distributions and contours have transparency settings and can be viewed in axial, sagittal and coronal planes. The DVHs are stored in memory and there is a separate DVH window for each dose distribution. 
MMCTP can recalculate patient dose distributions with Monte Carlo simulations as the dose engine. Monte Carlo simulations are calculated in a two-step process using BEAM and VMC. The Monte Carlo properties for BEAM simulations include the number of initial particles and the job split number. The VMC simulation properties define the density matrix generation. This matrix is either generated from converting the CT Hounsfield numbers to density values and or using the contour structures to assign density values to the individual structures. Aside from the density matrix there is a VMC dose per MU calibration value for each virtual linac energy. The dose per MU calibration value is used to normalize the dose distribution from dose per particle to dose per MU. The Monte Carlo simulations are calculated on a remote cluster and files are transferred between the GUI and cluster through sftp connections. Upon completion of VMC, MMCTP downloads the individual dose matrix files to the workstation. These files are then locally summed up to produce one dose distribution file per plan.

There is a separate contouring window within MMCTP which allows the user to generate new contours and edit existing contours. The contours are displayed and edited in the axial plane. There are various display options within this window to assist the editing process. The editing options are basic but include the necessary tools to contour structures on multiple slices.

\subsubsection{Validation}

The validation tested some limited cluster communications, Monte Carlo results, DVH calculations and patient recalculations. Communications between MMCTP and the cluster utilized the REALbasic shell. Files were transfered to the cluster 
and various commands were executed through the shell. Once the communications were determined to be working, Monte Carlo testing involved comparisons between simulations and measurements (film, ion chamber). The film measurement provides a relative two dimensional dose distribution while the ion chamber provides an absolute dose measurement. The gamma map comparison shows good agreement between the film and Monte Carlo distribution. The absolute dose measurements were compared to the average dose within the contoured ion chamber and the results were within acceptable limits. Patient recalculations were performed as a validation step to identify potential problems throughout the Monte Carlo process such as wedge orientations, coordinate systems, couch gantry and collimator angles. A patient recalculation with the patient volume set to water should produce an identical dose distribution as the conventional uncorrected plan. Two patients were recalculated with the patient volume set to water to test the Monte Carlo process. The DVHs from the Monte Carlo and conventional plan matched well for the target contours within the beam but there are differences in the DVHs for contours within the penumbra regions. These differences arise because of the inaccuracies in beam modeling for conventional dose calculation algorithms. The overall agreement with the measurements and patient DVHs support the validation process and indicates proper functionality.

\subsection{Future work}

There are an endless amount of features to incorporate into MMCTP. Electron beam planning is one example which has been partially implemented. Most often, electron beam planning utilizes charts and tables to approximate the dose 
distribution. Monte Carlo electron planning offers accurate patient specific dose distributions. Another useful feature will be IMRT Monte Carlo optimization. A third feature could be to offer a second dose calculation model as an alternative to VMC. A fourth feature is to incorporate energy modulated electron therapy. Energy modulated electron therapy developed at McGill, uses an optimization algorithm and a dynamic electron collimator located at the end of the electron applicator to define a field size and electron energy. This treatment technique could be incorporated into MMCTP for routine use. Other desirable features include: handling of 4D imaging data-sets, multi-modality imaging and fusion, digital radiograph reconstruction, gamma function comparison tool, dose reconstruction from patient portal images. 


\section{References}

[1] Central axis depth dose data for use in Radiotherapy. British Journal of Radiology, 25.

[2] Prescribing, recording, and reporting photon beam therapy. ICRU 50, International Commission on Radiation Unite and Measurements, 1993.

[3] A Ahnesjö, M Saxner, and A Trepp. A pencil beam model for photon dose calculation. Med. Phys., 19:263-273, 1992.

[4] K S AL-Yahya. Implementation of Monte Carlo Treatment Planning for Lung Cancer Patients. Master's thesis, McGill University, Montreal, Quebec, May 2002.

[5] H F Batho. Lung corrections in cobalt 60 beam therapy. J. Can. Assoc. Radiol., 15:79, 1964.

[6] M J Berger. Methods in computational physics, volume 1. Academic Press, New York, 44 edition, 1963.

[7] M J Berger and S M Seltzer. Tables of energy losses and ranges of electrons and positrons. SP 3012, NASA, 1964.

[8] A F Bielajew. Lecture notes: Photon monte carlo simulation. NRCC Report PIRS 0393, Ionizing Radiaion Standards, 2000.

[9] A F Bielajew, H Hirayama, W R Nelson, and D W O Rogers. History, overview and recent improvements of EGS4. NRCC Report NRC-PIRS 0436, Ionizing Radiaion Standards.

[10] A Brahme. Dosimetric precision requirements in radiation therapy. Acta. Radiol. Oncol., 23:379-391, 1984.

[11] I J Chetty and J P Seuntjens. Monte Carlo-based Clinical Treatment Planning: Issues for Consideration. presented at AAPM summer school Windsor, Ontario, June 2006. 
[12] J R Clarkson. A note on depth doses in fields of irregular shape. British Journal of Radiology, 14:256, 1941.

[13] J Dai. Validation of monte carlo techniques for 3D-CRT of lung cancer patients and comparative evaluation of treatment plans. Master's thesis, McGill University, Montreal, Quebec, May 2005.

[14] J O Deasy, A I Blanco, and V H Clark. CERR: A computational environment for radiotherapy research. Med. Phys., 30:979, 2003.

[15] R Doucet, J P Seuntjens, M Olivares, F DeBlois, and E B Podgoršak. Experimental verification of Monte Carlo calculated dose distributions in heterogeneous phantoms in electron beams. In Proceedings of the 47 th annual scientific meeting, Kelowna, June 2001. Canadian Organization of Medical Physicists.

[16] J V Dyk. The Modern Technology of Radiation Oncology, volume 1. Medical Physics Publishing, Madison, Wisconsin, 1st edition, 1999.

[17] I Kawrakow. VMC++, Electron and Photon Monte Carlo Calculations Optimized for Radiation Treatment Planning. In Proceedings of the Monte Carlo 2000 Conference, Lisbon.

[18] I Kawrakow. Improved modeling of multiple scattering in the Voxel Monte Carlo model. Med. Phys., 24:505-517, 1997.

[19] I Kawrakow and D W O Rogers. The EGSnrc Code System: Monte Carlo Simulation of Electron and Photon Transport. NRCC Report PIRS, Ionizing Radiaion Standards.

[20] J Lehmann, C H Siantar, D E Wessol, C A Wemple, D Nigg, J Cogliati, T Daly, M Descalle, T Flickinger, D Pletcher, and G DeNardo. Monte Carlo treatment planning for molecular target radiotherapy within the MINERVA system. Phys. Med. Biol., 50:947-958, 2005.

[21] H H Liu, T R Mackie, and E C McCullough. Modeling photon output caused by backscattered radiation into the monitor chamber from collimator jaws using a Monte Carlo technique. Med. Phys., 27:737-744, 200.

[22] D A Low and J F Dempsey. Evaluation of the gamma dose distribution comparison method. Med. Phys., 30:2455-2464, 2003. 
[23] D A Low, W B Harms, S Mutic, and J A Purdy. A technique for the quantitative evaluation of dose distributions. Med. Phys., 25:656-661, 1998.

[24] C M Ma, E Mok, A Kapur, T Pawlicki, D Findley, S Brain, and K Forster. Clinical implementation of a Monte Carlo treatment planning system. Med. Phys., 26:2133, 1999.

[25] C I Mark. Image-guided radiotherapy using 2D and 3D ultrasound combined with Monte Carlo dose calculations in prostate treatments. Master's thesis, McGill University, Montreal, Quebec, December 2005.

[26] W R Nelson, H Hirayama, and D W Rogers. The EGS4 Code system. SLAC 256, Stanford Linear Accelerator Center Report Stanford CA, 1985.

[27] D W O Rogers. Ionizing radiation dosimetry and medical physics. NRCC Report 51, Ionizing Radiaion Standards, 1995.

[28] D W O Rogers, A F Bielajew, T R Mackie, and S S Kubsad. The OMEGA Project: treatment planning for electron-beam radiotherapy using Monte Carlo techniques. Phys. Med. Biol., 35:285, 1990.

[29] D W O Rogers, B A Faddegon, G X Ding, C M Ma, J We, and T R Mackie. BEAM: A Monte Carlo code to simulate radiotherapy treatment units. Med. Phys., 22:503-524, 1995.

[30] D W O Rogers, B Walters, and I Kawrakow. BEAMnrc Users Manual. Ionizing Radiation Standards National Research Council of Canada NRCC Report PIRS0509, Ottawa, Ontario, November 2005.

[31] E B Podgoršak. Review of Radiation Oncology Physics : A Handbook for Teachers and Students, volume 1. IAEA, Vienna, Austria, 1st edition, 2004.

[32] F Verhaegen and J Seuntjens. Monte Carlo modelling of external radiotherapy photon beams. Phys. Med. Biol., 48:107-164, 2003.

[33] F Verhaegen and J Seuntjens. International Workshop on Current Topics in Monte Carlo Treatment Planning. Phys. Med. Biol., 50:20, 2005.

[34] F Verhaegen, R Symonds-Tayler, H Liu, and A E Nahum. Backscatter towards the monitor ion chamber in high-energy photon and electron beams charge integration versus Monte Carlo simulation. Phys. Med. Biol., 45:3159-3170, 2000. 
[35] B R B Walters, I. Kawrakow, and D W O Rogers. History by history statistical estimators in the BEAM code system. Med. Phys., 29:2745, 2002. 